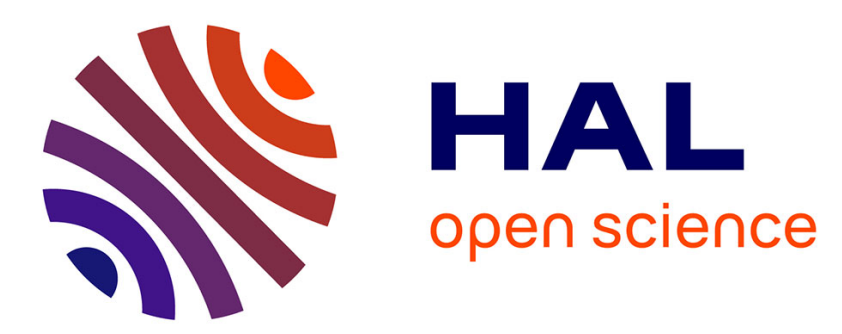

\title{
Review on the Brownian Dynamics Simulation of Bead-Rod-Spring Models Encountered in Computational Rheology
}

C Cruz, Francisco Chinesta, Gilles Regnier

\section{- To cite this version:}

C Cruz, Francisco Chinesta, Gilles Regnier. Review on the Brownian Dynamics Simulation of BeadRod-Spring Models Encountered in Computational Rheology. Archives of Computational Methods in Engineering, 2012, 19, pp.227-259. 10.1007/s11831-012-9072-2 . hal-01197328

\section{HAL Id: hal-01197328 \\ https://hal.science/hal-01197328}

Submitted on 11 Sep 2015

HAL is a multi-disciplinary open access archive for the deposit and dissemination of scientific research documents, whether they are published or not. The documents may come from teaching and research institutions in France or abroad, or from public or private research centers.
L'archive ouverte pluridisciplinaire HAL, est destinée au dépôt et à la diffusion de documents scientifiques de niveau recherche, publiés ou non, émanant des établissements d'enseignement et de recherche français ou étrangers, des laboratoires publics ou privés. 


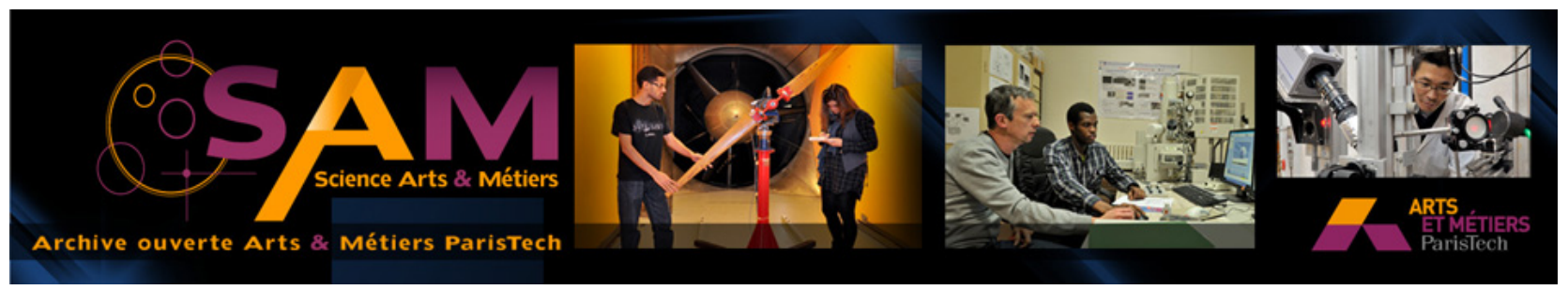

\section{Science Arts \& Métiers (SAM)}

is an open access repository that collects the work of Arts et Métiers ParisTech researchers and makes it freely available over the web where possible.

This is an author-deposited version published in: http://sam.ensam.eu

Handle ID: .http://hdl.handle.net/10985/9991

\section{To cite this version :}

C CRUZ, F CHINESTA, Gilles REGNIER - Review on the Brownian Dynamics Simulation of Bead-Rod-Spring Models Encountered in Computational Rheology - Archives of Computational Methods in Engineering - Vol. 19, p.227-259 - 2012 


\title{
Review on the Brownian Dynamics Simulation of Bead-Rod-Spring Models encountered in Computational Rheology
}

\author{
C. Cruz · F. Chinesta - G. Régnier
}

\begin{abstract}
Kinetic theory is a mathematical framework intended to relate directly the most relevant characteristics of the molecular structure to the rheological behaviour of the bulk system. In other words, kinetic theory is a micro-to-macro approach for solving the flow of complex fluids that circumvents the use of closure relations and offers a better physical description of the phenomena involved in the flow processes. Cornerstone models in kinetic theory employ beads, rods and springs for mimicking the molecular structure of the complex fluid. The generalized bead-rod-spring chain includes the most basic models in kinetic theory: the freely jointed bead-spring chain and the freely-jointed bead-rod chain. Configuration of simple coarse-grained models can be represented by an equivalent FokkerPlanck (FP) diffusion equation, which describes the evolution of the configuration distribution function in the physical and configurational spaces. FP equation can be a complex mathematical object, given its multidimensionality, and solving it explicitly can become a
\end{abstract}

C. Cruz

Laboratoire des Procédés et Ingénierie en Mécanique et Matériaux (PIMM, UMR CNRS 8006), Arts et Métiers ParisTech, 151 bd de l'Hôpital, 75013 Paris, France

F. Chinesta

Corporate Foundation International Chair, Ecole Centrale de Nantes, 1 rue de la Noë - BP 92101, 44321 Nantes Cédex 3, Nantes, France

\section{G. Régnier}

Laboratoire des Procédés et Ingénierie en Mécanique et Matériaux (PIMM, UMR CNRS 8006), Arts et Métiers ParisTech, 151 bd de l'Hôpital, 75013 Paris, France

Tel.: +33-144246413

Fax: +33-144246290

E-mail: gilles.regnier@ensam.eu difficult task. Even more, in some cases, obtaining an equivalent FP equation is not possible given the complexity of the coarse-grained molecular model. Brownian dynamics can be employed as an alternative extensive numerical method for approaching the configuration distribution function of a given kinetic-theory model that avoid obtaining and/or resolving explicitly an equivalent $\mathrm{FP}$ equation. The validity of this discrete approach is based on the mathematical equivalence between a continuous diffusion equation and a stochastic differential equation as demonstrated by Itô in the 1940s. This paper presents a review of the fundamental issues in the $\mathrm{BD}$ simulation of the linear viscoelastic behaviour of bead-rod-spring coarse grained models in dilute solution. In the first part of this work, the BD numerical technique is introduced. An overview of the mathematical framework of the BD and a review of the scope of applications are presented. Subsequently, the links between the rheology of complex fluids, the kinetic theory and the BD technique are established at the light of the stochastic nature of the bead-rod-spring models. Finally, the pertinence of the present state-ofthe-art review is explained in terms of the increasing interest for the stochastic micro-to-macro approaches for solving complex fluids problems. In the second part of this paper, a detailed description of the BD algorithm used for simulating a small-amplitude oscillatory deformation test is given. Dynamic properties are employed throughout this work to characterise the linear viscoelastic behaviour of bead-rod-spring models in dilute solution. In the third and fourth part of this article, an extensive discussion about the main issues of a BD simulation in linear viscoelasticity of diluted suspensions is tackled at the light of the classical multibead-spring chain model and the multi-bead-rod chain model, respectively. Kinematic formulations, integra- 
tion schemes and expressions to calculate the stress tensor are revised for several classical models: Rouse and Zimm theories in the case of multi-bead-spring chains, and Kramers chain and semi-flexible filaments in the case of multi-bead-rod chains. The implemented BD technique is, on the one hand, validated in front of the analytical or exact numerical solutions known of the equivalent FP equations for those classic kinetic theory models; and, on the other hand, is control-set thanks to the analysis of the main numerical issues involved in a BD simulation. Finally, the review paper is closed by some concluding remarks.

Keywords Brownian dynamics - Kinetic theory · Linear viscoelasticity

\section{Notes on Notation}

$\begin{array}{ll}\text { Scalars } & \text { Lightface } \\ \text { Vectors and Tensors } & \text { Boldface } \\ (\mathbf{A})^{i j} & \text { Component } i j \text { of tensor } \mathbf{A} \\ (\mathbf{A})_{t} & \text { Tensor or vector } \mathbf{A} \text { at instant } t\end{array}$

\section{Introduction}

This paper presents a state-of-the-art review of the numerical methods in Brownian dynamics used to model the rheological behaviour of bead-rod-spring models. Generalized bead-rod-spring models are coarse-grained representations of the molecular structure of complex fluids. In addition, bead-rod-spring models are the cornerstone of the kinetic theory; a formal framework that try to explain the flow dynamics of non-Newtonian fluids by relating the molecular structure with the bulk flow behaviour. In order to reveal the intrinsic dynamic response of those coarse-grained models this article focuses on the linear viscoelastic behaviour of their dilute solutions, where inter-molecular interactions are neglected.

This introductory section is decomposed in three parts. In the first one, a general view of the Brownian dynamics framework and its scope of applications are presented. In the second section, a brief outline about the rheology of complex fluids followed by its relation with the kinetic theory is presented. The inherent stochastic nature of the coarse-grained models is revealed establishing a connection with the Brownian dynamics technique. Finally, in the third section a line of argument is developed to show the interest of using the stochastic approach when solving kinetic theory problems.

\subsection{Brownian Dynamics Modelling}

A mathematical representation of all physical phenomena occurring in the physical world cannot be treated efficiently using only one kind of model. In general, the choice of a given modelling approach depends on the time and length scales involved in the phenomenon. For example, quantum mechanics is a suitable model for representing the physical phenomena occurring at the level of quantum particles and atomic sub-particles, but probably the quantum mechanics framework is not the more convenient choice to model a tensile relaxation test in a polyethylene film. Today, quantum mechanics is the finest description of the physical behaviour of matter. Going up in length and time scales of modelling requires different mathematical formulations with the aim of encapsulate the phenomena occurring at finer scales. For example, between the molecular and macroscopic scales, models must contain a mathematical architecture taking into account the rapid oscillations occurring at lower scales (i.e. atoms, smaller molecules or smaller particles). Brownian dynamics (BD) is precisely one of the mathematical frameworks employed for representing the physics at the micro-meso scale (1nm $10 \mu \mathrm{m})$. In BD the rapid thermal oscillations at lower scales are tackled as a stochastic variable [1].

The historical motivation that inspired the development the Brownian dynamics framework was the publication of the Robert Brown's observations about the random motion of pollen particles in water. The irregular path described by those pollen particles was explained afterwards as the result of random thermal collisions between pollen particles and water molecules. By the way, a mathematic formalism describing the motion of a particle submitted to stochastic forces was introduced by the French physicist Paul Langevin [2]. In fact, a general differential stochastic equation is also known as a Langevin equation. Considering a Brownian particle (in the large sense, i.e. a discrete portion of matter submitted to stochastic forces coming from the surrounding homogeneous media) of mass $M$, the instantaneous general Langevin equation of motion writes:

$M \ddot{\mathbf{r}}=-\nabla U(\mathbf{r})-\varsigma M \dot{\mathbf{r}}+\varsigma M \boldsymbol{\kappa}(\mathbf{r})+\mathbf{F}^{(b)}$

where $\mathbf{r}$ is the particle position, $\varsigma$ is the specific friction coefficient, $\boldsymbol{\kappa}$ is the homogenous velocity gradient coming from the suspension at the position of the particle, $U$ is the sum of all the particle interaction potentials (e.g. mechanical, electrostatic, magnetic) and $\mathbf{F}^{(b)}$ is the Brownian force acting on the particle. This last variable confers to the differential equation its stochastic nature. Keeping in mind that $\mathbf{F}^{(b)}$ is originated from independent thermal collisions with the surrounding particles; 
the central limit theorem conduct intuitively to think that $\mathbf{F}^{(b)}$ behaves following a Gaussian process. A formal definition of $\mathbf{F}^{(b)}$ in coherence with the stochastic calculus framework writes as follows:

$\mathbf{F}_{t}^{(b)}=\sqrt{2 k_{B} T \varsigma M} \frac{d(\mathbf{W})_{t}}{d t}$

where $k_{B}$ is the Boltzman's constant, $T$ is the absolute temperature and $(\mathbf{W})_{t}$ is a Wiener process at instant $t$. $(\mathbf{W})_{t}$ is a well-defined Gaussian process itself, hence can be defined by the first and second moments of its distribution:

$$
\begin{aligned}
& \left\langle(\mathbf{W})_{t}\right\rangle=\mathbf{0} \\
& \left\langle(\mathbf{W})_{t_{1}} \otimes(\mathbf{W})_{t_{2}}\right\rangle=\min \left(t_{1}, t_{2}\right) \boldsymbol{\delta}
\end{aligned}
$$

where $\langle\ldots\rangle$ represents an ensemble average and $\delta$ is the unit tensor. In a BD simulation, we are rather interested in the numerical implementation of a multi-dimensional increment of a Wiener process $\Delta \mathbf{W}_{t t^{\prime}}=(\mathbf{W})_{t}-(\mathbf{W})_{t^{\prime}}$. Using the central limit theorem, it can be demonstrated that those increments are independent and follow also a Gaussian distribution with the next moments:

$$
\begin{aligned}
& \left\langle(\mathbf{W})_{t}-(\mathbf{W})_{t^{\prime}}\right\rangle=\mathbf{0} \\
& \left\langle\left((\mathbf{W})_{t}-(\mathbf{W})_{t^{\prime}}\right) \otimes\left((\mathbf{W})_{t}-(\mathbf{W})_{t^{\prime}}\right)\right\rangle=\left|t-t^{\prime}\right| \boldsymbol{\delta}
\end{aligned}
$$

When implementing an explicit integration of a $\mathcal{D}$ dimensional Wiener process (defined in an orthogonal basis) in the time interval $\left[0, t_{n}\right]$, equi-partitioned in $n$ intervals of size $\Delta t=t_{i}-t_{i-1}$, it is just necessary to define the initial value of the Wiener process, $(\mathbf{W})_{0}=\mathbf{0}$, and calculate iteratively the increments as follows: $(\mathbf{W})_{t_{i}}=(\mathbf{W})_{t_{i-1}}+(\Delta \mathbf{W})_{t_{i} t_{i-1}}$, where each component of $\Delta \mathbf{W}_{t_{i} t_{i-1}}$ is obtained from an independent one-dimension normal distribution $\mathrm{N}(0, \Delta t)$.

Definition of the stochastic Brownian force given in (2) is coherent with the principle of equi-partition of energy, the fluctuation-dissipation theorem (in the sense that frictional force depends only on the instantaneous local velocity) and the formalisms of stochastic calculus [3].

Brownian dynamics is a limit case of the Langevin dynamics framework. In BD the inertial effects are neglected; in other words, BD supposes that no average acceleration takes place on the Brownian particle. In some physical sciences Brownian dynamics is also known as overdamped Langevin dynamics. Non-inertia assumption is justified in the insignificance of the inertial forces coming from small-mass particles in front of the viscous and thermal forces acting on the same particle. Brownian dynamics equation of motion for a Brownian particle writes therefore as follows:

$$
\begin{aligned}
\mathbf{0}= & -\nabla U(\mathbf{r})-\varsigma M \dot{\mathbf{r}}+\varsigma M \boldsymbol{\kappa}(\mathbf{r})+ \\
& \sqrt{2 k_{B} T \varsigma M} \frac{d(\mathbf{W})_{t}}{d t}
\end{aligned}
$$

Defining $\zeta=M \varsigma$ as the friction coefficient and $D=$ $k_{B} T / \zeta$ as the diffusion coefficient of the particle, the instantaneous velocity of the Brownian particle can be written as follows:

$\dot{\mathbf{r}}=\frac{-\nabla U(\mathbf{r})}{\zeta}+\boldsymbol{\kappa}(\mathbf{r})+\sqrt{2 D} \frac{d(\mathbf{W})_{t}}{d t}$

Brownian dynamics simulations have been used intensively to study the physics of different kind of macromolecules and soft matter systems [4], as for example, the rheological behaviour of polymer $[5,6,7,8,9,10]$, the dynamics of proteins and DNA $[11,12,13,14]$, the flow behaviour of colloids $[15,16,17,18,19,20,21]$, the structural dynamics of liquid-crystals $[22,23]$ and the dynamics of carbon nanotubes $[24,25,26,27]$.

\subsection{Rheology of Complex Fluids and Kinetic Theory}

A comprehensive theoretical modelling of the problems in fluid dynamics requires a suitable formulation of the constitutive equation for the momentum flux or stress tensor. Flow of complex fluids differs from that one occurring in classical Newtonian fluids because it cannot be described by using a simple viscous constitutive equation. In fact, the mechanical response of a complex fluid to a given deformation appears to be viscoelastic, in other words, the complex fluid exhibits a mechanical behaviour intermediate between perfect elastic solid and a perfect viscous liquid. Two viscoelastic regimes can be identified in function of the imposed strain. If the viscoelastic response is independent of the applied strain then we assist to the linear viscoelastic behaviour. This mechanical regime is typically associated with very small deformations. On the other hand, when the viscoelastic response depends on the applied strain, then we fall in the non-linear viscoelastic regime. This paper is particularly focused on linear viscoelasticity because this mechanical regime constitutes the natural first approach in a rheological modelling framework.

The main aim of rheology consists to establish a suitable constitutive equation that relates stress and strain tensors for a given fluid and, sometimes, for a particular kind of flow. Kinetic theory in complex fluids is an exhaustive mathematical framework that looks for explaining the bulk flow phenomena based on the molecular structure of the fluid system. In that sense, 
kinetic theory is one of the tools employed by rheologists for generating suitable constitutive equations for non-Newtonian fluids. Formal framework in kinetic theory is built on coarse-grained representations of the molecular structure involved in the flow phenomena. Historically, the former coarse-grained molecular models appeared to emulate the polymer molecular structure. That explains why frequently the literature makes reference to polymer kinetic theory.

The coarse-grained molecular models in kinetic theory constitute a first attempt to relate the molecular structure to the bulk flow mechanics. Basic coarsegrained models emulate the most relevant characteristics of the molecular structure using beads, rods and springs as constitutive blocks. In what follows, an overview about the origins of bead-rod and the bead-spring models is illustrated based on the descriptions made by Bird et al elsewhere [28].

\subsubsection{Bead-Rod Model}

Bead-rod models were proposed to emulate the structure of linear polymer chains. The first natural representation consisted in neglecting the pendant atoms or groups linked to the central chain and replacing this central chain by a series of beads and mass-less rods, where beads represent the constitutive atoms of the central chain and rods represent the chemical bonds between them. At this level of representation, the stochastic nature of the coarse-grained model emerges because all possible thermal interactions with the solvent molecules are reduced to an instantaneous stochastic force acting on each bead. Adjacent bonds in a linear polymer chain are restricted to very narrow intervals of solid angle values due to sterical hindering [29]. Based on this physical argument bead-rod chain model with fixed solid angles and restricted bond-rotation was proposed.

A simpler model considers that the rotational hindrances can be neglected, but the solid angles between adjacent rods are maintained. This representation is known as the freely rotating chain model. A complete series of articles about the non-equilibrium dynamics of the freely rotating chain model has been published before [30].

An even coarse-grained bead-rod model neglect any rotating and bending hindering between adjacent rods. This model is known as the freely jointed multi-bead-rod chain or, simply, Kramers chain. In this case, beads do not represent central chain atoms, but a portion of the polymer chain, typically 10-20 monomer units. In short, a Kramers chain can be described as an ensemble of $n$ beads linked by $n-1$ rods of length $a$ (see Fig. 1), where each bead is characterised by a drag coefficient $\zeta$.

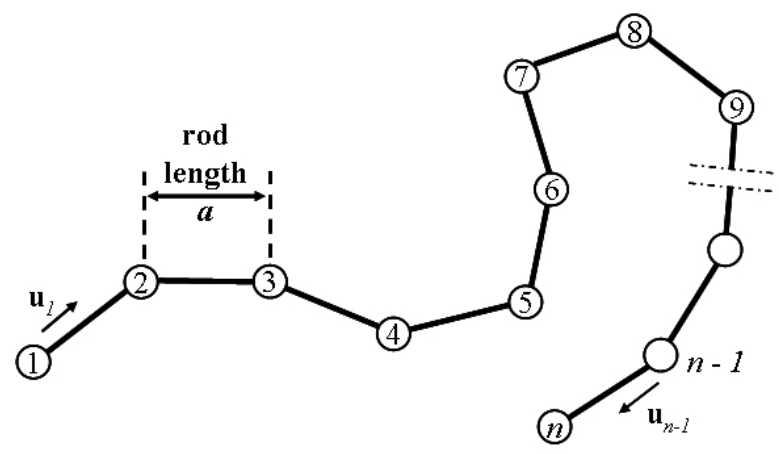

Fig. 1 Freely-jointed multi-bead-rod model composed of $n$ beads and $n-1$ rods of length $a$

It has been demonstrated that supposing a randomwalk distribution for the Kramers chain configuration the mean-square end-to-end distance of the chain at equilibrium writes (see for example [31]):

$\left\langle r^{2}\right\rangle_{e q}=a^{2}(n-1)$

On the other hand, using a thermodynamic approach, it was shown that the average tension in a Kramers chain suspended in a solvent bath at temperature $T$ and extended to a fixed end-to-end vector $\mathbf{r}$ (whose norm does not exceed one half of the contour length of the bead-rod chain) writes as follows [28]:

$\mathbf{F}^{(c)}(\mathbf{r})=\frac{3 k_{B} T}{a^{2}(n-1)} \mathbf{r}$

It is important to point out that previous expression is only exact when supposing a random-walk distribution for the configuration distribution function of the constitutive rods. In fact, this condition is only true for a large number of beads $n$ [28]. It means that a 3-dimensional freely-jointed multi-bead-rod model behaves mechanically as a Hookean spring of null natural length with a spring constant $H$ equals to:

$H=\frac{3 k_{B} T}{a^{2}(n-1)}=\frac{3 k_{B} T}{\left\langle r^{2}\right\rangle_{e q}}$

\subsubsection{Bead-Spring Model}

Based on the characteristics of the mechanical behaviour of a freely-jointed multi-bead-rod chain, it seemed natural to propose another up-level of coarse-graining for representing the structure of linear polymer chains. Actually, a linear polymer chain can be modelled as a series of springs, where each spring represents several hundreds of central chain atoms. Springs are linked by beads that concentrate masses and friction effects associated to the replaced central chain atoms. This model is called the freely-jointed multi-bead-spring chain (see 
Fig. 2). In principle, constitutive springs have null natural length, but simulations with non-zero natural length springs (e.g. Fraenkel-type) are also frequently employed. Several disadvantages appear at this level of coarsegraining; for instance, contour length is no more constant and, furthermore, if the spring potential is supposed Hookean, the chain can be infinitely extended, something that is physically unrealistic.

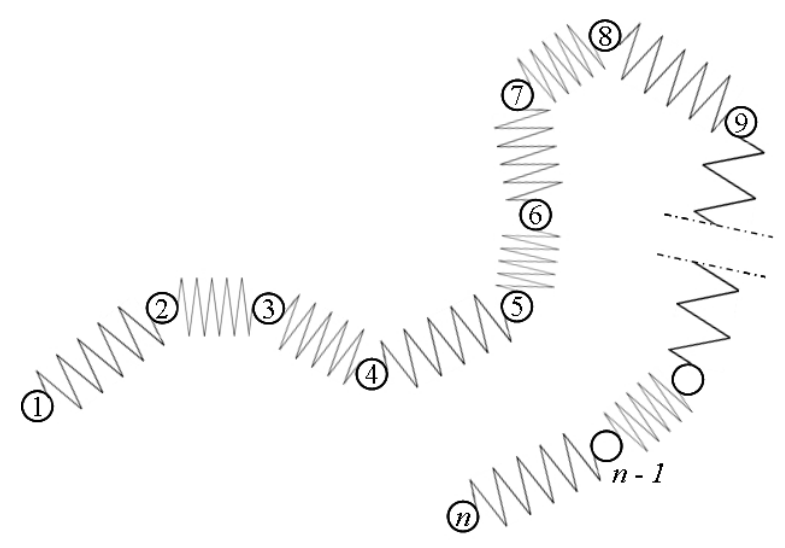

Fig. 2 Freely-jointed multi-bead-spring model composed of $n$ beads and $n-1$ springs $(T>0 \mathrm{~K})$

Considering a freely-jointed multi-bead-spring chain constituted of $n$ beads linked by $n-1$ springs, it results not surprising that the average end-to-end distance at equilibrium be an extension of the analogue expression derived for the freely-jointed multi-bead-rod model:

$\left\langle r^{2}\right\rangle_{e q}=\frac{3 k_{B} T(n-1)}{H}$

In spite of the multi-bead-rod-spring models were initially developed in the framework of the polymer kinetic theory, those coarse-grained representations has been extended to model a vast kind of systems, including proteins, DNA, virus and carbon nanotubes. As mentioned before, this article will be focused on the BD simulation of the linear viscoelastic behaviour of the bead-rod-spring models in absence of inter-molecular interactions (hypothesis of infinitely diluted solution).

One can consider two different approaches when tackling with kinetic theory models in micro-to-macro simulations: a deterministic and a stochastic one. On the one hand, the deterministic approach deals with the direct solving of the FP equation at the expense of that the FP equation can be effectively formulated. FP or diffusion equation is a deterministic partial differential equation describing the evolution of the probability density function (in configurational space) of a given coarse-grained model. On the other hand, the stochastic approach proposes an extensive analysis of a representative population of coarse-grained models and the estimation of macroscopic properties by properly averaging the physical state of the population under consideration. Stochastic approach is conceivable for all coarse-grained kinetic theory models, even for those that do not have an equivalent FP equation. This paper presents the main issues of the latter approach by using a BD simulation tool for predicting the dynamic behaviour of bead-rod-spring models in solution.

\subsection{The Interest of the Stochastic Approach}

A natural question that appears when dealing with flows of complex fluids is why to prefer using an extensive (and probably costly) method for integrating the FP diffusion equation of a given molecular model. An immediate answer to such question is simply that in some cases a FP diffusion equation is not available given the complexity of the coarse-grained model and, hence, the stochastic approach is the only method for accessing to the dynamic behaviour of the molecular model.

On the other hand, in the classical framework of the kinetic theory, the equations of motion for the different constitutive blocks in a given coarse-grained model (beads, rods, springs) added to the continuity equation for the configurational distribution function can be used to build a FP diffusion equation. The common utility of this deterministic diffusion equation consists in recover the exact physical response of the system when submitted to a given external flow field by integrating the $\mathrm{FP}$ equation in the configurational space and in time (if looking for dynamic properties) in order to develop constitutive equations. Normally, those constitutive equations are employed a posteriori to calculate more complex flows by using a continuum mechanics framework. The crucial point in the previously described process lies on the mathematical method employed for integrating the diffusion equation given its multidimensional character. FP equation is a multidimensional function that depends on time and all the space coordinates chosen to define the configuration of the coarse-grained molecular model. It is necessary to point out that there is a vast collection of numerical strategies developed to solve this problematic integration $[32,33,34,35,36,37$, $38,39,40,41,42,43,44,45,46,47,48,49]$.

However, it appears that the stochastic approach for solving the dynamics of coarse-grained models (even for those that have an equivalent FP diffusion equation) is employed more and more. In this case, we deal directly with the stochastic differential equation of motion for 
each coarse-grained system. In order to evaluate a given physical property, an average over a finite population of model systems has to be performed (in analogy to the integration of an equivalent FP equation in the configurational space). The main difficulties emerging with this approach are the associated stochastic noise, the low convergence order and the computational cost required to overcome the first two mentioned issues. Nevertheless, the growing-up leaning for tackling with the stochastic approach is due mainly to four reasons: 1) the dealing with complex coarse-grained structural descriptions that have not an equivalent FP formulation, 2) the enhanced possibility of enrich a given coarsegrained model by analyzing its configurational evolution under designed flow conditions, 3) the raising capacities of the computational tools that reduce simulation times, and 4) the interesting idea of resolving viscoelastic flow engineering problems without resorting to closed-forms of the constitutive equations for the stress tensor. This last idea means to establish microto-macro simulations where the configuration state of a population of coarsed-grained systems lets to calculate the stress field, allowing to compute the evolution of the flow field and, subsequently, to update the configurational space of coarse-grained models by solving the stochastic differential equations of motion $[50,51$, $52,53,54,55,56]$.

Given the actual interest in the stochastic approach for solving the micro-to-macro modelling of viscoelastic flows, it is pertinent to review the fundamental numerical aspects associated with the solution of the differential stochastic equations for the simplest models in the kinetic theory framework. In that way, this paper analyses the main issues about the implementation of BD simulations for the bead-spring and bead-rod models. BD simulations in this paper are focused on predicting the dynamic response of dilute solutions of those coarse-grained kinetic theory models in the framework of linear viscoelasticity.

\section{A Brownian Dynamics Algorithm for Simulating Linear Viscoelastic Behaviour}

From now on, an extensive Brownian dynamics modelling is systematically used to predict the dynamic response of a given bead-rod-spring chain model highly diluted within a Newtonian solvent. A classical rheological test used in linear viscoelasticity is simulated: a dynamic test of small-amplitude oscillatory deformation. Suspension is supposed confined between two parallel infinite-plates in the case of 3D simulations (see Fig. 3). In the case of $2 \mathrm{D}$ simulations, bead-rod-spring chain models are supposed confined in a plane $x z$ of Fig. 3.

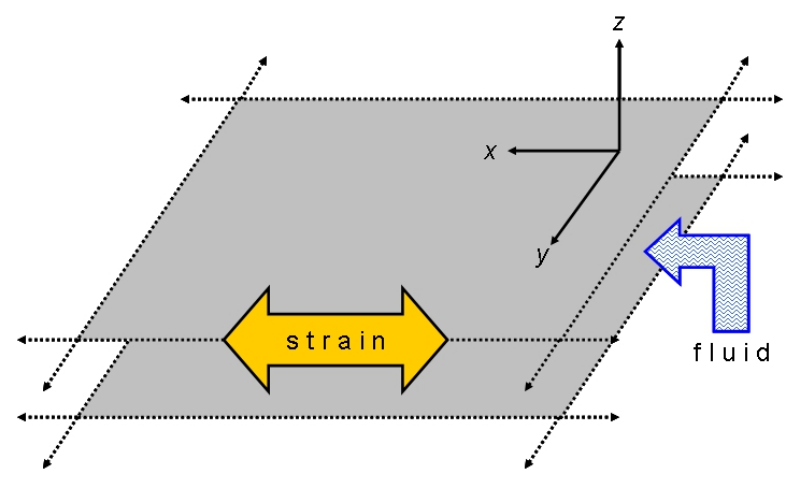

Fig. 3 Parallel infinite plates where BD simulations in shear rheology are carried out. Upper plate slides in relation to the lower one along the $x$ axis for homogeneously shearing the fluid confined in between

Oscillatory strain function is imposed by the relative sliding movement of the plates (or bars); inducing a homogeneous deformation within the suspension confined between the plates (or bars). Rheological response is supposed coming mainly from the bulk of the suspension, therefore wall and free-surface effects are ignored. Rheological properties are calculated from a properly defined average over $N$ chain models, where $N$ is the number of bead-rod-spring elements in the stochastic simulation. By the way, number of freedom degrees in the Brownian dynamics simulation is proportional to $N$. As suspension is supposed highly diluted, no interaction effects are considered between chain models and, hence, no computational storage of the relative positions between chains is necessary. A general flowchart of the algorithm intended to simulate a frequency sweep test in linear regime is presented in Fig. 4.

The first step consists to obtain a configuration in thermal equilibrium. No external flow is imposed. Configuration of the BD system ( $N$ bead-rod-spring models) evolves in time under the action of the forces coming from the friction with the solvent, the internal energy potentials and the thermal interaction with the solvent molecules (Brownian effect). Time integration in this equilibrium stage is carried out until at least one of two criteria is satisfied: stabilization of the stored internal energy (when it can be estimated) or integration during at least three times the longest characteristic time associated with an equivalent bead-rod-spring chain whose FP equation has an analytical solution. For instance, as internal energy is not accessible for freelyjointed bead-rod models, equilibrium step is carried out 


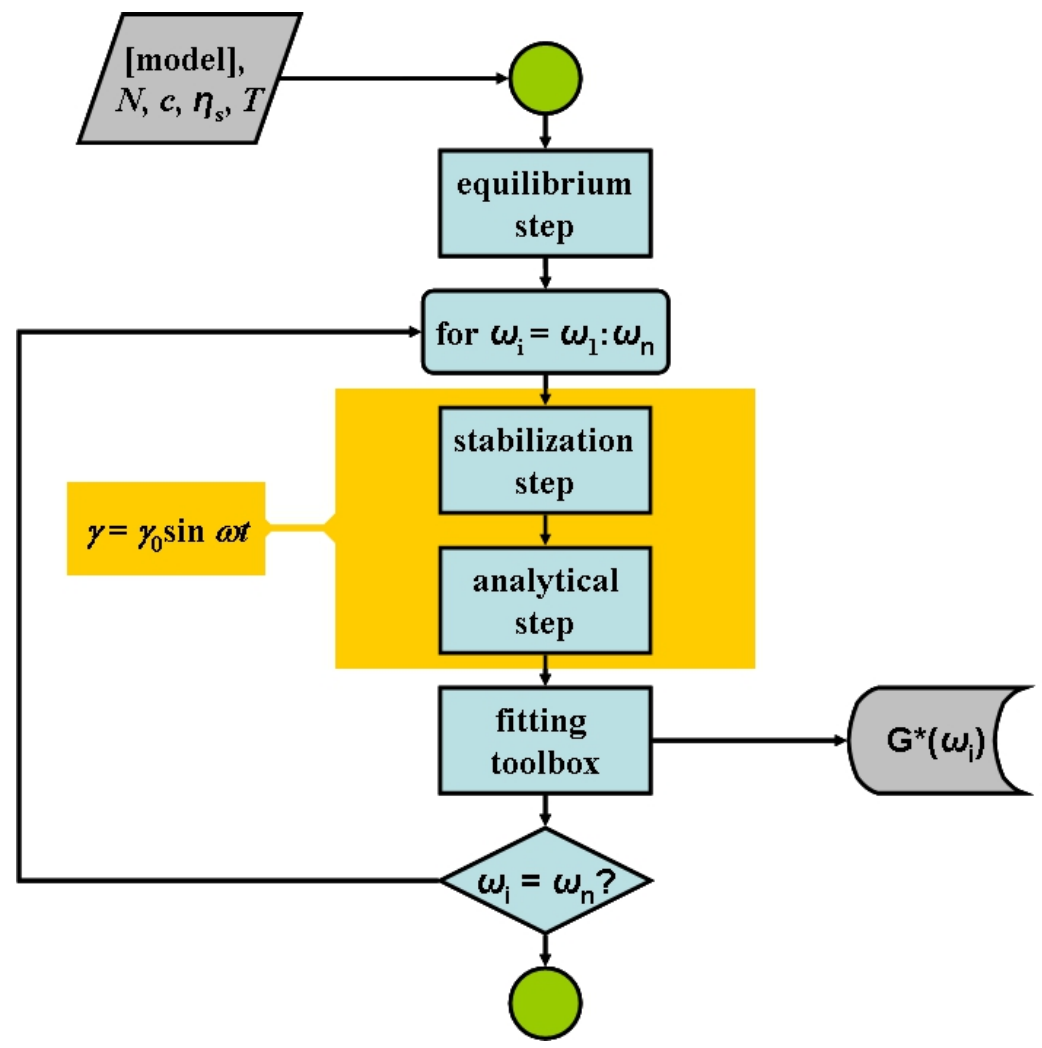

Fig. 4 Flowchart of the general BD algorithm for simulating a small-amplitude oscillatory deformation test during three times the rotational time of an equivalent multi-bead rigid-rod with the same length.

Once the equilibrium configuration of the BD system has been obtained, the dynamic strain function $\gamma=\gamma_{0} \sin \omega t$ can be applied, where $\gamma_{0}$ is a deformation within the linear regime. Frequency sweep test is carried out on a finite number of frequencies homogeneously distributed in logarithmic scale between the lowest frequency $\omega_{1}$ and the highest frequency $\omega_{n}$. The $\mathrm{BD}$ prediction of the complex modulus at each tested frequency is composed of two steps. First, a dynamic stabilisation of the BD system is carried out either until internal energy dynamic stabilization or during at least three times the longest characteristic time of an equivalent bead-rod-spring model before starting the second stage, called analytical step, in which the BDcalculated shear-stress signal is stored during one-anda-half periodic oscillations. This signal is used as entry variable in a fitting toolbox with the aim to determine the parameters $\left(\tau_{0}, \delta\right)$ of a smooth shear-stress function $\tau=\tau_{0} \sin (\omega t+\delta)$ by using a Newton-based errorminimization methodology. Dynamic complex modulus at each frequency is then computed easily in the next way:

$G^{*}(\omega)=G^{\prime}(\omega)+i G^{\prime \prime}(\omega)=\frac{\tau_{0}}{\gamma_{0}} \cos \delta+i \frac{\tau_{0}}{\gamma_{0}} \sin \delta$

where $G^{\prime}$ is the storage modulus and $G^{\prime \prime}$ is the loss modulus.

\section{BD of Generalized Bead-Spring Models}

Even though the multi-bead-rod model appeared chronologically earlier than the multi-bead-spring model, this latter is tackled preliminarily here because it offers a simpler mathematical structure that is reflected immediately on the simplicity of the BD numerical methods associated with. In addition, due probably to the simpler mathematic formalisms, the multi-spring-bead model has been more recurrently employed to model the dynamics of complex fluids.

\subsection{Kinematic and Dynamic Formulation}

Let consider the multi-bead-spring chain model in Fig. 2. In what follows, a multi-bead-spring chain is constituted of $n$ beads joined by $n-1$ non-bendable springs. The instantaneous position of each bead is defined in a coordinate reference system by the vector $\mathbf{r}_{i}$. An alternative way for describing the position and the orientation of the multi-bead-spring chain is possible defining the centre of masses of the chain, $\mathbf{r}_{c}$ and the connector vector $\mathbf{Q}_{i}$ between consecutive beads:

$\mathbf{r}_{c}=\frac{1}{n} \sum_{i=1}^{n} \mathbf{r}_{i}$

$\mathbf{Q}_{i}=\mathbf{r}_{i+1}-\mathbf{r}_{i}$ 
Multi-bead-spring chain is supposed suspended into a solvent at temperature $T$. Solvent is considered a Newtonian fluid with viscosity $\eta_{s}$. Concentration of the multi-bead-spring chain is defined in terms of the density of chains, $c$ chains per volume unit. As the solution is supposed highly diluted no interaction between multibead-spring chains is considered. Moreover, flow field in the overall suspension is supposed homogeneous, in the meaning that the rate-of-strain tensor is the same in all points of the flow field or, at least, in the scale of twice the contour length of the multi-bead-spring chain [28].

In the kinematic formulation of this kind of coarsegrained model is presumed that all changes of momentum are concentrated on beads. Furthermore, an assumption of inertia-less is employed; due to the insignificance of inertial forces (small masses) with respect to friction and thermal forces acting on them. In what follows a detailed description of the forces acting on beads is presented.

- Hydrodynamic drag force acting on bead $i, \mathbf{F}_{i}^{(h)}$ This force describes the resistance experienced by the bead as it moves through the fluid. One of the simplest ways to express this force is given by the Stoke's law, which considers the hydrodynamic drag force proportional to the difference between the fluid velocity at the bead position and the averaged bead velocity. A more general expression takes into account a hydrodynamic interaction component that comes from the physical perturbation of the local flow field due to the global chain movement. Hydrodynamic drag force acting on bead $i$ writes as follows:

$\mathbf{F}_{i}^{(h)}=\boldsymbol{\zeta}\left(\boldsymbol{\kappa}\left(\mathbf{r}_{i}\right) \cdot \mathbf{r}_{i}+\mathbf{v}_{i}^{\prime}-\dot{\mathbf{r}}_{i}\right)$

where $\boldsymbol{\zeta}$ is a second-order friction tensor, $\boldsymbol{\kappa}$ is the gradient of the bulk velocity field and $\dot{\mathbf{r}}_{i}$ is the averaged instantaneous bead velocity. On the other hand, $\mathbf{v}_{i}^{\prime}$ accounts for the variation in the local flow field around $\mathbf{r}_{i}$ due to the motion of the other beads in the same chain.

In polymer kinetic theory, Rouse model neglects this intra-molecular interaction (i.e. $\mathbf{v}_{i}^{\prime}=\mathbf{0}$ ); resorting to the well-known free draining motion hypothesis [57]. On the other hand, theories taking into account the hydrodynamic interaction suppose that the bead velocity $\mathbf{v}_{i}^{\prime}$ depends linearly on the hydrodynamic forces acting on the others beads inside the chain:

$\mathbf{v}_{i}^{\prime}=-\sum_{j} \boldsymbol{\Omega}_{i j} \cdot \mathbf{F}_{j}^{(h)}$

where $\boldsymbol{\Omega}_{i j}$ is the Oseen-Burgers hydrodynamic interaction tensor associated with a given pair of beads $i$ and $j$. Say that the perturbation of a velocity field in a given point of the space depends linearly on the hydrodynamic forces acting in the surroundings of that point presupposes a Maxwellian velocity distribution [28]. Oseen-Burgers hydrodynamic tensor writes as follows:

$\boldsymbol{\Omega}_{i j}=\frac{1}{8 \pi \eta_{s} r_{j i}}\left(\boldsymbol{\delta}+\frac{\mathbf{r}_{j i} \otimes \mathbf{r}_{j i}}{r_{j i}^{2}}\right)$ for $i \neq j$

where $\mathbf{r}_{j i}=\mathbf{r}_{i}-\mathbf{r}_{j}$ and $r_{j i}=\left\|\mathbf{r}_{j i}\right\|$. On the other hand, $\boldsymbol{\Omega}_{i i}=\mathbf{0}$. Zimm model, in the context of polymer kinetic theory, takes into account the hydrodynamic interaction effect using the equilibriumaveraged Oseen-Burgers tensor [58]:

$\left\langle\boldsymbol{\Omega}_{i j}\right\rangle_{e q}=\frac{1-\delta_{i j}}{6 \pi \eta_{s}}\left\langle\frac{1}{r_{j i}}\right\rangle_{e q} \boldsymbol{\delta}$ for $i \neq j$

where $\delta_{i j}$ is the Kronecker delta.

- Intra-molecular force acting on bead $i, \mathbf{F}_{i}^{(\phi)}$

This force corresponds to the sum of all the spring forces acting on bead $i$. In a multi-bead-spring chain, total intra-molecular force on bead $i$ writes as follows:

$\mathbf{F}_{i}^{(\phi)}=\sum_{j=1}^{n-1}\left(\delta_{i j}-\delta_{i j+1}\right) \mathbf{F}_{j}^{(c)}$

where $\mathbf{F}_{j}^{(c)}=\partial \phi_{j} / \partial \mathbf{Q}_{j}$ is the connector force acting on bead $j$ along spring $j$, defined as the gradient of the spring potential energy $\phi_{j}$. Linear force law is the simplest connector force:

$$
\begin{aligned}
\mathbf{F}_{i}^{(H)} & =\frac{\partial \phi_{i}^{(\mathrm{LIN})}}{\partial \mathbf{Q}_{i}} \\
& =\frac{\partial}{\partial \mathbf{Q}_{i}}\left(\frac{1}{2} H\left(\sqrt{\mathbf{Q}_{i} \cdot \mathbf{Q}_{i}}\right)^{2}\right) \\
& =H \mathbf{Q}_{i}
\end{aligned}
$$

Previous Hookean-type law force is based on the entropic analysis over a tighten random-walk polymer chain. $H$ is a Hookean spring constant that can be related to temperature and some molecular structural parameters (see $\$ 1.2 .1)$. The linear law force is only valid for large polymer chains and small strain regimes. Hence, Hookean law force is inadequate for processes involving large deformations. This fact motivated the use of non-linear force laws, for instance:

- Finite extensible non-linear elastic (FENE) force law [59]

$$
\mathbf{F}_{i}^{(\mathrm{FENE})}=\frac{H \mathbf{Q}_{i}}{1-\left(Q_{i} / Q_{i, 0}\right)^{2}}
$$

where $Q_{i}=\left\|\mathbf{Q}_{i}\right\|$ and $Q_{i, 0}$ is the maximal extension of the spring. 
- Inverse Langevin (IL) force law [60]

$\mathbf{F}_{i}^{(\mathrm{IL})}=\frac{k_{B} T}{a} \mathcal{L}^{-1}\left[\frac{\mathbf{Q}_{i}}{Q_{i, 0}}\right]$

where $a$ is typically twice the persistence length $l_{p}$ and the Langevin function $\mathcal{L}$ is given by $\mathcal{L}(x)=$ $\operatorname{coth}(x)-x^{-1}$. Persistence length in a polymer molecule is a measure of the flexibility of the chain; in other terms, the direction of the chainaxis in a quiescent polymer molecule is uncorrelated only along contour length distances equal or higher than $2 l_{p}$.

- Worm-like chain (WLC) force law [61]

$$
\mathbf{F}_{i}^{(\mathrm{WLC})}=\frac{k_{B} T}{a}\left[\begin{array}{c}
\frac{1}{2} \frac{1}{\left(1-Q_{i} / Q_{i, 0}\right)^{2}} \\
-\frac{1}{2}+\frac{2 Q_{i}}{Q_{i, 0}}
\end{array}\right] \frac{\mathbf{Q}_{i}}{Q_{i, 0}}
$$

A comparison of the various spring-force laws is presented in Fig. 5. Observe that FENE-like force laws are linear at small extensions and tends to infinite when extension approaches the finite maximal length.

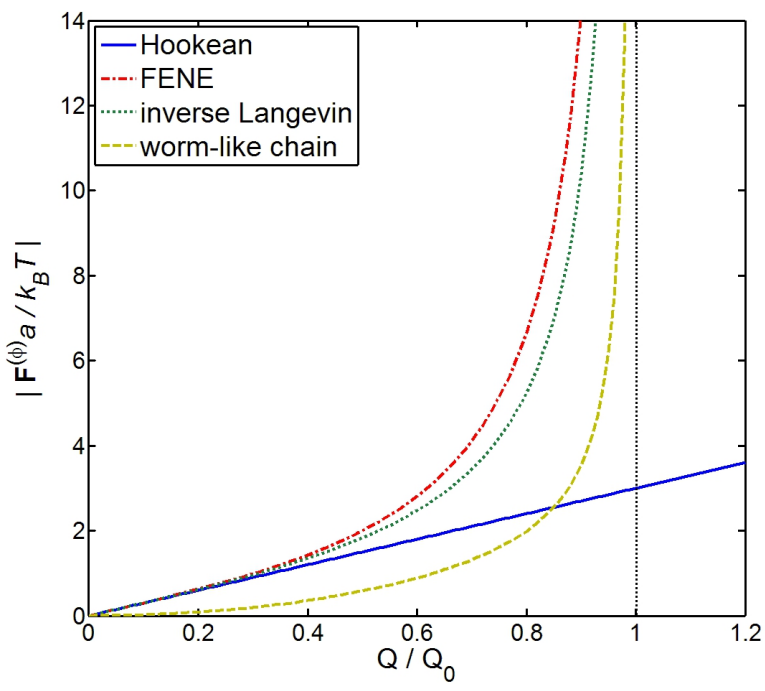

Fig. 5 Curves of the spring connector force $\left|\mathbf{F}^{(\phi)}\right|$ in function of the reduced spring extension for several spring force laws employed in polymer kinetic theory

- Brownian force acting on bead $i, \mathbf{F}_{i}^{(b)}$

The Brownian force accounts for the change of momentum at each bead (supposed as Brownian particles) due to the ensemble of instantaneous collisions of the solvent molecules against it. In nature, those collisions are faster than the bead motion, that is why Brownian forces are considered as stochastic variables in the $\mathrm{BD}$ time scale. Due to the isotropic condition of those collisions, Brownian force is treated mathematically as a quantity with zero mean in time and space (ergodicity principle).
On the other hand, the second-moment of the distribution of Brownian forces must equilibrate the dissipative forces [3]. In short, an "instantaneous" Brownian force distribution in a BD simulation is characterised as a Gaussian process with the next first and second moments:

$$
\begin{aligned}
& \left\langle\mathbf{F}_{i}^{(b)}(t)\right\rangle=\mathbf{0} \\
& \left\langle\mathbf{F}_{i}^{(b)}(t) \otimes \mathbf{F}_{j}^{(b)}(t+\Delta t)\right\rangle=\frac{2 k_{B} T}{\Delta t} \boldsymbol{\zeta}
\end{aligned}
$$

where $\Delta t$ is a discrete approximation of a differential in time. It is important to mention that in an orthogonal basis of $\mathcal{D}$ dimensions the multi-dimensional Gaussian process can be decoupled in $\mathcal{D}$ independent uni-dimensional Gaussian processes [3].

- External forces acting on bead $i, \mathbf{F}_{i}^{(e)}$

Gravitational and electromagnetic fields can induce non-negligible external forces over the beads of the system. In what follows these forces are not considered.

Taking into account that beads are considered as Brownian particles, the inertial effects are neglected. In such context, the force balance must to be satisfied at each time and at each bead $i$ :

$\mathbf{F}_{i}^{(h)}+\mathbf{F}_{i}^{(\phi)}+\mathbf{F}_{i}^{(b)}+\mathbf{F}_{i}^{(e)}=\mathbf{0}$

Using the definition of the hydrodynamic drag force, previous differential stochastic equation can be transformed to explicit the bead velocity:

$$
\begin{gathered}
\frac{d \mathbf{r}_{i}}{d t}=\mathbf{v}_{i}^{\prime}+\left[\boldsymbol{\kappa}\left(\mathbf{r}_{i}\right) \cdot \mathbf{r}_{i}\right]+\boldsymbol{\zeta}^{-1} \mathbf{F}_{i}^{(\phi)}+ \\
\boldsymbol{\zeta}^{-1} \mathbf{F}_{i}^{(b)}+\boldsymbol{\zeta}^{-1} \mathbf{F}_{i}^{(e)}
\end{gathered}
$$

Integration in time of previous stochastic equation governs the kinematic evolution of the bead-spring system. As any numerical method, in a BD simulation time is treated as a discrete variable and, hence, different numerical integration schemes can be proposed.

\subsection{Integration Schemes}

Given the structure of Eq. (28) a simple explicit integration scheme inspired by the numerical resolution of ordinary differential equations appears as a natural integration scheme. In fact, the Euler-Maruyama method is the simplest way to integrate numerically Eq. (28). Given an initial configuration at time $t,\left(\mathbf{r}_{i}\right)_{t}$, it is possible to estimate $\left(\mathbf{r}_{i}\right)_{t+\Delta t}$ by using the next formula: 


$$
\begin{aligned}
\left(\mathbf{r}_{i}\right)_{t+\Delta t}= & \left(\mathbf{r}_{i}\right)_{t}+\left(\mathbf{v}_{i}^{\prime}+\left[\boldsymbol{\kappa}\left(\mathbf{r}_{i}\right) \cdot \mathbf{r}_{i}\right]+\boldsymbol{\zeta}^{-1} \mathbf{F}_{i}^{(\phi)}\right)_{t} \Delta t \\
& +\left(\sqrt{2 k_{B} T \boldsymbol{\zeta}^{-1}}\right) \Delta \mathbf{W}_{i}
\end{aligned}
$$

where $\Delta \mathbf{W}$ is a random increment following a multidimensional Wiener process. This random Wiener process follows a normal distribution with the next first and second moments:

$\left\langle\Delta \mathbf{W}_{i}\right\rangle=\mathbf{0}$

$$
\left\langle\left(\Delta \mathbf{W}_{i}\right)_{t} \otimes\left(\Delta \mathbf{W}_{j}\right)_{t+\Delta t}\right\rangle=\Delta t \boldsymbol{\delta}
$$

Rewriting Eq. (29) only in terms of the time step $\Delta t$ we have:

$$
\begin{aligned}
\left(\mathbf{r}_{i}\right)_{t+\Delta t}= & \left(\mathbf{r}_{i}\right)_{t}+\left(\mathbf{v}_{i}^{\prime}+\left[\boldsymbol{\kappa}\left(\mathbf{r}_{i}\right) \cdot \mathbf{r}_{i}\right]+\boldsymbol{\zeta}^{-1} \mathbf{F}_{i}^{(\phi)}\right)_{t} \Delta t \\
& +\left(\sqrt{2 k_{B} T \boldsymbol{\zeta}^{-1} \Delta t}\right) \Delta \mathbf{N}_{i}(0,1)
\end{aligned}
$$

where $\Delta \mathbf{N}_{i}(0,1)$ is a multi-dimensional Gaussian increment of mean 0 and variance 1 . Performance of the Euler-Maruyama method is extremely sensitive to the time step employed. In fact, convergence of the integration scheme is only guaranteed when $\Delta t \rightarrow 0$ [62]. Suppose a stochastic differential equation with the next general form:

$d X=A(X) d t+B(X) d W$

An integration scheme converges strongly with order $v$ at time $t_{\max }$ if the next condition is satisfied:

$\left\langle\left|X\left(t_{\max }\right)-X_{\text {true }}\left(t_{\max }\right)\right|^{2}\right\rangle^{1 / 2} \leq C(\Delta t)^{v}$

for all time step lengths equal or inferior to $\Delta t$ and $C$ being a positive constant. It has been proven that the Euler-Maruyama method exhibit a low order of strong convergence $v=1 / 2$ [3]. Identification of the maximal time step satisfying the strong convergence criteria for a specific $C$ value is normally done by trial and error and plotting the error in function of the time step. In spite of the low order of convergence, when the drift and diffusive coefficients $(A(X)$ and $B(X)$ in Eq. (33) respectively) are almost constants, the Euler-Maruyama method is the most frequently used integration scheme. Thus, this explicit integration scheme is widely used in BD simulations for bead-spring systems with Hookeansprings.

Now, when the drift and diffusion coefficients become more complex, the fully explicit method is not satisfying and high order integration schemes are required. Such is the case when non-linear spring potentials are employed to guarantee a finite spring extensibility. In fact, the main difficulty appearing with the numerical temporal integration of this kind of beadspring systems is that the bead displacements have to be bounded in order to not exceed the maximal spring extension. For describing those high-order integration algorithms is better to express the bead-spring chain dynamics (given in the Eq. (28)) in terms of the connector vector $\mathbf{Q}_{i}$ :

$$
\begin{aligned}
\frac{d \mathbf{Q}_{i}}{d t}= & \left(\mathbf{v}_{i+1}^{\prime}-\mathbf{v}_{i}^{\prime}\right)+\left[\boldsymbol{\kappa}\left(\mathbf{r}_{i}\right) \cdot \mathbf{Q}_{i}\right]+\boldsymbol{\zeta}^{-1}\left(\mathbf{F}_{i+1}^{(\phi)}-\mathbf{F}_{i}^{(\phi)}\right) \\
& +\boldsymbol{\zeta}^{-1}\left(\mathbf{F}_{i+1}^{(b)}-\mathbf{F}_{i}^{(b)}\right)
\end{aligned}
$$

A first approximation to solve the previous equation containing non-linear spring forces consists in use a conventional explicit scheme (Euler-Maruyama method) and just reject the updating step that produces a not-physical displacement (maximal spring extension exceeded). Some practical rejection criteria and useful advices for the choice of the time step are given elsewhere [3].

More appropriate methods to integrate Eq. (35) are based on implicit schemes. For example, Somasi et al. [63] proposed a fully implicit scheme inspired in the two-step semi-implicit algorithm proposed by Öttinger for FENE dumbbells [3]. In general, at each time step, those implicit methods are composed of a predictor step followed by a corrector one. Given a bead-spring configuration at the time $t,\left(\mathbf{Q}_{i}\right)_{t}$, the configuration after one time step is generated as follows:

- Predictor step. Using a conventional explicit scheme a test configuration $\mathbf{Q}_{i}^{*}$ is calculated as follows:

$$
\mathbf{Q}_{i}^{*}=\left(\mathbf{Q}_{i}\right)_{t}+\left[\begin{array}{l}
\left(\mathbf{v}_{i+1}^{\prime}-\mathbf{v}_{i}^{\prime}\right)_{t} \\
+\left[\boldsymbol{\kappa}\left(\mathbf{r}_{i}\right) \cdot\left(\mathbf{Q}_{i}\right)_{t}\right] \\
+\boldsymbol{\zeta}^{-1}\left(\mathbf{F}_{i+1}^{(\phi)}-\mathbf{F}_{i}^{(\phi)}\right)_{t} \\
+\boldsymbol{\zeta}^{-1}\left(\mathbf{F}_{i+1}^{(b)}-\mathbf{F}_{i}^{(b)}\right)_{t}
\end{array}\right] \Delta t
$$

- Corrector step. Rewriting the spring potential forces in terms of the connector forces (Eq. (20)) and using the test configuration obtained in the predictor step to average the flow-field contribution to the drag forces is possible to write a corrector step as follows:

$$
\begin{aligned}
& \overline{\mathbf{Q}}_{i}+2 \boldsymbol{\zeta}^{-1} \overline{\mathbf{F}}_{i}^{(c)} \Delta t=\left(\mathbf{Q}_{i}\right)_{t} \\
& +\frac{1}{2}\left[\boldsymbol{\kappa}\left(\mathbf{r}_{i}\right) \cdot\left(\mathbf{Q}_{i}\right)_{t}+\boldsymbol{\kappa}\left(\mathbf{r}_{i}\right) \cdot \mathbf{Q}_{i}^{*}\right] \Delta t \\
& +\boldsymbol{\zeta}^{-1}\left(\overline{\mathbf{F}}_{i-1}^{(c)}+\left(\mathbf{F}_{i+1}^{(c)}\right)_{t}\right) \Delta t \\
& +\boldsymbol{\zeta}^{-1}\left(\mathbf{F}_{i+1}^{(b)}-\mathbf{F}_{i}^{(b)}\right)_{t} \Delta t
\end{aligned}
$$


where spring forces for connectors $i$ and $i-1$ are treated implicitly when solving for $\overline{\mathbf{Q}}_{i}$. Rearreangement of Eq. (37) generates a cubic equation for the magnitude of $\overline{\mathbf{Q}}_{i}$, whose unique root is inferior to the maximal spring extensibility. In the final stage of the corrector step, spring force of the connector $i+1$ is given by Eq. (37) and spring forces for connectors $i$ and $i-1$ are tackled implicitly again:

$$
\begin{aligned}
& \overline{\mathbf{Q}}_{i}^{[n]}+2 \boldsymbol{\zeta}^{-1}\left(\overline{\mathbf{F}}_{i}^{(c)}\right)^{[n]} \Delta t=\left(\mathbf{Q}_{i}\right)_{t} \\
& +\frac{1}{2}\left[\boldsymbol{\kappa}\left(\mathbf{r}_{i}\right) \cdot\left(\mathbf{Q}_{i}\right)_{t}+\boldsymbol{\kappa}\left(\mathbf{r}_{i}\right) \cdot \overline{\mathbf{Q}}_{i}^{[n-1]}\right] \Delta t \\
& +\boldsymbol{\zeta}^{-1}\left(\left(\overline{\mathbf{F}}_{i-1}^{(c)}\right)^{[n]}+\mathbf{F}_{i+1}^{(c)}\right) \Delta t \\
& +\boldsymbol{\zeta}^{-1}\left(\mathbf{F}_{i+1}^{(b)}-\mathbf{F}_{i}^{(b)}\right)_{t} \Delta t
\end{aligned}
$$

Previous equation gives also a cubic equation for the $n$th approximation of $\overline{\mathbf{Q}}_{i}^{[n]}$ and, hence, an iterative process can be formulated (doing $\overline{\mathbf{Q}}_{i}^{[n-1]}$ equal to $\left.\overline{\mathbf{Q}}_{i}^{[n]}\right)$ until difference between consecutive solutions is inferior to a specified tolerance $\varepsilon$ :

$$
\sqrt{\sum_{i=1}^{n-1}\left(\overline{\mathbf{Q}}_{i}^{[n]}-\overline{\mathbf{Q}}_{i}^{[n-1]}\right)^{2}} \leq \varepsilon
$$

Once the iterative process converges, $\overline{\mathbf{Q}}_{i}^{[n]}$ is said to be the spring configuration at time $t+\Delta t,\left(\mathbf{Q}_{i}\right)_{t+\Delta t}$, with the guarantee that the lengths of the connector vectors are allowed physical ones.

In spite of the heavier calculus involved at each time step in relation to the explicit algorithm, the advantage of the implicit schemes is that larger time steps can be employed. For such reason, each modelling requires a particular analysis for determining which integration method produces a more efficient BD simulation.

Several studies about the numerical integration of non-linear stochastic equations and time step width control are found elsewhere, as well as, relevant examples of BD simulations of bead-spring systems with finite-extensibility spring potentials $[64,65,66]$.

\subsection{Stress Tensor Calculation}

In order to obtain the rheological behaviour of any coarse-grained molecular model it is necessary to extract the stress tensor information from its mechanical balance at each instant. Total shear-stress tensor $\tau$ in a suspension is supposed to be the sum of the contribution coming from the solvent $\boldsymbol{\tau}_{s}$ and another one coming from the suspended entities $\boldsymbol{\tau}_{p}$ (in this case, the multi-bead-spring chains) [28]:

$\tau=\tau_{s}+\tau_{p}$
It is important to notice that at equilibrium (i.e. no external forces and no external-flow field), the total shear stress tensor $\boldsymbol{\tau}$ is zero. Assuming that the solvent is a Newtonian fluid, then previous Eq. (40) can be rewritten as follows:

$\boldsymbol{\tau}=\eta_{s} \dot{\gamma}+\tau_{p}$

where $\eta_{s}$ is the solvent viscosity and $\dot{\gamma}$ is the homogeneous rate-of-strain tensor. Anyway, to model the intrinsic rheological response of the bead-spring chain, one is particularly interested on the shear stress contribution coming from the suspended particles $\boldsymbol{\tau}_{p}$. In the early literature of polymer kinetic theory there are several formal derivations of expressions accounting for the shear stress tensor contribution coming from multibead-spring chains suspended in liquid media $[67,68]$. According to those developments in a bead-spring chain there are three principal physical effects contributing to the total shear stress tensor: 1) the intra-molecular forces across the connector vectors; 2) the external forces acting on beads and 3) the transport of momentum caused by the displacement of the beads. Those are not the only sources contributing to the physical mechanical state of the system, but in the framework of a standard rheological test those described mechanisms are definitively the most important.

Based on this deduction guideline, the Kramers expression for the shear-stress tensor contribution coming from a multi-bead-spring chain writes as follows:

$$
\begin{aligned}
\boldsymbol{\tau}_{p}= & c \sum_{k=1}^{n-1}\left\langle\mathbf{Q}_{k} \otimes \mathbf{F}_{k}^{(c)}\right\rangle-c \sum_{v=1}^{n} \sum_{k=1}^{n-1} B_{v k}\left\langle\mathbf{Q}_{k} \otimes \mathbf{F}_{v}^{(e)}\right\rangle \\
& -(n-1) c k_{B} T \boldsymbol{\delta}
\end{aligned}
$$

where $B_{v k}$ is a scalar operator associated with bead $v$ and spring $k$ given by [28]:

$B_{v k}=\left\{\begin{array}{cl}\frac{k}{n} & k<n \\ -\left[1-\frac{k}{n}\right] & k \geq n\end{array}\right.$

In fact, the configuration of a bead-spring chain can be specified alternatively by the position of the centre of mass of the chain $\mathbf{r}_{c}$ and the $n-1$ connector vectors $\mathbf{Q}_{k}$ as follows:

$\mathbf{r}_{v}=\mathbf{r}_{c}+\sum_{k=1}^{n-1} B_{v k} \mathbf{Q}_{k}$

In the right side of Kramers expression in Eq. (42) are easily identifiable the three mentioned physical effects contributing to the shear-stress tensor: the first term corresponds to the intra-molecular forces contribution, the second one represents the contribution of the external forces and the third one accounts for the 
momentum transfer of beads (Brownian contribution). This last term is an isotropic contribution to the shear stress tensor based on the supposition of a Maxwellian velocity distribution.

Kramers expression can be slightly transformed by writing the spring-bead chain conformation in terms of the distance of each bead to the centre of mass of the chain, $\mathbf{R}_{v}=\mathbf{r}_{v}-\mathbf{r}_{c}$, and by using the expression relating the intra-molecular force acting on bead $v, \mathbf{F}_{v}^{(\phi)}$, with the connector force $\mathbf{F}_{k}^{(c)}$ associated to spring $k$ (Eq. 20). Such form is known as the modified-Kramers expression for the shear-stress tensor contribution of a bead-spring chain:

$$
\begin{aligned}
\boldsymbol{\tau}_{p}= & -c \sum_{v=1}^{n}\left\langle\mathbf{R}_{v} \otimes\left(\mathbf{F}_{v}^{(\phi)}+\mathbf{F}_{v}^{(e)}\right)\right\rangle \\
& -(n-1) c k_{B} T \boldsymbol{\delta}
\end{aligned}
$$

Now, combining previous equation with the bead force balance in a multi-bead-spring chain given in Eq. (27) and neglecting any external force a much simpler expression for the shear stress tensor can be obtained:

$\boldsymbol{\tau}_{p}=c \sum_{v=1}^{n}\left\langle\mathbf{R}_{v} \otimes \mathbf{F}_{v}^{(h)}\right\rangle$

where the result,

$\sum_{v=1}^{n}\left\langle\mathbf{R}_{v} \otimes \mathbf{F}_{v}^{(b)}\right\rangle=(n-1) k_{B} T \boldsymbol{\delta}$

has been employed supposing again a Maxwellian velocity distribution. Shear-stress tensor contribution in Eq. (46) is known as the Kramers-Kirkwood expression $[67,30]$.

Non intuitively, BD simulations with bead-spring models in $2 \mathrm{D}$ and $3 \mathrm{D}$ produced the same results. In what follows of the current section 3, only the predictions in $3 \mathrm{D}$ are given.

\subsection{The Rouse Model}

Rouse model is a polymer kinetic theory model that mimics the structure of a linear polymer chain using a multi-bead-spring chain, where intra-molecular interactions are neglected. Springs in the Rouse model follows a Hookean law, based on the results of the end-toend entropic tension experimented by a freely-jointed Kramers chain (following a Gaussian distribution configuration) suspended in an isothermal solvent bath (see $\S 1.2 .1)$. No external forces are considered. A formal compendium of the theory can be found in the recognized publication of Rouse in 1953 [57].

\subsubsection{The Hookean-Dumbbell}

The simplest version of the Rouse model is the (twobeads)-(one-spring) system or, better known as, the elastic-dumbbell model. It has been found that the mechanical response of the dumbbell model is equivalent to that one of a Maxwell element (spring-dashpot in series) used by rheologists to model a general linear viscoelastic behaviour [69].

Given the simplicity of the dumbbell model, it will be used to study the main numerical issues of the BD simulations for bead-spring models. An explicit integration scheme has been employed in a three-dimensional BD simulation. In what follows, the equilibrium properties and dynamical behaviour are analysed in detail.

Equilibrium Properties A (two-beads)-(Hookean-spring) system at thermal equilibrium and in absence of an external-flow field has an average square end-to-end distance $\left\langle r^{2}\right\rangle_{e q}$ equal to:

$\left\langle r^{2}\right\rangle_{e q}=\frac{3 k_{B} T}{H}$

An important aspect in BD simulations is the number of entities (or trajectories) required to converge to the central values with satisfactory statistics. In Fig. 6 the BD performance to estimate the square end-toend distance in function of the population considered is presented.

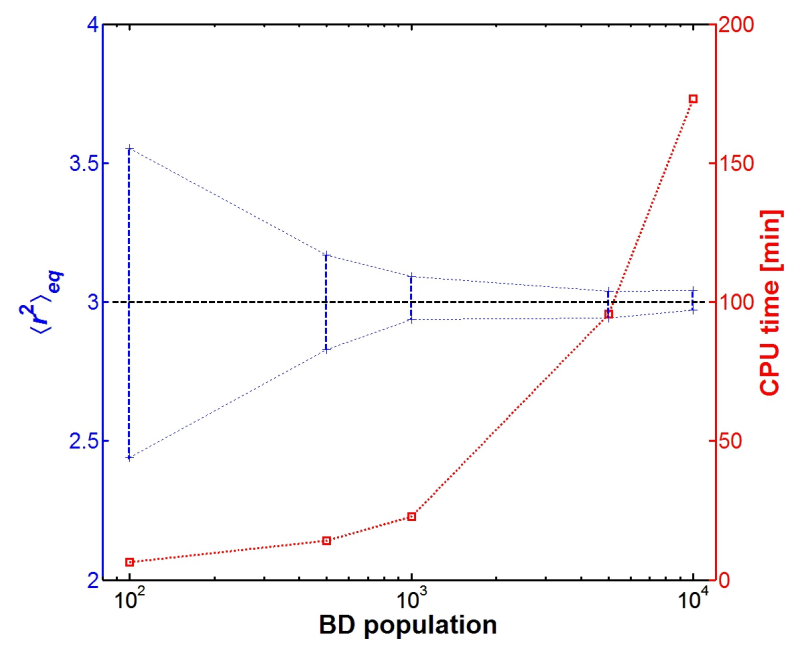

Fig. 6 Statistics of the square end-to-end distance of a Hookean dumbbell in thermal equilibrium (during 1500 time steps) in function of the BD population. $H, \zeta$ and $c k_{B} T$ are fixed to 1 in a consistent system of units. Square end-to-end distance at equilibrium is equal to 3 according to the kinetic theory. A time step equal to $\lambda_{H} / 250$ has been employed

The simulated Hookean dumbbell has a square endto-end distance at equilibrium equal to 3 (given by 
Eq. 48) and a relaxation time of $\lambda_{H}=0.25$ according to the kinetic theory framework. A short time step ( $\left.\Delta t=\lambda_{H} / 250\right)$ was employed in the simulation in order to marginalize the effect of the integration scheme convergence. BD performance is measured as the dispersion of the average square end-to-end distance (95\% of confidence) for 1500 time steps once the system has reached an equilibrium configuration (i.e. after at least 3 times the relaxation time). A reduction of the relative dispersion with respect to the central value is observed from $18.5 \%$ for a population of $10^{2}$ dumbbells to $1.1 \%$ for a population of $10^{4}$ dumbbells. As expected, a linear evolution of the computational time (Intel@) Core $^{\mathrm{TM}} \mathrm{T} 73002.00 \mathrm{GHz}$ ) in function of the number of dumbbells simulated is noticed. For this particular case, a good compromise between simulation accuracy and computational time is obtained for populations between of $10^{3}$ and $5 \times 10^{3}$ dumbbells.

Another important issue in $\mathrm{BD}$ simulations, especially when employing an explicit integration scheme, is time step. In Fig. 7 the influence of the time step on the convergence of the integration scheme is revealed. In this case a population of $10^{4}$ dumbbells is employed with the aim to attenuate the noise coming from the random number generation of statistical distributions and isolate the effects originated by the integration scheme.

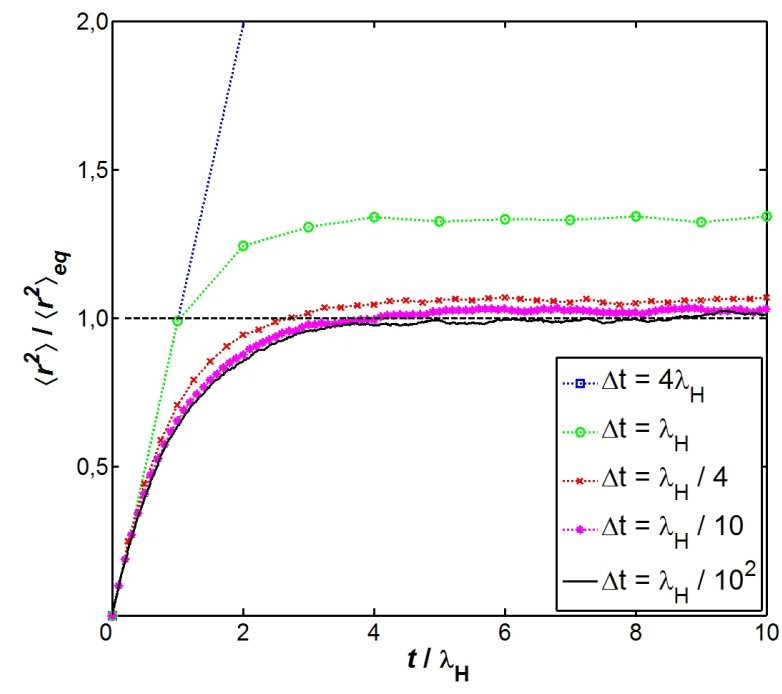

Fig. 7 Evolution of the reduced square end-to-end distance of a dumbbell after sudden thermal activation for different time steps in the BD simulation. $H, \zeta$ and $c k_{B} T$ are fixed to 1 in a consistent system of units. Square end-to-end distance is made dimensionless using the equilibrium value given by the kinetic theory. A population of $10^{4}$ dumbbells has been employed

In the Fig. 7 the evolution of the squared end-to-end distance of a Hookean dumbbell, after a sudden ther- mal activation, is presented for different time steps. In absence of temperature, the end-to-end distance of a dumbbell is null. That is the reason why all curves begin at the origin. According to the kinetic theory, a time equal to four times the relaxation time is enough for a Hookean dumbbell model to reach the equilibrium endto-end distance at constant temperature. From Fig. 7, a complete divergence of the integration scheme is observed when a time step bigger than the main relaxation time is employed. For a time step equal to the relaxation time and equal to a quarter of the relaxation time integration scheme does not diverge, but the steady values are inaccurate regarding the exact solution. On the other hand, a finer convergence towards the central values is observed as the time step gets smaller than a tenth of the relaxation time.

At thermal equilibrium the shear-stress tensor is zero, so for a Hookean dumbbell Kramers expression (Eq. (42)) writes:

$\boldsymbol{\tau}_{p, e q}=c\left\langle\mathbf{Q} \otimes \mathbf{F}^{(c)}\right\rangle-c k_{B} T \boldsymbol{\delta}=\mathbf{0}$

which could be written also as:

$$
\frac{\left\langle\mathbf{Q} \otimes \mathbf{F}^{(c)}\right\rangle_{e q}}{k_{B} T}=\boldsymbol{\delta}
$$

Using a time step equal to $\lambda_{H} / 100$ and an equilibrated population of $10^{3}$ dumbbells (i.e. a BD system equilibrated during a time equal to three times the main relaxation time), the simulation produces a stable shear stress tensor. Third of the trace of the tensor in the left member of Eq. (50) has a mean value equal to $0.99 \pm 0.03$ (95\% confidence) during 600 times steps. On the other hand, the out-of-diagonal components of the same tensor have an absolute mean value equal to $0.005 \pm 0.063$ (95\% confidence) during 600 iterations.

Dynamic Properties The linear viscoelastic behaviour of a Hookean-dumbbell dilute solution can be obtained analytically. The constitutive equation of such system has a well-known solution, that results to be the same of a convected Jeffreys model, also known as the Oldroyd$B$ model [28]. Using such constitutive equation, the complex modulus of a Hookean-dumbbell dilute solution writes as follows:

$$
\begin{aligned}
& G^{\prime}=\frac{c k_{B} T \lambda_{H}^{2} \omega^{2}}{1+\left(\lambda_{H} \omega\right)^{2}} \\
& G^{\prime \prime}-\eta_{s} \omega=\frac{c k_{B} T \lambda_{H} \omega}{1+\left(\lambda_{H} \omega\right)^{2}}
\end{aligned}
$$

The BD algorithm employed to estimate the dynamic response of a suspension submitted to a smallamplitude oscillatory strain was previously described 
in $\S 2$. An explicit integration scheme is implemented in the BD simulation and the Kramers expression is employed to compute the shear stress tensor. Using a time step equal to $\lambda_{H} / 100$ and a population of $10^{3}$ dumbbells (convergent simulation parameters at no-flow conditions), the BD performance is checked in Fig. 8 by plotting the loss modulus at the characteristic frequency $\left(\omega=\lambda_{H}^{-1}\right)$ in function of the maximal imposed strain.

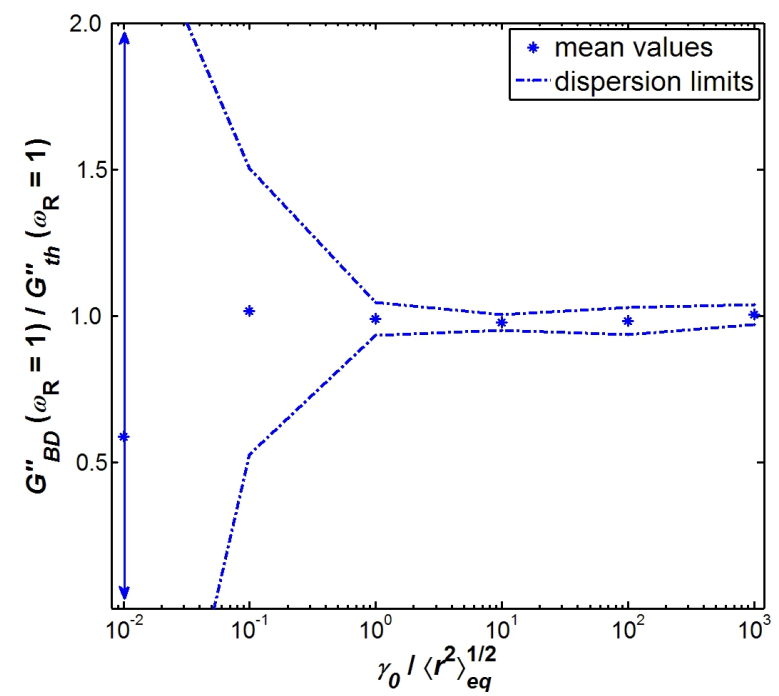

Fig. 8 BD prediction of the loss modulus at the characteristic frequency for a Hookean dumbbell model in function of the maximal shear strain applied. Maximal shear strain is normalized by the equilibrium end-to-end distance of a Hookean dumbbell model. $H, \zeta$ and $c k_{B} T$ are fixed to 1 in a consistent system of units. A population of $10^{3}$ dumbbells and a time step equal to $\lambda_{H} / 100$ have been employed

Bead-spring models are not able to describe the decrease of viscosity when shear rate is enhanced [28]. This fact is confirmed by Fig. 8 where the BD estimation of the loss modulus at the characteristic frequency appears independent of the maximal shear strain applied. However, a huge numerical dispersion is observed at low strains. This numerical noise is given by the stochastic nature of the Brownian forces when the magnitude of those ones is equal or higher than the magnitude of the flow-induced forces. In fact a narrower dispersion was obtained when implementing a more precise random number generation algorithm.

At low frequencies the convergence of the integration scheme is guaranteed by the criteria obtained for the no-flow conditions. That is truth because at low frequencies, system motion is controlled essentially by the thermal activity. At high frequencies, nevertheless, additional considerations are needed in order to satisfy the convergence of the integration scheme. In fact, at higher frequencies than the characteristic one, flow- induced forces become the controlling factor of the kinematics of the system and the relative importance of the stochastic forces is progressively reduced. For that reason at high frequencies, as the intensity of the flow field increases time step must to be gradually refined in order to guarantee convergence towards the central values. To show this fact, in Fig. 9 the BD convergence at two high frequencies $\left(\omega_{R}=10\right.$ and $\left.\omega_{R}=10^{2}\right)$ is deployed by plotting the relative error of the loss modulus estimation for a population of $10^{3}$ dumbbells with respect to the time step implemented.

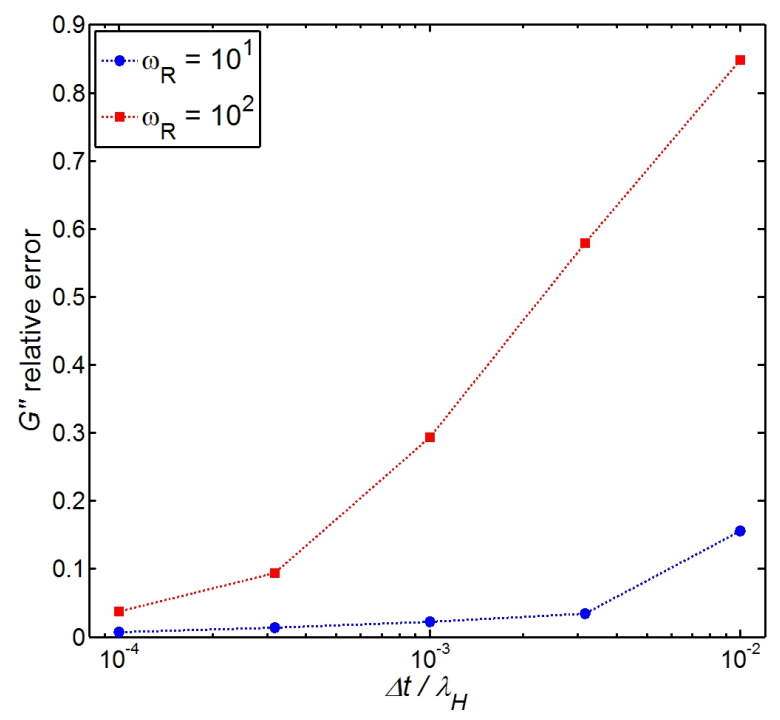

Fig. 9 Relative error of the BD prediction for the loss modulus at high frequencies in function of the time step. $H, \zeta$ and $c k_{B} T$ are fixed to 1 in a consistent system of units. A population of $10^{3}$ dumbbells has been employed. Relative error is defined as follows: $\left|\left(\log G_{B D}^{\prime \prime}\left(\omega_{R}\right)-\log G_{t h}^{\prime \prime}\left(\omega_{R}\right)\right) / \log \Theta\right|$, where $\Theta=G_{t h}^{\prime}\left(\omega_{R}=10^{2}\right) / G_{t h}^{\prime \prime}\left(\omega_{R}=10^{2}\right)$ is the maximal interval of complex modulus variation in the tested frequency interval

From Fig. 9, at a reciprocal frequency equal to $10^{1}$, a diminution of the relative error from $16 \%$ to $0.7 \%$ is appreciated when the time step is reduced from $\lambda_{H} / 100$ to $\lambda_{H} / 10^{4}$. An even more pronounced effect of the time step is observed for a reciprocal frequency of $10^{2}$, given the high sensitivity of the sine function around zero (phase angle tends to zero as frequency increases). In that case, a diminution of the relative error from $84 \%$ to $4 \%$ is noticed for the same refining of the time step. On the other hand, storage modulus is much less sensitive to the time step than the loss modulus in the high frequency regime because of the less variability of the cosine function with respect to the sine function around zero. For example, at $\omega_{R}=10^{2}$, a reduction of the relative error going from $1.6 \%$ to less than $0.1 \%$ is 
observed when the time step is diminished from $\lambda_{H} / 100$ to $\lambda_{H} / 10^{4}$.

In Fig. 10 the global BD performance to predict the complex modulus in a frequency sweep test within a representative frequency interval is presented.

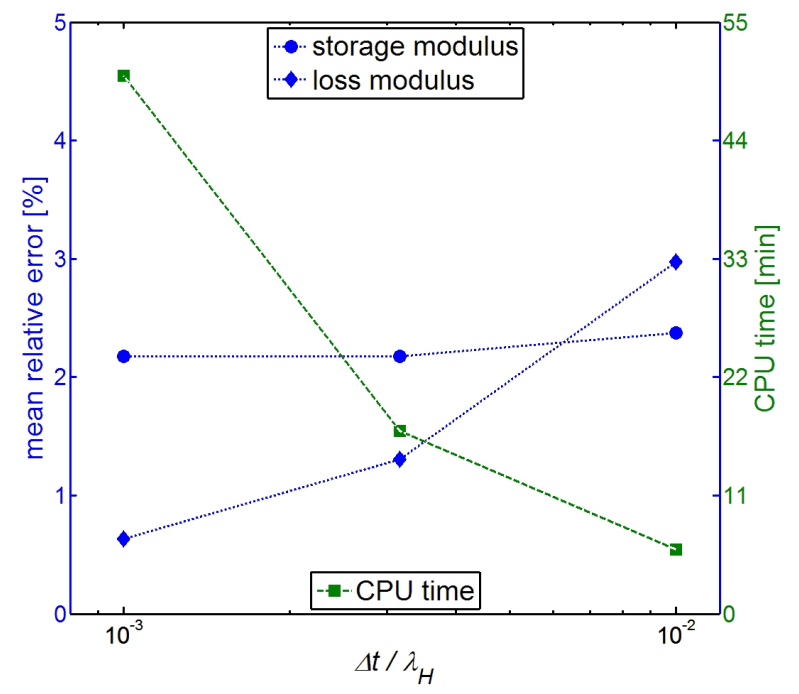

Fig. 10 Mean relative error of the BD prediction of the storage and loss modulus (for a Hookean dumbbell model) and total computational time in function of the time step implemented. Mean relative errors are calculated as the average of 34 points distributed homogenously in a reduced frequency range going from $10^{-2}$ to $10^{2}$. Relative error is defined as follows: $\left|\left(\log G_{B D}^{\prime}\left(\omega_{R}\right)-\log G_{t h}^{\prime}\left(\omega_{R}\right)\right) / \log \Theta\right|$, where $\Theta=G_{t h}^{\prime}\left(\omega_{R}=10^{2}\right) / G_{t h}^{\prime}\left(\omega_{R}=10^{-2}\right)$ is the maximal interval of complex modulus variation in the tested frequency interval. $H, \zeta$ and $c k_{B} T$ are fixed to 1 in a consistent system of units. A population of 5000 dumbbells has been employed

$\mathrm{BD}$ performance is depicted by plotting the mean relative error for the storage and loss modulus for 34 frequencies homogenously distributed within an interval of reduced frequencies going from $10^{-2}$ to $10^{2}$ in function of the time step. A population of 5000 dumbbells is used in the BD simulations. In all the sweep frequency tests a practically constant mean relative error for the storage modulus is obtained $(\sim 2 \%)$. This inaccuracy in the prediction of the storage modulus is coming mainly from the low frequencies regime $\left(10^{-2} \leq \omega_{R} \leq 10^{-1}\right)$ given the high variability of the cosine function approaching $\pi / 2$ (phase angle tends to this value as frequency diminishes). No improvement in convergence to the central values is appreciated when refining the time step because the origin of the dispersion is not associated with the integration scheme, but with the natural fluctuations of the stochastic forces that control the kinematics of the dumbbell at low velocities. Implemen- tation of a more sophisticated algorithm for generating random numbers is required to improve the convergence of the storage modulus at low frequencies. In fact, the list of pseudo-random numbers generators is continuously growing as reflect of the remaining challenges in this computational art $[3,70]$. On the contrary, a considerable improvement in the loss modulus prediction is checked as smaller time steps are employed. A reduction of the mean relative error from $3.0 \%$ to $0.6 \%$ is obtained when time step is shortened from $\lambda_{H} / 100$ to $\lambda_{H} / 10^{3}$. This improvement in convergence for the loss modulus is related directly to the better accuracy at high frequencies, as showed before in Fig. 9. As expected, a linear increment on the computational time

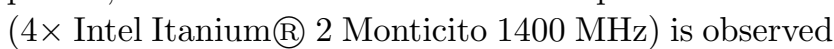
as the time step is shortened.

\subsubsection{The Multi-Bead-Spring Chain}

The analytical expression of the constitutive equation for the Rouse model (composed of $n-1$ Hookean springs) is well-known. It results to be the linear superposition of $n-1$ Hookean dumbbells with a spectrum of relaxation times $\lambda_{j}$ following the next normal modes [28]:

$\lambda_{j}=\frac{\zeta / 2 H}{4 \sin ^{2}(j \pi / 2 n)}$

When a dilute solution of Rouse chains is submitted to a small-amplitude oscillatory deformation test, the complex modulus contribution given by the chains writes as follows:

$G^{\prime}=c k_{B} T \sum_{j=1}^{n-1} \frac{\lambda_{j}^{2} \omega^{2}}{1+\left(\lambda_{j} \omega\right)^{2}}$
$G^{\prime \prime}-\eta_{s} \omega=c k_{B} T \sum_{j=1}^{n-1} \frac{\lambda_{j} \omega}{1+\left(\lambda_{j} \omega\right)^{2}}$

In principle, the numerical considerations extracted from the BD simulations with Hookean-dumbbells can be extrapolated to the BD simulations of multi-beadspring chains. In Figs. 11 and 12 the BD predictions for the complex modulus of Rouse chains with 1, 10 and 50 springs are compared. All BD simulations are carried out using a population of 5000 chains, a number proved to provide a good compromise between prediction accuracy and computational time (see Fig. 6). A frequency sweep test is carried out on 34 points homogeneously distributed in a reciprocal frequency interval going from $10^{-1}$ to $10^{2}$. A constant time step equal to $\lambda_{n-1} / 500$ was implemented, where $\lambda_{n-1}$ is the shortest time of the relaxation times spectra. This length of the time step was demonstrated to provide also a good 
compromise between accuracy and computational cost (see Fig. 10).

From Fig. 11 is observed that the prediction of the storage modulus is less accurate at low frequencies. It was already mentioned that the inaccuracy at low frequencies comes from the random number generation; hence improvement in the accuracy of the BD prediction is subjected to the implementation of more sophisticated algorithms of random number generation. Quality of the random number generation is only reflected at low frequencies because, at this regime of flow, the dynamics of the multi-bead-spring chain is governed by the randomizing thermal forces; whereas at high frequencies the statistical errors of the computational random number generation are masked by the effects of the external-imposed flow-field.

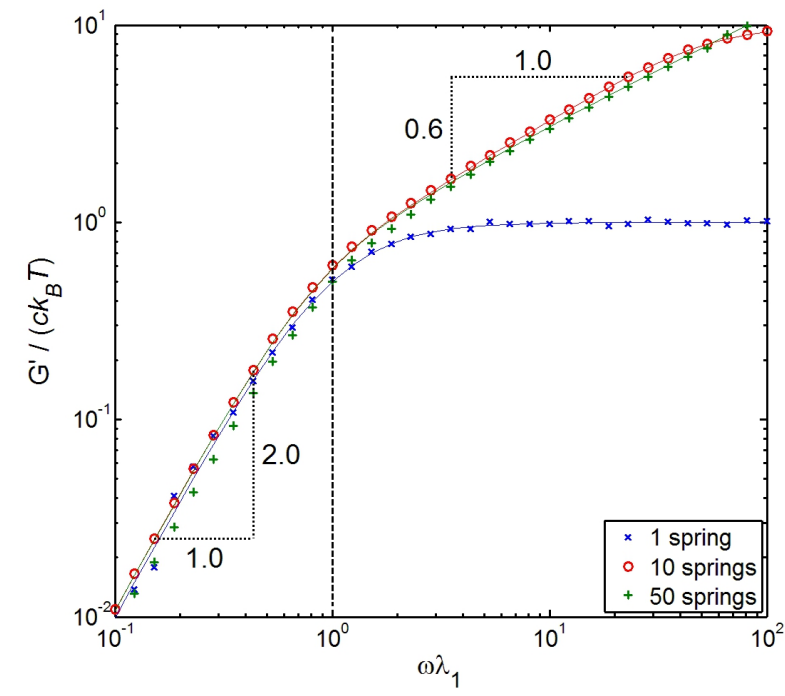

Fig. 11 BD prediction of the storage modulus in function of the reduced frequency for three Rouse chains: 1-spring, 10springs and 50-springs. Analytical curves are represented by solid lines. $H, \zeta$ and $c k_{B} T$ are fixed to 1 in a consistent system of units. A population of 5000 chains has been employed

Mean relative errors for the $\mathrm{BD}$ prediction of the storage modulus are $0.9 \%, 0.2 \%$ and $0.1 \%$ for the single Hookean dumbbell, the 10-springs and the 50-springs chain respectively. BD simulations are capable to predict the apparition of new relaxation processes when increasing the number of springs. Notice the elastic enhancement at high frequencies, going from the characteristic plateau of the Hookean dumbbell model to non-zero slopes of the storage modulus in function of the frequency; for instance 0.6 for the 10-springs and 50 -springs chain around a reduced frequency of 10 . It is worth to mention that Rouse theory predicts a storage modulus evolving with $\omega^{0.5}$ for very long chains
( $n>300)$. Tendency towards this limiting value has been registered by the BD simulations; a slight reduction of the storage modulus slope in the high frequency range is appreciated when going from 0.65 for the $10-$ springs chain to 0.61 for the 50-springs one.

In Fig. 12 the BD predictions of the loss modulus in function of the frequency are plotted for the same Rouse chains presented in the Fig. 11. For the Hookean dumbbell system, inaccuracy in the BD prediction is concentrated at high frequencies, on the contrary of the storage modulus. In this case, given the low values of loss modulus at high frequencies, error is coming from a bad resolution of the integration scheme when the phase angle approaches 0 . Shorter time steps are required in this frequency window $\left(10^{1}\right.$ to $\left.10^{2}\right)$, but it is important to say that the model response at those frequencies lacks of physical meaning too.

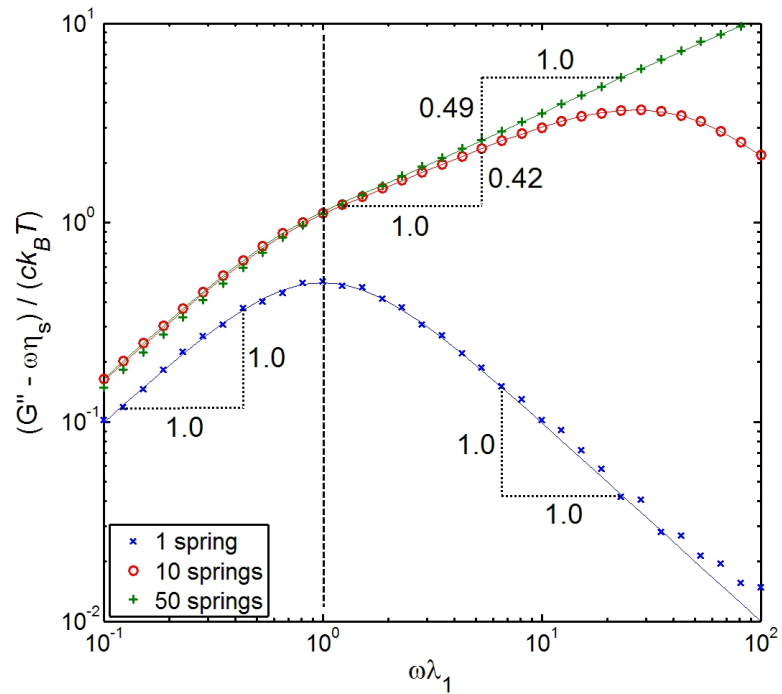

Fig. 12 BD prediction of the loss modulus in function of the reduced frequency for three Rouse chains: 1-spring, 10springs and 50-springs. Analytical curves are represented in solid lines. $H, \zeta$ and $c k_{B} T$ are fixed to 1 in a consistent system of units. A population of 5000 chains has been employed

BD simulations are able to correctly predict the evolution of the loss modulus in the high frequency range. Observe, for instance, the linear decrease of the loss modulus for the dumbbell system and the linear increases of the loss modulus for the 10-springs and 50springs chains at high frequencies. In the high frequency range, the predicted slopes of 0.42 and 0.49 for the 10springs and 50-springs chains respectively, are also consistent with the maximal limiting value of 0.5 given by the Rouse theory. BD simulations show that when increasing the number of springs, storage and loss moduli tend converging towards the same values in the high 
frequency range, as predicted by Rouse theory for large number of springs $(n>300)$.

Mean relative errors for the loss modulus prediction are $1.6 \%, 0.3 \%$ and $0.7 \%$ for the Hookean dumbbell, the 10-springs and the 50-springs chain respectively. $\mathrm{CPU}^{1}$ time $(4 \times$ Intel Itanium $\AA 2$ Monticito $1400 \mathrm{MHz})$ goes up from 15 minutes to 10 hours when passing from 1 -spring to 10-springs, an impressive increment of the computational cost. This fact is explained by the longer main relaxation times when dealing with bigger number of springs that are traduced in longer stabilization steps in the BD algorithm given in $\S 2$, specially in the high frequency range. Additionally, the short time steps required in an explicit integration scheme imply longer analytical steps in the implemented BD algorithm, specially in the low frequency range.

\subsection{The Zimm Model}

Unlike the Rouse model, in Zimm theory hydrodynamic interaction is taken into account. From a formal point of view, the kinematic description is exactly the same as that one of the Rouse model, except for the hydrodynamic drag force, where an additional term is considered to account for the local variation in the bulk flow field caused by the motion of the other beads in the multi-bead-spring chain. Pioneer works on hydrodynamic interaction inside flexible macromolecules are attributed to Kirkwood and Riseman [71,30]. Zimm adapted the Kirkwood's results in hydrodynamic interaction to the multi-bead-spring model in order to estimate the viscoelastic, birefringence and dielectric properties of dilute polymer solutions. Zimm found the analytical solution of the model by using a transformation to normal coordinates [58].

The particularities of the implementation of a BD simulation for a multi-bead-spring model with hydrodynamic interaction can be revealed by transforming the general expression for the bead velocity given in Eq. (28). Neglecting external forces and supposing an isotropic friction tensor, the generalized stochastic differential equation in Eq. (28) can be rewritten as follows:

$\frac{d \mathbf{r}_{i}}{d t}=-\sum_{j} \boldsymbol{\Omega}_{i j} \cdot \mathbf{F}_{j}^{(h)}+\left[\boldsymbol{\kappa}\left(\mathbf{r}_{i}\right) \cdot \mathbf{r}_{i}\right]+\frac{\mathbf{F}_{i}^{(\phi)}}{\zeta}+\frac{\mathbf{F}_{i}^{(b)}}{\zeta}$

where the local hydrodynamic interaction term $\mathbf{v}_{i}^{\prime}$ has been approximated by using the Oseen-Burgers hydrodynamic tensor. As the balance of forces over each bead

1 Central Processing Unit is zero (inertialess Langevin equation) the hydrodynamic force in Eq. (56) can be written in terms of the other forces:

$$
\begin{aligned}
\frac{d \mathbf{r}_{i}}{d t}= & \sum_{j} \boldsymbol{\Omega}_{i j} \cdot\left[\mathbf{F}_{j}^{(\phi)}+\mathbf{F}_{i}^{(b)}\right]+\left[\boldsymbol{\kappa}\left(\mathbf{r}_{i}\right) \cdot \mathbf{r}_{i}\right] \\
& +\frac{\mathbf{F}_{i}^{(\phi)}}{\zeta}+\frac{\mathbf{F}_{i}^{(b)}}{\zeta}
\end{aligned}
$$

Reordering and bringing together common terms:

$$
\begin{aligned}
\frac{d \mathbf{r}_{i}}{d t}= & {\left[\boldsymbol{\kappa}\left(\mathbf{r}_{i}\right) \cdot \mathbf{r}_{i}\right]+\frac{1}{\zeta} \sum_{j} \mathbf{H I}_{i j} \cdot \mathbf{F}_{j}^{(\phi)} } \\
& +\frac{1}{\zeta} \sum_{j} \mathbf{H I}_{i j} \cdot \mathbf{F}_{j}^{(b)}
\end{aligned}
$$

where $\mathbf{H I}_{i j}=\delta_{i j} \boldsymbol{\delta}+\zeta \boldsymbol{\Omega}_{i j}$ is called the Hydrodynamic Interaction (HI) matrix associated with a given pair of beads $i$ and $j$ inside the chain. Finally, through the fluctuation-dissipation theorem the stochastic differential Eq. (58) for the bead velocity in a multi-bead-spring chain with hydrodynamic interaction takes the form [1]:

$$
\begin{gathered}
\frac{d \mathbf{r}_{i}}{d t}=\left[\boldsymbol{\kappa}\left(\mathbf{r}_{i}\right) \cdot \mathbf{r}_{i}\right]+\frac{1}{\zeta} \sum_{j} \mathbf{H I}_{i j} \cdot \mathbf{F}_{j}^{(\phi)}+ \\
\sqrt{\frac{2 k_{B} T}{\zeta}} \sum_{j} \mathbf{C}_{i j} \cdot \frac{d \mathbf{W}_{j}}{d t}
\end{gathered}
$$

where $\mathbf{H I}_{i j}=\mathbf{C}_{i j} \cdot \mathbf{C}_{i j}^{T}$. Hydrodynamic interaction in multi-bead-spring chains is tackled rigorously with Eq. (59), but at present it cannot be solved in closed form [1]. To circumvent this difficulty, Zimm theory replaces the variable Oseen-Burgers tensors by their equilibrium averages. By using the equilibrium-averaged distance between two beads inside a Rouse chain $\left\langle r_{i j}\right\rangle_{e q}$ (see [28]) the equilibrium-averaged Oseen-Burgers hydrodynamic tensor given in $\S 3.1$ can also be expressed as follows [58]:

$$
\left\langle\boldsymbol{\Omega}_{i j}\right\rangle_{e q}=\frac{1-\delta_{i j}}{6 \pi \eta_{s}} \sqrt{\frac{2 H}{\pi k_{B} T|i-j|}} \boldsymbol{\delta} \text { for } i \neq j
$$

When introducing Eq. (60) into Eq. (59) it is found that $\mathbf{H I}_{i j}=\mathbf{H I}$ and $\mathbf{C}_{i j}=\mathbf{C}$ where $\mathbf{H I}=\mathbf{C} \cdot \mathbf{C}^{T}$. As noticed, in Zimm theory HI tensor becomes a $n \times n$ constant matrix whose components $(\mathbf{H I})^{i j}$ are given by the next formula:

$$
(\mathbf{H I})^{i j}=\delta_{i j}+\left(1-\delta_{i j}\right) h^{*} \sqrt{\frac{2}{|i-j|}}
$$

where $h^{*}$ is the hydrodynamic interaction parameter according to Thurston and Peterlin [72]:

$h^{*}=\frac{\zeta}{\eta_{s}} \sqrt{\frac{H}{36 \pi^{3} k_{B} T}}$ 
where $\zeta$ is the bead friction coefficient, $\eta_{s}$ is the solvent viscosity and $H$ is the Hookean-spring constant. The hydrodynamic interaction parameter $h^{*}$ can be expressed also as the ratio of the bead radius to the rootmean-square distance between two beads linked by a spring at equilibrium. In that context, it is expected that $h^{*}$ is lower than $0.5[1]$. From a practical point of view, experimental viscoelastic data for several polymer solutions are generally well represented by Zimm chains of several hundreds of beads with hydrodynamic interaction parameters between 0.1 and 0.2 [73].

From a numerical point of view (BD approach) integration of the stochastic differential equation for a Zimm chain (Eq. (59)) does not represent an additional cost with respect to the Rouse chains (Zimm hydrodynamic interaction matrix is constant). In addition, equations employed to describe the kinematics and to compute the shear-stress tensor are quite similar for both models. Hence, the numerical considerations about the number of trajectories required and the time step (in an explicit integration scheme) inferred for Rouse chains can, in principle, be extended to the BD simulations of Zimm chains.

It seems that the calculation of the matrix $\mathbf{C}_{i j}$ has a numerical relevance when general hydrodynamic interaction (i.e. based on the general expression of the Oseen-Burgers tensor) for chains with large number of beads $(n>300)$ is considered, owing to the fact that the hydrodynamic interaction matrix is recalculated at each time step. Classical Cholesky decomposition is expensive, scaling with the cube of the number of beads [1]. In response, Fixman proposed two alternative numerical methods to calculate the square-root of the hydrodynamic interaction matrix: one by Newton iteration and the other one by Chebyshev polynomial decomposition [74]. Implementation of the last polynomial approximation for the square-root of the hydrodynamic interaction matrix in a BD framework is detailed elsewhere [1]; this method roughly scales with $n^{9 / 4}$ per time step. In any case, previous numerical issue has a reduced impact on the BD simulation of Zimm chains due to the fact that the equilibrium-averaged hydrodynamic interaction matrix is constant and the required decomposition is carried out just one time for all the simulation.

Given the fact that hydrodynamic interaction is approximated by the equilibrium averaged Oseen-Burgers tensor, a close-form of the constitutive equation can be derived for the Zimm model in total analogy with that one of the Rouse model [28,3]. For that reason, expressions for the contribution of Zimm chains to the complex modulus of their dilute suspensions have the same form that the equations for Rouse chains (Eqs. (54) and (55)):

$$
\begin{aligned}
& G^{\prime}=c k_{B} T \sum_{j=1}^{n-1} \frac{\tilde{\lambda}_{j}^{2} \omega^{2}}{1+\left(\tilde{\lambda}_{j} \omega\right)^{2}} \\
& G^{\prime \prime}-\eta_{s} \omega=c k_{B} T \sum_{j=1}^{n-1} \frac{\tilde{\lambda}_{j} \omega}{1+\left(\tilde{\lambda}_{j} \omega\right)^{2}}
\end{aligned}
$$

The only difference lies on the relaxation time spectra. In the Zimm model the time constants are dictated by:

$\tilde{\lambda}_{j}=\frac{\zeta}{2 H \tilde{a}_{j}}$

where $\tilde{a}_{j}$ are the eigen-values of the modified Rouse matrix $\tilde{\mathbf{A}}$ whose components $(\tilde{\mathbf{A}})^{i j}$ are given by:

$$
(\tilde{\mathbf{A}})^{i j}=\sum_{v} \sum_{u} \bar{B}_{i v}(\mathbf{H I})^{v u} \bar{B}_{j u}
$$

and $\bar{B}_{v u}$ is a scalar operator following the next formula [28]:

$\bar{B}_{v u}=\delta_{v+1 u}-\delta_{v u}$

This analytical solution allows us to evaluate easily the performance of the BD simulation predicting the linear viscoelastic behaviour of multi bead-spring systems with equilibrium-averaged hydrodynamic interaction. Figures 13 and 14 compare the BD predictions of the complex modulus for a 50-springs Zimm chain with two different hydrodynamic interaction parameters. A frequency sweep test is carried out over 25 points homogeneously distributed in a reduced frequency interval going from $10^{-1}$ to $10^{2}$.

From Fig. 13 is observed that the BD prediction of the storage modulus is more accurate for the high hydrodynamic interaction case than the low one. In fact, the mean relative error for the low value of $h^{*}$ is about $2.7 \%$ in comparison with the mean relative error of $0.8 \%$ for the case of high $h^{*}$. The main source of inaccuracy is coming from the low frequency range. This is explained, as in the BD simulations with Rouse chains, by the statistical deviations originated in the numerical computation of the stochastic forces coupled with the relative dominance of the thermal forces as driving mechanism of the chain kinematics in the low frequency range. As a consequence, improvement of the accuracy at low frequencies depends on the implementation of more sophisticated random numbers generators.

On the other hand, BD simulations correctly predict the evolution of the storage modulus at high frequencies. Figure 13 shows the increase of the slope of $G^{\prime}$ going from 0.6 for the low hydrodynamic interaction case 


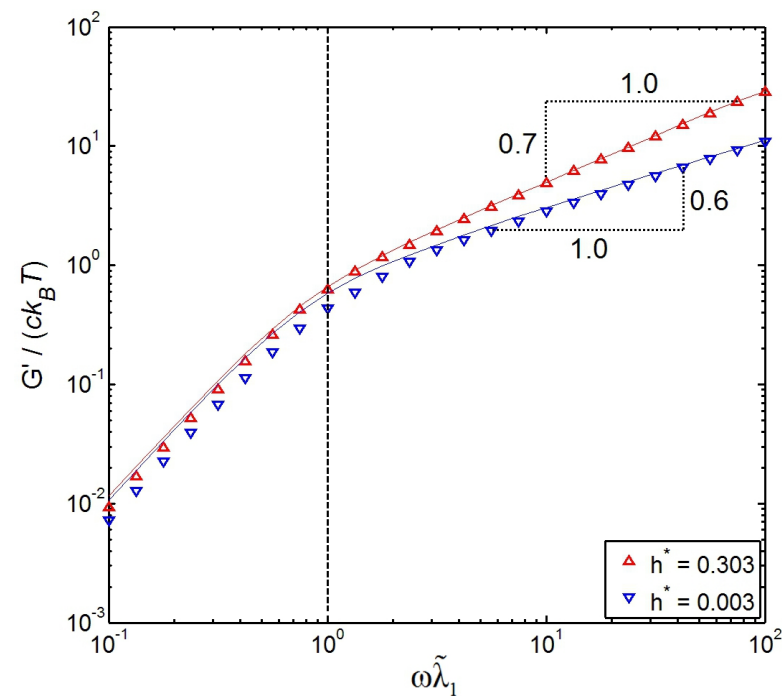

Fig. 13 BD prediction of the storage modulus in function of the reduced frequency for two 50-springs Zimm chains with high hydrodynamic interaction $\left(h^{*}=0.303\right)$ and very low hydrodynamic interaction $\left(h^{*}=0.003\right)$. Analytical curves are represented in solid lines. $H, \zeta$ and $c k_{B} T$ are fixed to 1 in a consistent system of units. A population of $10^{4}$ dumbbells has been employed

$\left(h^{*}=0.003\right)$ to 0.7 for the high hydrodynamic interaction case $\left(h^{*}=0.303\right)$, within the reduced frequency interval between $10^{1}$ and $10^{2}$.

In Fig. 14 the BD prediction of the loss modulus versus the frequency is plotted for the same Zimm chains presented in Fig. 13. Again better accuracy is obtained for the chain with higher hydrodynamic interaction. In fact, mean relative error goes down from $1.6 \%$ to near $0.3 \%$ when $h^{*}$ is increased from 0.003 to 0.303 . As observed for the storage modulus prediction, main source of error is coming from the low frequency regime.

BD performance at high frequencies is quite better. Simulations are able to correctly predict the change in the loss modulus slope when varying the relative importance of the hydrodynamic interaction. At low hydrodynamic interaction a Rouse-type slope is observed, whereas at high hydrodynamic interaction $\left(h^{*}=0.303\right)$, the slope of the loss modulus reaches a maximal value of 0.64 around a reduced frequency of 10 . This prediction is consistent with the Zimm theory for very long chains (i.e. $n>300$ ) and high hydrodynamic interaction (for instance, $h^{*}=0.3$ ), where storage and loss moduli evolve linearly with $\omega^{2 / 3}[75]$. On the other hand, in our BD simulation of 50-springs chains with high hydrodynamic interaction $\left(h^{*}=0.303\right)$ we have found $\left(G^{\prime \prime}-\eta_{s} \omega\right) / G^{\prime}=1.67$ around a reduced frequency of 10 , a result also in coherence with Zimm theory where a constant ratio $\left(G^{\prime \prime}-\eta_{s} \omega\right) / G^{\prime}=\sqrt{3}$ is predicted in the high frequency range.

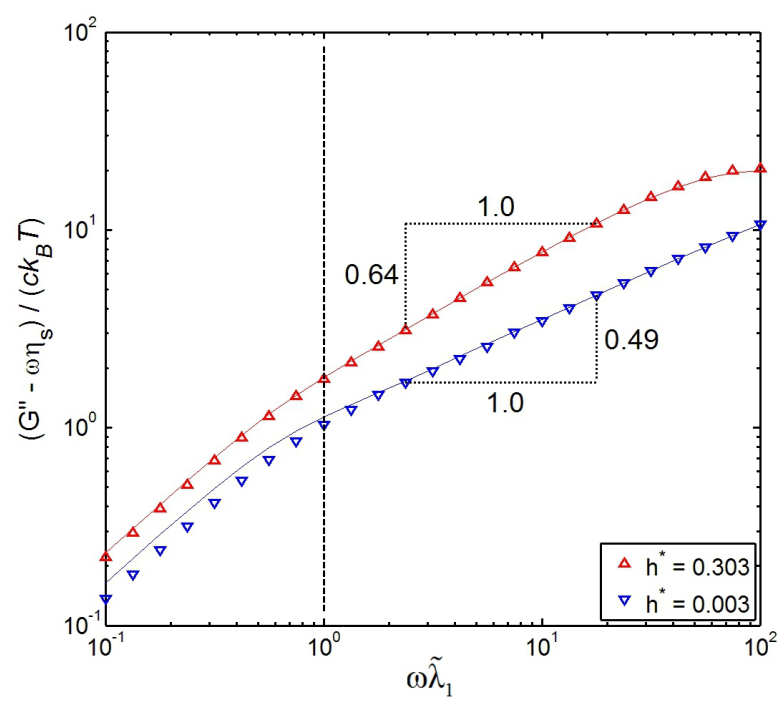

Fig. 14 BD prediction of the loss modulus in function of the reduced frequency for two 50-springs Zimm chains with high hydrodynamic interaction $\left(h^{*}=0.303\right)$ and very low hydrodynamic interaction $\left(h^{*}=0.003\right)$. Analytical curves are represented in solid lines. $H, \zeta$ and $c k_{B} T$ are fixed to 1 in a consistent system of units. A population of $10^{4}$ dumbbells has been employed

\section{BD of Generalized Bead-Rod Models}

The fundamental difference between the bead-rod model and the bead-spring model lies in that the former has a finite contour length. This simple, but important fact is traduced directly in different rheological responses. On the other hand, a physical model with a finite contour length imposes subtle, but important numerical issues that are reflected in the BD implementation.

As mentioned in §1.2.1, historically bead-rod models were first proposed to emulate the structure of linear polymers in a coarse-grained fashion. Bead-rod model with fixed adjacent angles (as proposed by Flory [29]) constitute the first stage of coarse-graining in kinetic theory. In that model, rods are supposed to represent the bond length between two consecutive atoms in the backbone of the polymer chain. Simpler representations with fewer degrees of freedom have been extensively proposed. For instance, some polymer chains are quite bendable along certain number of monomer units, in those cases from 3 to 5 monomer units can be replaced by one non-bendable rod. Polymer flexibility is then mimicked by introducing some kind of bending potential between consecutive rods. Continuous version of this model is known as the worm-like chain, in which the thermal equilibrium configuration is a perfect straight filament. An even coarser model replaces from 10 to 20 monomer units by one rigid-rod; any notion of flexion hindering at this rod-scale is lost and the poly- 
mer chain is represented then by a freely-jointed multirod chain [28]. An ultimate scale in the coarse-graining process replaces a hundred of backbone atoms (or a ten of rods from the freely-jointed multi-rod model) by an entropic spring, so mechanical behaviour of the macromolecule is represented by a multi-bead-spring chain model (showed in the previous section §3). Despite that the historical motivation for the bead-rod model is linked to the polymer science, this representation has been extensible applied to emulate the mechanical behaviour of other structures as short DNA, rod-like virus, collagen fibrils, synthetic polymers and CNTs.

Bead-rod models have escaped from a deeply development in the kinetic theory framework with respect to the bead-spring counterparts because the inextensibility condition imposes the use of generalized coordinates [76]. This mathematical complexity explains why only some results in zero-shear, steady and linear unsteady flows have been obtained for this model $[77,78]$.

In the case of bead-rod models, BD simulations appear as an interesting alternative approach to by-pass the complex mathematical-treatment of the diffusion equation. Anyway, the use of constraints also implies to be careful during a BD implementation, particularly in terms of the integration scheme and the stochastic forces generation.

Bead-rod models have been particularly studied for approaching the mechanical behaviour of bead-spring chains with very stiff Fraenkel-type springs (non-zero natural length). Intuitively a very stiff Fraenkel spring could be replaced by a rigid rod for the sake of mathematical simplicity. However, it is found that this limit is a very singular one. In fact, bead-rod chains and beadspring chains differ even in the limit of infinitely stiff springs. For instance, the configurational distribution of a freely-jointed multi-bead-rod chain at thermal equilibrium is found to be different from a random-walk distribution, which is typical for a freely-jointed multibead-spring chain [28]. This difference has been confirmed by molecular and Brownian dynamics simulations $[79,80,81,5]$ and statistical mechanics calculations $[82,83]$. On the other hand, it has been showed that introducing a corrective potential force (function of the chain configuration) into the multi-bead-rod chain formulation is possible to mimic the behaviour of multibead-spring chains $[82,84]$. This result strongly promoted the use of BD simulations for studying the dynamical behaviour of polymers by using the multi-beadrod model and the corrective potential approach $[66,74$, $85,86,87,88,89]$. In this work, the intrinsic behaviour of multi-bead-rod models is tackled; the bead-spring chain statistics is not intended to be mimicked.
In what follows, the basis of a BD implementation for a generalized multi-bead-rod chain with an isotropic friction tensor and in absence of hydrodynamic interaction is presented. We focus in two classical cases: the freely-jointed chain or Kramers model and the non freely jointed chain that corresponds to a discrete version of the worm-like chain model.

\subsection{Kinematic and Dynamic Formulation}

Constraints can be treated mathematically in a generalized coordinate system (based on the configuration of the chain) or in a Cartesian coordinate system, case in which constraint forces are required to complement the kinematic description. Going and coming back between those two frameworks requires a lot of care in order to conserve a proper equivalence at the levels of the FP equation and the differential stochastic equation [3].

From a formal point of view a general, expression of the FP equation for a multi-bead-rod model with no internal potentials, including hydrodynamic interaction and anisotropic friction tensor, was given by Curtiss using generalized coordinates [28]. This diffusion equation is coherent with the equilibrium statistical mechanics for a real bead-rod chain with equal bead masses. A detailed description of the development of an equivalent stochastic differential equation in the strong sense was given by Öttinger [3]. He also showed a particular transformation of that differential stochastic equation from generalized to Cartesian coordinates. In doing so, not only an explicit definition of the rod inextensibility is required, but also a metric potential force, depending on the chain configuration, has to be considered in order to respect the equivalence (at least in a weak sense) with the FP equation developed by Curtiss. Interestingly, it is found that the negative of such metric force is equal to the corrective potential force added in the BD numerical algorithms of multi-bead-rod chains intended to mimic the behaviour of multi-bead-spring chains.

For the sake of simplicity, BD implementation in a Cartesian coordinate system is employed in this paper. Metric potential forces are neglected without incurring in error because, in one hand, the drag acting on the simulated multi-bead-rod chain is supposed characterized by an isotropic friction tensor and, on the other hand, no hydrodynamic interactions are considered.

Let us consider the multi-bead-rod chain model showed in Fig. 15. Multi-bead-rod chain is constituted of $n$ beads joined by $n-1$ non-bendable rigid rods of length a.

Position of the bead-rod chain can be instantaneously defined in a Cartesian reference system by the ensem- 


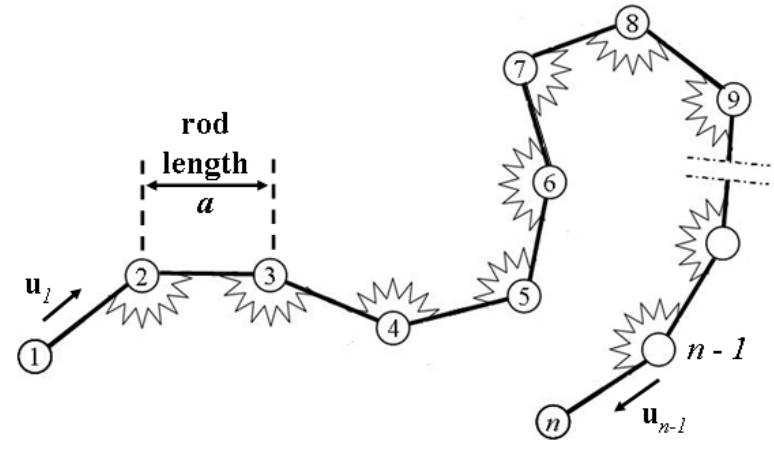

Fig. 15 Non-freely jointed multi-bead rod model composed of $n$ beads and $n-1$ rods of length $a$. Bending potential between rods in the multi-bead-rod chain model is mimicked with a hypothetical flexion spring

ble of bead positions $\mathbf{r}_{i}$ and the centre of mass of the chain $\mathbf{r}_{c}$ (see Eq. (14)). Orientation of each rod is given by the vector $\mathbf{u}_{j}$, which is a unit vector collinear to the rod connecting beads $j$ and $j+1$. $\mathbf{u}_{j}$ is defined as follows:

$\mathbf{u}_{j}=\left(\mathbf{r}_{j+1}-\mathbf{r}_{j}\right) / a$

Rods are supposed infinitely rigid. Physical length of the system is equivalent to the total length of the multi-bead-rod chain $(n-1) a$. Existence of an internal bending potential, coming from a hypothetical flexion spring between each pair of rods, is considered with the purpose of mimic the bending flexibility of the filamentlike system. Introduction of a non-zero bending potential complicates the FP formulation and make of the BD simulation a privileged numerical approach. On the other hand, multi-bead-rod chains are supposed suspended into a Newtonian solvent (viscosity $\eta_{s}$ ) at temperature $T$. Concentration of the bead-rod chains is defined in terms of $c$ chains per volume unit. High dilution hypothesis is made, so no inter-chain interaction is considered. Moreover, flow field acting on the suspension is assumed to be homogeneous. Beads are considered the centres of hydrodynamic resistance; so all forces are concentred on beads. In what follows, forces acting on the multi-bead-rod chains are described in more detail.

- Hydrodynamic drag force acting on bead $i, \mathbf{F}_{i}^{(h)}$ Physical origin and mathematical description of this force are the same given for the multi-bead-spring model (see §3.1). As mentioned before, hydrodynamic interaction is neglected and an isotropic friction coefficient is supposed in order to avoid the calculation of metric potential forces.

- Constraint forces acting on bead $i, \mathbf{F}_{i}^{(\lambda)}$

This force takes into account the sum of rod tensions acting on each bead $i$. Rod tension $\lambda_{j}$ corresponds to the module of the instantaneous force emerging on bead $j$ for maintaining the distance between beads $j$ and $j+1$ at a constant value $a$. This force is transmitted along the rigid-rod and is manifested on bead $j+1$ with an opposite sign. Mathematically, total constraint force on bead $i$ can be expressed as follows:

$\mathbf{F}_{i}^{(\lambda)}=-\sum_{j=1}^{n-1} \mathbf{n}_{i j} \lambda_{j}$

where $\mathbf{n}_{i j}$ is a linear operator defined as follows:

$\mathbf{n}_{i j}=\mathbf{u}_{j}\left(\delta_{i j+1}-\delta_{i j}\right)$

- Internal bending potential force acting on bead $i$, $\mathbf{F}_{i}^{(\phi)}$

Based on the viscoelastic theory of concentrated solutions of semi-flexible polymers [90], Pasquali and Morse defined a discrete bending potential for a multi-bead-rod model based on the worm-like chain continuous model [87]:

$\phi=-\frac{K_{b}}{a} \sum_{i=2}^{n-1} \mathbf{u}_{i} \cdot \mathbf{u}_{i-1}$

where $\phi$ is the internal-bending potential and $K_{b}$ is a bending rigidity constant. The bending potential defined in Eq. (71) can be understood as coming from the mechanical action of a torsion spring between two consecutive rods. In this case, local bending energy is proportional to the cosine of the internal angle between two consecutive rods. In other words, minimal internal bending energy state is achieved when all rods are completely aligned. Usually, this linear relationship is restricted to small flexion angles. Obviously, definitions of non-linear bending potentials are required for large deformation scenarios. Bending force acting on bead $k$ can be obtained as the derivate of the bending potential in Eq. (71) with respect to the position of bead $k$ :

$\mathbf{F}_{k}^{(\phi)}=-\frac{\partial \phi}{\partial \mathbf{r}_{k}}=\frac{K_{b}}{a} \sum_{i=2}^{n-1} \frac{\partial\left(\mathbf{u}_{i} \cdot \mathbf{u}_{i-1}\right)}{\partial \mathbf{r}_{k}}$

To simplify the derivate on the right side of Eq. (72), next identity is employed [87]:

$\frac{\partial}{\partial \mathbf{r}_{k}} \mathbf{u}_{i}=\frac{1}{a}\left(\delta_{k i+1}-\delta_{k i}\right)\left(\boldsymbol{\delta}-\mathbf{u}_{i} \otimes \mathbf{u}_{i}\right)$

- Brownian force acting on bead $i, \mathbf{F}_{i}^{(b)}$

As in all coarse-grained models, Brownian forces approach the change of momentum of a bead $i$ coming from the thermal activity of the solvent molecules surrounding bead $i$. Accounting for faster processes 
than the coarse-grained model motion, Brownian forces are computed as an stochastic process. In a model without constraints (free particles, beadspring chains), Brownian forces follow a normal distribution with first and second moments defined previously by Eqs. (25) and (26), respectively. However, as the generalized friction of the bead-rod system is modified by the presence of constraints, random forces must reflect the inextensibility of rods [86].

An algorithm proposed to tackle properly last issue requires that Brownian forces are geometrically projected random forces. Geometrical projection of random forces on bead-rod chains is absolutely necessary when the bead-spring statistics want to be mimicked (using of corrective potential forces and a mid-step integration algorithm) or an anisotropic friction tensor is considered [89].

On the other hand, when dealing with free-draining bead-rod chains in which each bead is characterized by an isotropic friction coefficient $\zeta$, geometrical projection of Brownian forces is redundant [89] and same kinematics is obtained by using unprojected Brownian forces. To show last fact (and present in parallel the structure of the geometrical projection algorithm), let us take the example of one single rigid rod embedded into a solvent bath at temperature $T$, no external flow is imposed. Supposing an isotropic friction coefficient, the force balances on the two mass-less beads write:

$$
\begin{aligned}
& -\zeta \dot{\mathbf{r}}_{1}-\mathbf{n}_{11} \lambda_{1}+\mathbf{F}_{1}^{(b)}=m_{1} \ddot{\mathbf{r}}_{1}=\mathbf{0} \\
& -\zeta \dot{\mathbf{r}}_{2}-\mathbf{n}_{21} \lambda_{1}+\mathbf{F}_{2}^{(b)}=m_{2} \ddot{\mathbf{r}}_{2}=\mathbf{0}
\end{aligned}
$$

In order to satisfy the inextensibility of the rigidrod, next condition has to be imposed over the bead velocities:

$0=\sum_{i=1}^{2} \dot{\mathbf{r}}_{i} \cdot \mathbf{n}_{i 1}=\left(\dot{\mathbf{r}}_{2}-\dot{\mathbf{r}}_{1}\right) \cdot \mathbf{u}_{1}$

Placing the bead velocities from Eqs. (74) and (75) into Eq. (76) we obtain:

$$
\frac{1}{\zeta}\left(-\lambda_{1} \mathbf{u}_{1}+\mathbf{F}_{2}^{(b)}-\lambda_{1} \mathbf{u}_{1}-\mathbf{F}_{1}^{(b)}\right) \cdot \mathbf{u}_{1}=0
$$

Equation (77) can be rewritten also as follows:

$$
2 \lambda_{1}=\sum_{i=1}^{2} \mathbf{F}_{i}^{(b)} \cdot \mathbf{n}_{i 1}=\left(\mathbf{F}_{2}^{(b)}-\mathbf{F}_{1}^{(b)}\right) \cdot \mathbf{u}_{1}
$$

Previous equation resumes the conditions imposed over the Brownian forces in order to satisfy the inextensibility condition. Two numerical algorithms for the random forces generation can be considered at this point. The first takes into account the contribution of random forces to the rod tensions and implies to calculate $\lambda_{1}$ at each time step from Eq. (78). In this case, random forces are generated like in the $\mathrm{BD}$ simulations of bead-spring models and the predictor-corrector integration scheme $(\S 4.2 .2)$ is required. In the second, contributions of random forces to the rod tension are neglected $\left(\lambda_{1}=0\right)$ and unconstrained random forces have to be projected in order to satisfy $0=\sum_{i=1}^{2} \mathbf{F}_{i}^{(b)} \cdot \mathbf{n}_{i 1}$. This last expression determines the starting point of the geometrical projection algorithm. In order to obtain projected random forces $\mathbf{F}_{i}^{(b)^{\prime}}$, it is necessary to substrate the "hard" component of the unconstrained Brownian forces $\hat{\eta}_{1}$ along the rod direction as follows:

$\mathbf{F}_{i}^{(b)^{\prime}}=\mathbf{F}_{i}^{(b)}-\mathbf{n}_{i 1} \hat{\eta}_{1}$

The "hard" component of the unconstrained Brownian forces can be obtained from the solution of the next equation [89]:

$\hat{G} \hat{\eta}_{1}=\sum_{i=1}^{2} \mathbf{F}_{i}^{(b)} \cdot \mathbf{n}_{i 1}$

where $\hat{G}=\sum_{i} \mathbf{n}_{i 1} \cdot \mathbf{n}_{i 1}=2$. From Eqs. (78) and (80) is easily inferred that $\hat{\eta}_{1}=\lambda_{1}$. Hence, projected Brownian forces are $\mathbf{F}_{1}^{(b)^{\prime}}=\mathbf{F}_{1}^{(b)}+\lambda_{1} \mathbf{u}_{1}$ and $\mathbf{F}_{2}^{(b)^{\prime}}=$ $\mathbf{F}_{2}^{(b)}-\lambda_{1} \mathbf{u}_{1}$. A graphic example of this projection in a bi-dimensional framework is showed in Fig. 16. Extension of previous algorithm for a multi-beadrod case is straightforward and has been detailed elsewhere $[85,86]$.

Nevertheless, as mentioned before, geometrical projection of Brownian forces for a constrained system with an isotropic friction tensor is redundant because, as observed for the one-rod example in Fig. 16 , projection of random forces has no consequences on the rod kinematics as hydrodynamic forces and rod velocity are not altered. In other words, considering a constant friction coefficient, rod kinematics depends exclusively on the orthogonal component of the Brownian forces with respect to the rod vector and this component is not modified by the geometrical projection algorithm.

- External forces acting on bead $i, \mathbf{F}_{i}^{(e)}$

As mentioned before, gravitational and electromagnetic forces can be considered in function of the particular scenario of modelling. In this work, external forces are neglected.

Taking into account that inertia is neglected, forces balance on each bead $i$ writes:

$\mathbf{F}_{i}^{(h)}+\mathbf{F}_{i}^{(\lambda)}+\mathbf{F}_{i}^{(\phi)}+\mathbf{F}_{i}^{(b)^{\prime}}+\mathbf{F}_{i}^{(e)}=\mathbf{0}$ 


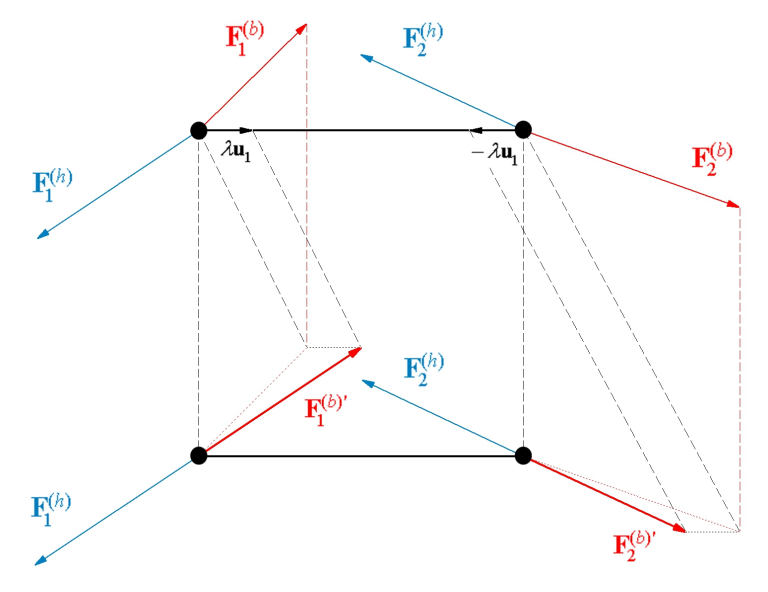

Fig. 16 Example of the geometrical projection of Brownian forces in a quiescent bi-dimensional single rigid-rod (no external-flow). An isotropic friction coefficient is supposed. In the upper rod no geometrical projection has been carried out, hence tension force is considered. In the lower rod, geometrical projection of Brownian forces absorbs the rod tension; observe that hydrodynamic forces are not modified

Given the definition of the hydrodynamic drag force, an explicit stochastic expression for the bead velocity can be obtained:

$\frac{d \mathbf{r}_{i}}{d t}=\left[\kappa\left(\mathbf{r}_{i}\right) \cdot \mathbf{r}_{i}\right]-\frac{1}{\zeta}\left\{\left(\begin{array}{c}n-1 \\ \left.\sum_{j=1}^{n} \mathbf{n}_{i j} \lambda_{j}\right)+\mathbf{F}_{i}^{(\phi)} \\ +\mathbf{F}_{i}^{(b)^{\prime}}+\mathbf{F}_{i}^{(e)}\end{array}\right\}\right.$

Geometrical projected random forces guarantee the inextensibility condition when no external fields are imposed. In other scenario, constraints on the rod lengths have to be considered. For instance, in a Cartesian coordinates system we have:

$0=\sum_{i=1}^{n} \dot{\mathbf{r}}_{i} \cdot \mathbf{n}_{i j} \quad$ for $\quad j=1 \ldots n-1$

Putting together Eqs. (82) and (83) a linear system of equations describing the kinematics of a non-freelyjointed multi-bead-rod model is constituted, where the unknown variables are $n$ bead velocities $\dot{\mathbf{r}}_{i}$ and $n-1$ rod tensions $\lambda_{j}$.

\subsection{Integration Schemes}

Several numerical integration schemes have been proposed to integrate the differential stochastic equation equivalent to the FP equation given by Curtiss for the general multi-bead-rod chain in both generalized coordinate systems and Cartesian reference systems. In the case of generalized coordinates, the task is feasible if the number of degrees of freedom is small, otherwise the numerical complexity makes this option impractical [3]. Some simulations at equilibrium using a numerical integration in generalized coordinates were made by Pear and Weiner $[81,5]$. The possibilities for integrating numerically in a Cartesian space are more varied and, in principle, the numerical methods employed for the bead-spring models are applicable, but additional controls are required for satisfying the constraints rigorously, specially in simulations for large time intervals [3].

Another integration numerical method for systems including constraints was given by Allison and McCammon under the name of SHAKE-HI algorithm [91]. In the general form of the algorithm, bead positions are calculated in two steps: an unconstrained step followed by a constrained one. Two particular versions of the SHAKE-HI algorithm are the most cited numerical schemes employed to integrate the differential stochastic equation for a multi-bead-rod model in a Cartesian space: the mid-point stepping scheme proposed by Fixman [82, $85]$ and the predictor-corrector scheme developed by Liu $[76,63]$.

\subsubsection{Mid-Point Algorithm}

In 1978 Ermak and McCammon showed that the gradient of the diffusivity must to be incorporated into a BD algorithm when the bead diffusivity depends on the configuration of the Brownian system [92]. It has been shown that the diffusivity of a multi-bead-rod chain is variable because of the presence of rigid constraints and, also, as consequence of considering hydrodynamic interaction [85]. A clever way to counteract the wrong drift produced by the variable diffusivity in a BD algorithm was proposed by Fixman [82]. He suggested employing a second-order time step to integrate the stochastic differential equation. In fact, any higher order time step is able to handle correctly the variable diffusivity. In this numerical algorithm the use of potential metric forces $\mathbf{F}_{i}^{(m)}$ is required and has to be added to the left term of Eq. (81). In brief, potential metric forces are function of the constrained configuration of the chain. Extended descriptions and detailed definitions for this "extra" metric force can be found elsewhere [84,3,89]. From a global point of view, this algorithm is able to deal with hydrodynamic interaction and anisotropic friction tensors. For the particular multi-bead-rod chain model presented in this work, the differential stochastic equation required for the mid-step scheme writes as follows:

$\dot{\mathbf{r}}_{i}=\frac{1}{\zeta}\left(\mathbf{F}_{i}^{(\lambda)}+\mathbf{F}_{i}^{(u c)}+\mathbf{F}_{i}^{(b)^{\prime}}\right)$

where $\mathbf{F}_{i}^{(u c)}=\zeta\left[\boldsymbol{\kappa}\left(\mathbf{r}_{i}\right) \cdot \mathbf{r}_{i}\right]+\mathbf{F}_{i}^{(\phi)}+\mathbf{F}_{i}^{(e)}+\mathbf{F}_{i}^{(m)}$. Keeping in mind that hydrodynamic interaction is neglected 
and an isotropic friction tensor is supposed, then a single time step $[t, t+\Delta t]$ for the mid-step algorithm is constituted by the following sub-steps:

1. Generation of geometrical projected random forces at the beginning of the time step $\mathbf{F}_{i}^{(b)^{\prime}}(t)$ following the complete algorithm describe by Hinch and coworkers $[84,85,86]$.

2. Calculate the mid-step position as follows:

$\mathbf{r}_{i}\left(t+\frac{\Delta t}{2}\right)=\mathbf{r}_{i}(t)+\dot{\mathbf{r}}_{i}(t) \frac{\Delta t}{2}$

where the initial-step velocity $\dot{\mathbf{r}}_{i}(t)$ is calculated neglecting the constraints on the rod lengths:

$\dot{\mathbf{r}}_{i}(t)=\frac{1}{\zeta}\left(\mathbf{F}_{i}^{(u c)}(t)+\mathbf{F}_{i}^{(b)^{\prime}}(t)\right)$

3. Calculate the end-step position by using the next equation:

$\mathbf{r}_{i}(t+\Delta t)=\mathbf{r}_{i}(t)+\dot{\mathbf{r}}_{i}(*) \Delta t$

where the mid-step velocity is obtained from the solution of the linear system:

$$
\begin{aligned}
& \dot{\mathbf{r}}_{i}(*)=\frac{1}{\zeta}\left[\begin{array}{l}
\mathbf{F}_{i}^{(\lambda)}\left(t+\frac{\Delta t}{2}\right)+ \\
\mathbf{F}_{i}^{(u c)}\left(t+\frac{\Delta t}{2}\right)+\mathbf{F}_{i}^{(b)^{\prime}}(t)
\end{array}\right] \\
& 0=\sum_{i=1}^{n} \dot{\mathbf{r}}_{i}(*) \cdot \mathbf{n}_{i j}\left(t+\frac{\Delta t}{2}\right)
\end{aligned}
$$

where $\dot{\mathbf{r}}_{i}(*)$ is the constrained bead velocity at the mid-step, but calculated with the random stochastic forces generated at the beginning of the time step. This procedure is coherent with the Stratonovich interpretation of the stochastic random forces [3, 93]. This algorithm does not guarantee an invariable rod length at the end of the time step, for that reason a truncation error is employed. Once the rod length at the end of a given time step exceeds certain tolerance, the inter-bead distances are reset to the constant value, conserving the rod orientations.

\subsubsection{Predictor-Corrector Scheme}

The predictor-corrector scheme is a singular and limiting case of the SHAKE-HI algorithm. One can say that is singular because it requires absolutely neglecting the hydrodynamic interaction and dealing with an isotropic friction tensor. On the other hand, this algorithm is limiting because the two-step procedure is merged in an iterative single-step. The predictor-corrector scheme was employed by Liu for simulating the dynamics of a Kramers chain submitted to steady potential flows [76]. Doyle demonstrated that both the predictor-corrector scheme and the Fixman's mid-step algorithm generate the same trajectories for a dilute Kramers beadrod chain with an isotropic friction tensor and in absence of hydrodynamic interaction [93]. It is emphasized that neglecting the hydrodynamic interactions, supposing an isotropic friction tensor and employing this limiting case of the SHAKE-HI algorithm are necessary conditions for eliminating the metric potential forces from the differential stochastic equation in Cartesian coordinates without incurring in deviations from the FP equation for the multi-bead-rod model [3].

For a time step $[t, t+\Delta t]$, displacement of bead $i$ is obtained in two stages. The first one, called predictor step, is given by an unconstrained movement:

$\mathbf{r}_{i}^{*}(t+\Delta t)=\mathbf{r}_{i}(t)+\dot{\mathbf{r}}_{i}(t) \Delta t$

where $\dot{\mathbf{r}}_{i}(t)=\frac{1}{\zeta}\left(\mathbf{F}_{i}^{(u c)}(t)+\mathbf{F}_{i}^{(b)^{\prime}}(t)\right)$ and $\mathbf{F}_{i}^{(u c)}=\zeta\left[\boldsymbol{\kappa}\left(\mathbf{r}_{i}\right) \cdot \mathbf{r}_{i}\right]+$ $\mathbf{F}_{i}^{(\phi)}+\mathbf{F}_{i}^{(e)}$. Subsequently, an iterative corrector step is required for taking into account the inextensibility of rods:

$\mathbf{r}_{i}(t+\Delta t)=\mathbf{r}_{i}^{*}(t+\Delta t)+\frac{1}{\zeta} \mathbf{F}_{i}^{(\lambda)} \Delta t$

where $\mathbf{F}_{i}^{(\lambda)}$ is given by Eq. (69). The Lagrange multipliers $\lambda_{i}$ are calculated so that inter-bead distances are satisfied within a certain tolerance:

$\left(\mathbf{r}_{i+1}(t+\Delta t)-\mathbf{r}_{i}(t+\Delta t)\right)^{2}-a^{2}=\varepsilon^{2}$

Combining Eqs. (91) and (92), a system of quadratic equations for $\lambda_{i}$ is generated. This system has the general form:

$\frac{2 \Delta t}{\zeta} \mathbf{b}_{i} \cdot\left(\lambda_{i-1}^{[n]} \mathbf{u}_{i-1}-2 \lambda_{i}^{[n]} \mathbf{u}_{i}+\lambda_{i+1}^{[n]} \mathbf{u}_{i+1}\right)=$ $a^{2}+\varepsilon^{2}-\mathbf{b}_{i} \cdot \mathbf{b}_{i}-$

$\left(\frac{\Delta t}{\zeta}\right)^{2}\left(\lambda_{i-1}^{[n-1]} \mathbf{u}_{i-1}-2 \lambda_{i}^{[n-1]} \mathbf{u}_{i}+\lambda_{i+1}^{[n-1]} \mathbf{u}_{i+1}\right)^{2}$

where $\mathbf{b}_{i}=\mathbf{r}_{i+1}^{*}-\mathbf{r}_{i}^{*}$ and $\lambda_{i}^{[n]}$ is the $n$th approximation of the Lagrange multiplier associated to $\operatorname{rod} i$. Previous set of non-linear equations can be solved iteratively by a Picard's method by supposing the non-linear term (last term on the right-hand side) to be small in comparison with the linear terms (left-hand side). The iterative procedure is initiated with $\lambda_{i}^{[0]}=0$ and is executed until the constraints are satisfied with regard to a certain truncation error $\varepsilon^{2}$. Once the iterative procedure has finished, the bead positions at the end of the time step are corrected using Eq. (91).

Additional to the Picard's method invoked to solve Eq. (93), an iterative Newton's method can converge faster than the Picard's one, but involving the calculation of a Jacobian matrix every time step [63]. 


\subsection{Stress Tensor Calculation}

From a formal point of view, deduction of a stress formula for bead-rod chains is not as straightforward as in the case of bead-spring models, where the physical configuration is an intimate reflect of the instantaneous mechanical state of the system. In the case of bead-rod chains, the pictorial arguments used for the bead-spring models are not enough and, in consequence, a deeper physical explanation is required.

There is a general consensus accepting that the modified Kramers expression (Eq. (46)) accounts for the contribution to the total shear stress coming from a diluted model system whose total change in momentum can be discretized on certain points, as is the case for a general bead-rod-spring model. A detailed derivation of this stress tensor formula using a general phase-space kinetic theory can be examined in chapters 17 and 18 of [28].

Care must be taken also when calculating numerically the stress tensor for systems containing constraints. Given the numerical singularities originated by the presence of constraints, one is particularly interested in the correct implementation of a stress tensor formula into the $\mathrm{BD}$ algorithm. It is found that a correct estimation of the stress tensor must to be closely linked to the integration scheme used in the simulation.

In order to account rigorously for the Brownian effects on the stress tensor, the algorithm estimating the stress tensor must to consider the discontinuity of the Brownian forces during a given time step [86,93], specially when no-flow conditions are being simulated. To do this, the modified Kramers formula has to be interpreted as an average in time. For a mid-step algorithm, for example, the average has to be carried out using the beginning and the end of the time step [94]:

$\boldsymbol{\tau}_{p}(t)=\frac{c}{2} \sum_{v=1}^{n}\left\langle\begin{array}{l}\mathbf{R}_{v}(t) \otimes \mathbf{F}_{v}^{(h)}(t)+ \\ \mathbf{R}_{v}(t+\Delta t) \otimes \mathbf{F}_{v}^{(h)}(t+\Delta t)\end{array}\right\rangle$

Doyle demonstrated that two iterations of the predictorcorrector scheme producing an increment of $\Delta t$ are equivalent to one time-step of the mid-step algorithm [93]. Based on the previous fact, it can be easily showed that Eq. (94) is also a correct interpretation of the virial tensor for the predictor-corrector algorithm.

An alternative way to correctly account for the Brownian contribution in a first order integration scheme (as the predictor-corrector algorithm), considering a "longlasting" action of the random forces, can be envisaged using the next algorithm:

1. Update the bead-rod position by executing one explicit time step from $t$ to $t+\Delta t$.
2. Using the same Brownian forces used at time $t$, recalculation of the hydrodynamic drag forces $\tilde{\mathbf{F}}_{v}^{(h)}(t+\Delta t)$ with the updated configuration at $t+$ $\Delta t$.

3. Determination of the shear-stress tensor at time $t$ by computing:

$$
\boldsymbol{\tau}_{p}(t)=c \sum_{v}\left\langle\mathbf{R}_{v}(t+\Delta t) \otimes \tilde{\mathbf{F}}_{v}^{(h)}(t+\Delta t)\right\rangle
$$

In previous algorithm Brownian forces are interpreted in the Stratonovich sense, which is equivalent to stating that the stochastic forcing occurs throughout the entire time step [93]. This interpretation of the Brownian forces is particularly important in the systems containing constraints because has direct consequences in both integration schemes (as observed clearly in the mid-step algorithm) and stress tensor calculations. A simple example showing the impact of the interpretation of the Brownian nature in the shear-stress tensor calculation is given next. Consider a diluted 2D fibre aligned with the $x$ axis at time $t$ as show in Fig. 17 . Friction acting on beads is supposed isotropic. No external flow is considered, only Brownian and drag forces take place.

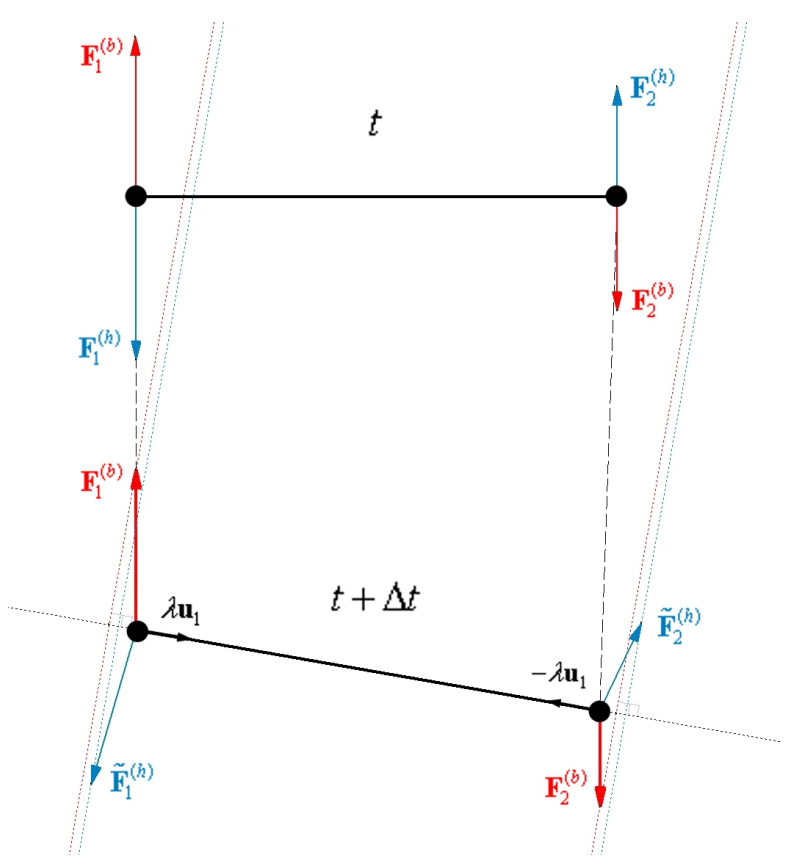

Fig. 17 Example of the "long-lasting" action of Brownian forces over a quiescent 2D single rod throughout a time step. An isotropic friction coefficient is supposed. Upper rod corresponds to the configuration at time $t$, lower rod corresponds to the configuration at time $t+\Delta t$. Observe that hydrodynamic forces at the end of the time step present non-null projections on the fibre axis 
It can be easily demonstrated, from the example of the geometrical projection of Brownian forces given in $\S 4.1$, that effective random forces in such configuration only occurs perpendicularly to the fibre axis, explaining why Brownian forces depicted in Fig. 17 at time $t$ are perpendicular to fibre axis. If Brownian forces acting at time $t$ are interpreted as instantaneous and stress tensor calculation is based exclusively on the mechanical balance obtained at time $t$ then the contribution of the fibre to the shear-stress tensor would be null $\left(\tau_{p}=\mathbf{0}\right)$, as hydrodynamic forces $\mathbf{F}_{v}^{(h)}(t)$ are always perpendicular to the connector vectors $\mathbf{R}_{v}(t)$.

Suppose now that Brownian forces at time $t$ occurs continuously until the end of the time step $t+\Delta t$ as showed in Fig. 17, where Brownian forces conserve magnitude and direction for the updated fibre configuration at time $t+\Delta t$. In that case, the contribution of the fibre to the shear-stress must include the configuration and mechanical balance of the fibre at the end of the time step. By employing the "long-lasting" Brownian forces algorithm, the shear-stress tensor contribution of a representative ensemble of fibres initially aligned with the $x$ axis converges to the next form:

$\tau_{p}=\alpha\left(\begin{array}{cc}1 & 0 \\ 0 & -1\end{array}\right)$

where $\alpha$ is a constant function of the temperature. Previous shear-stress tensor is in coherence with the results in theoretical mechanics for a quiescent rigid fibre immersed in a solvent, in which fibre experiences tension along its axis and compression in the normal direction.

Symmetry is another important issue when using the Kramers-Kirkwood expression for estimating the contribution of a given micro-mechanical model to the shear-stress tensor. Diluted bead-rod chains submitted to a homogeneous velocity field, in absence of external forces $\mathbf{F}_{v}^{(e)}$, are intended to produce a symmetric contribution to the total shear-stress. In a BD approach, symmetry is reached from the average of progressively bigger populations of bead-rod chains and/or the use of improved random number generators. In our BD simulation, limited by the size of the BD system, the estimated shear-stress tensor at each time $\tau_{p}(t)$, is made symmetrical in order to calculate the rheological properties following the next formula:

$\left[\boldsymbol{\tau}_{p}(t)\right]_{s y m}=\frac{1}{2}\left(\left[\boldsymbol{\tau}_{p}(t)\right]+\left[\boldsymbol{\tau}_{p}(t)\right]^{T}\right)$

As expected, convergence of the previous expression to the central values of shear-stress strongly depends on the size of the BD population. For example, considering the $2 \mathrm{D}$ rigid-fibre aligned with the $x$ axis presented previously, in Fig. 18 the progressively convergence of the out-of-diagonal component of the shear-stress tensor can be appreciated as the size of the BD population is increased.

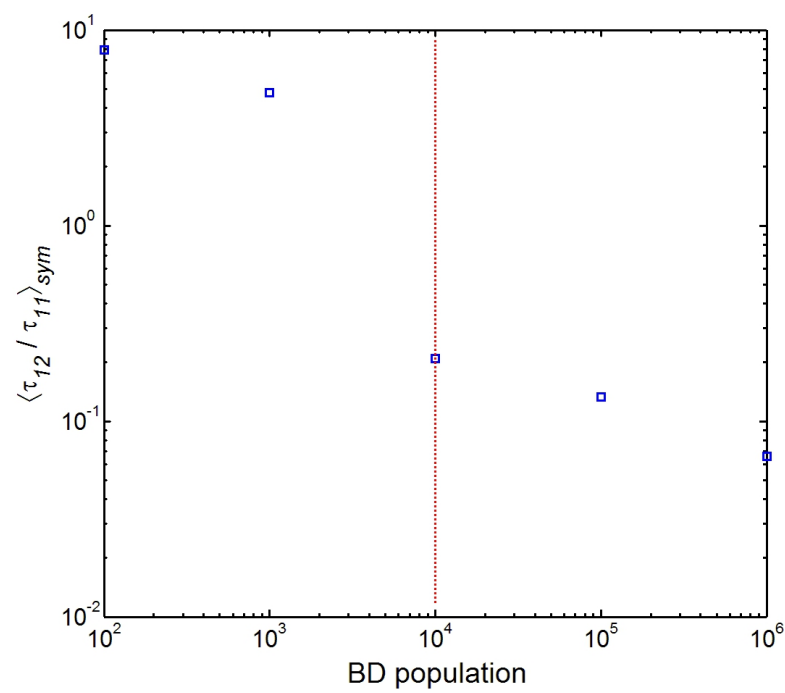

Fig. 18 Ratio between the out-of-diagonal and diagonal components of the first time step shear-stress tensor in function of the $\mathrm{BD}$ population of $2 \mathrm{D}$ rigid-fibres initially aligned with the $x$ axis. Shear-stress tensor has been made symmetrical by Eq. (97). Vertical dotted line marks the minimum required population for obtaining acceptable convergence to the central values in absence of external flow. Consider that in the current case the ratio $\left\langle\tau_{12} / \tau_{11}\right\rangle_{\text {sym }}$ is theoretically equal to zero

Notice that, in absence of flow, at least a population of $10^{4}$ systems is required to converge to the central values. Otherwise, aberrant values for the out-of-diagonal component of the shear-stress tensor can be obtained.

\subsection{The Trimer Chain}

In order to study the generalities of the BD simulations for multi-bead-rod models it was decided to use the freely-jointed (three-beads)-(two-rods) model, also known as the trumbbell or trimer system. This model is composed of three beads with isotropic friction coefficient $\zeta$ connected by two freely jointed rigid massless rods of length $a$. Hydrodynamic interaction is neglected.

Trimer chain is a better testing model than the rigid dumbbell because a more rigorously evaluation of the integration algorithms can be carried out given the presence of two adjacent constraints. On the other hand, an exact numerical expression for the dynamic response of a dilute solution of trimer systems was obtained separately by Fixman and Hassager [95,77]. This 
numerical solution is in coherence with the FP equation for a multi-bead-rod chain in polymer kinetic theory $[67,28]$, actually deviated from that one for multi-beadspring chains [80]. In the Hassager's solution, dynamic modulus of the trimer system is described using four relaxation times $\lambda_{i}^{H}$ of which the longest one writes:

$\lambda_{1}^{H}=\frac{\zeta a^{2}}{5.4376 k_{B} T}$

With the aim to compare properly with the BD predictions, time scales for the theoretical data are rescaled using $\lambda_{1}^{H}$.

In a trimer chain hydrodynamic interaction is neglected and friction is supposed isotropic. For that reason, a first-order integration scheme in coherence with the predictor corrector algorithm can be implemented in the BD simulation of trimer chains avoiding the use of metric potential forces. In this paper, shear-stress tensor contribution is estimated with the modified KramersKirkwood expression using the "long-lasting" Brownian forces algorithm. From now on, bi-dimensional BD simulations are implemented because we have found that this modelling correctly approaches the analytic solution of the FP equation for a trimer system. Time scale in the BD simulations is made dimensionless using the longest relaxation time (or rotational diffusion time) of an equal-length ( $n$-bead)-(rigid-rod) system given by [28]:

$\lambda_{n}=\frac{\zeta L^{2} n(n+1)}{72(n-1) k_{B} T}$

where $L=a(n-1)$. It means that for a trimer system, where $n=3$, time is scaled using $\lambda_{3}=\zeta a^{2} / 3 k_{B} T$. Time step, instead, needs to be scaled with regard to the shortest relaxation time; bead diffusion time is normally employed, $\lambda_{\text {bead }}=\zeta a^{2} / k_{B} T$.

As showed in the analysis of the Hookean-dumbbell model ( $\S 3.4 .1)$, the BD considerations when estimating the equilibrium properties (in absence of flow) are analogues to those ones obtained under linear dynamic solicitation at the characteristic frequency, i.e. at $\omega_{R}=1$. For that reason, no-flow properties are skipped and linear dynamic response of the system is studied directly.

$\mathrm{BD}$ simulations of the small-amplitude oscillatory deformation tests are carried out according to the algorithm explained in $\S 2$. First of all, the limit of the linear viscoelastic regime needs to be determined. In order to extract this information from the $\mathrm{BD}$ simulations, the norm of the complex modulus at the characteristic frequency is plotted in function of the maximal applied strain. In order to marginalize the numerical issues of the BD simulation, a population of $10^{4}$ chains and a time step equivalent to $\lambda_{\text {bead }} / 10^{5}$ are employed.
Figure 19 shows that the limit of the linear viscoelastic regime for a dilute solution of trimer systems corresponds to a maximal deformation $\gamma_{0} \approx 1.33 a$. At lower values, dynamic moduli obtained by BD simulations are independent of the maximal deformation imposed. On the other hand, Fig. 19 evidences that multibead-rod models exhibit a shear-thinning behaviour, contrary to the multi-bead-spring counterparts. Using a constant $\mathrm{BD}$ population $\left(10^{4}\right.$ trimers $)$ and a constant time step $\left(\lambda_{\text {bead }} / 10^{5}\right)$, computational time $(2 \times$ QuadCore AMD Opteron $\left.{ }^{\mathrm{TM}} 2376(2300 \mathrm{MHz})\right)$ employed to predict the complex modulus at $\omega_{R}=1$ was found 8.7 times longer than the computational time used at $\omega_{R}=10^{2}$. This fact is explained by the higher number of time steps required at low frequencies for sweeping one-and-a-half period of oscillation and determining the maximal shear-stress and phase angle between strain and stress signals.

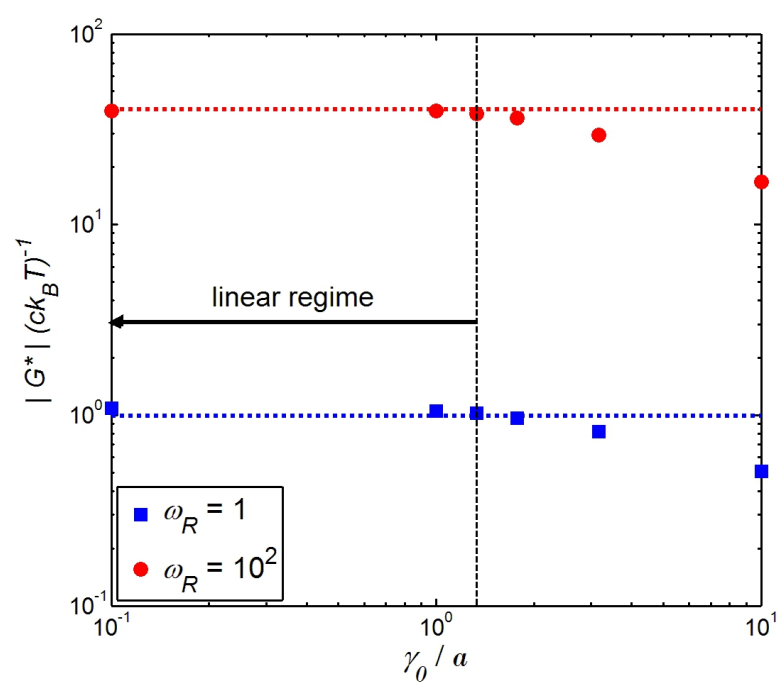

Fig. 19 Identification of the limit of the linear viscoelastic regime for a dilute suspension of trimers by BD simulation. Absolute value of the complex modulus in function of the maximal deformation applied for two reduced frequencies: $\omega_{R}=1$ and $\omega_{R}=10^{2}$. Horizontal dotted lines correspond to the theoretical values calculated by Hassager. Dashed vertical line marks the limit of the linear viscoelastic regime

In the current $\mathrm{BD}$ approach, a first-order integration scheme was implemented and, therefore, time step strongly influences the convergence of the simulation towards the central values. In Fig. 20 the effect of the time step on the convergence of the $\mathrm{BD}$ simulation is presented (from now on, imposed maximal deformation is chosen inside the linear viscoelastic regime). $10^{4}$ trimers were employed as $\mathrm{BD}$ system in order to reduce the distortions coming from the random number generation. Convergence of the BD simulation is tested 
in three different flow scenarios: $\omega_{R}=1$, which is representative of the thermal-to-flow induced transition, $\omega_{R}=10^{2}$, which is characteristic of the mild flowinduced regime, and, $\omega_{R}=10^{3}$, which is typical of a strongly flow-induced regime.

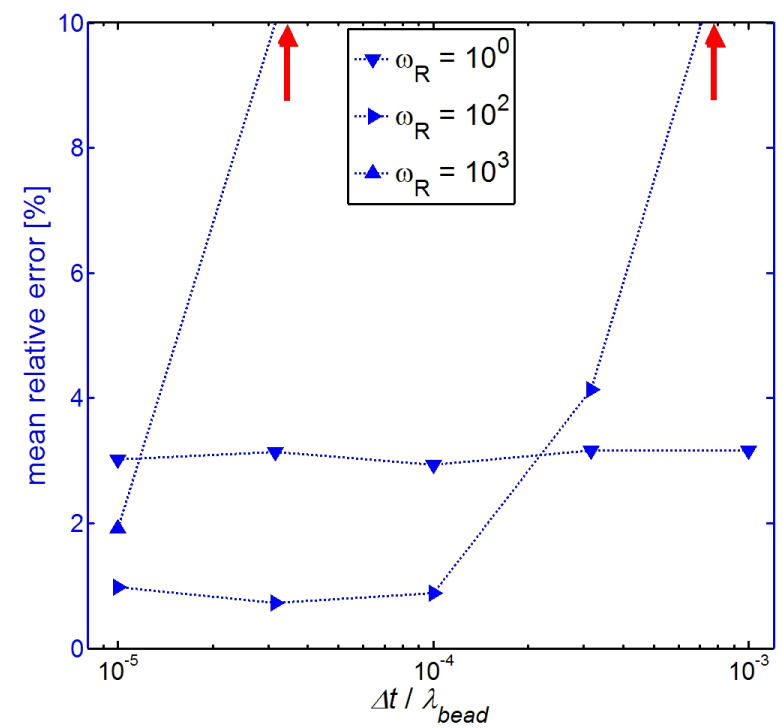

Fig. 20 Mean relative error of the BD prediction of the complex modulus for a trimer system in function of the time step. Mean relative error is the average of the relative errors for storage and loss modules. Relative error is defined as follows: $\left|\left(\log G_{B D}^{\prime}\left(\omega_{R}\right)-\log G_{t h}^{\prime}\left(\omega_{R}\right)\right) / \log \Theta\right|$, where $\Theta=G_{t h}^{\prime \prime}\left(\omega_{R}=10^{3}\right) / G_{t h}^{\prime}\left(\omega_{R}=1\right)$ is the maximal interval of complex modulus variation in the tested frequency interval. A population of $10^{4}$ trimers has been employed

In Fig. 20 is clearly observed the strong influence of the length of the time step in the convergence of the explicit integration scheme at high frequencies. At low frequencies $\left(\omega_{R}=1\right)$, characteristic of the thermalinduced regime, $\mathrm{BD}$ performance (relative error $\sim 3 \%$ ) is nearly independent of the length of the time step. In this regime, convergence of the integration scheme is guaranteed with a time step equal to $\lambda_{\text {bead }} \cdot 10^{-3}$. Error obtained at this flow regime is mainly coming from the random number generation algorithm; implementation of a more sophisticated generator is necessary to reduce this noise. When increasing the frequency, BD performance is progressively more affected by the integration scheme. As showed for the multi-bead-spring models, at higher frequencies than the characteristic one, the flowinduced forces control the kinematics of the system; for that reason, higher velocities require smaller time steps in order to guarantee a correct numerical integration. This fact is clearly captured in Fig. 20: in the mild flow-induced regime, i.e. $\omega_{R}=10^{2}$, mean relative error of the $\mathrm{BD}$ prediction goes from $1 \%$ to $12 \%$ when the length of the time step is enlarged from $\lambda_{\text {bead }} \cdot 10^{-5}$ to $\lambda_{\text {bead }} \cdot 10^{-3}$. In the case of strong flow-induced regime, i.e. $\omega_{R}=10^{3}$, mean relative error of the BD prediction explodes from $2 \%$ to more than $20 \%$ when the length of the time step is augmented just in one-half order of magnitude from $\lambda_{\text {bead }} \cdot 10^{-5}$.

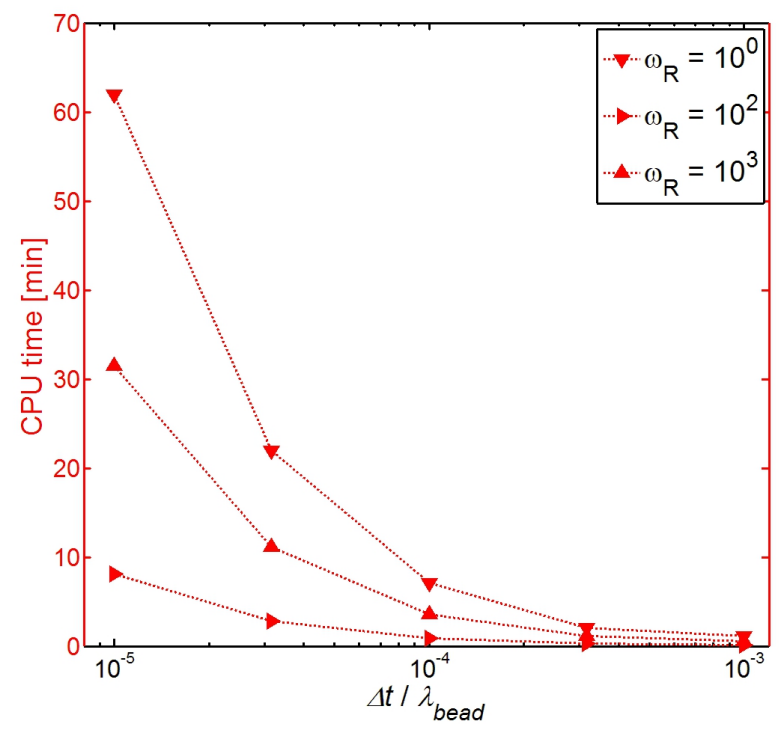

Fig. 21 CPU time required to predict complex modulus for a trimer system at three different frequencies $\left(\omega_{R}=1, \omega_{R}=\right.$ $10^{2}$ and $\omega_{R}=10^{3}$ ) in function of the time step. A population of $10^{4}$ trimers has been employed

On the other hand, in Fig. 21 is observed that simulation time is reduced as length of the time step is enlarged. Moreover, for a given value of time step, computational time used for calculating complex modulus strongly depends on the tested frequency; for instance, when implementing $\Delta t=\lambda_{\text {bead }} \cdot 10^{-5}$, simulations last in minutes $62.0,8.1$ and 31.5 for reduced frequencies of $1,10^{2}$ and $10^{3}$ respectively. Simulation at the characteristic frequency has the biggest computational cost because it requires the longest analytical step (1.5 periods of oscillatory deformation at the lowest frequency). Moreover, simulation at the highest frequency is also time-consuming because an additional refining of the nominal time step is required for well describe the oscillatory deformation signal.

In what follows, an extended sweep test over 21 frequencies homogeneously distributed within an interval of reduced frequencies going from $10^{-2}$ to $10^{3}$ was used to evaluate the global performance of the implemented BD simulation predicting the linear dynamic response of a trimer system in dilute solution. A time step equal to $\lambda_{\text {bead }} \cdot 10^{-5}$ has been employed to guarantee the best convergence of the integration scheme inside the simu- 
lated frequency range. In Fig. 22 the Hassager's numerical solution is compared with the BD predictions for the frequency sweep test. Hassager's complex modulus is presented in a reduced frequency scale $\omega_{R}=\omega \lambda_{1}^{H}$, and $\mathrm{BD}$ results are plotted in a reduced frequency scale $\omega_{R}=\omega \lambda_{3}$, where $\lambda_{3}$ is the rotational diffusion time for a (3-beads)-(rigid-rod) system of equal length.

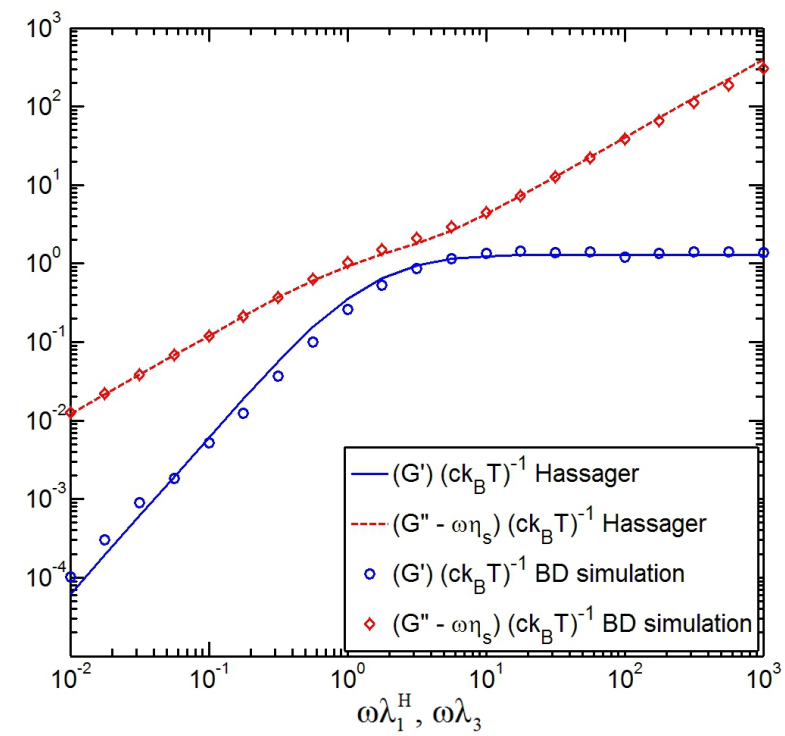

Fig. 22 Dynamic modulus of a freely jointed (three-beads)(two-rods) system in a proper reduced frequency scale: comparison between the Hassager's numerical solution and the current BD simulation. $\lambda_{1}^{H}$ is the main relaxation time in the Hassager's solution and $\lambda_{3}$ is the rotational diffusion time of a system composed of (3-beads)-(rigid-rod) with equal length. $a$ and $\zeta$ are fixed to $1, c k_{B} T$ to 0.1 and $K_{b}$ to 0 in a consistent system of units

BD algorithm yielded a complex modulus in good agreement with the Hassager's numerical solution of the trimer system as observed in Fig. 22. On the other hand, this large-sweep simulation demonstrated that a bi-dimensional modelling is able to correctly approach the 3D analytical solution given by Hassager and, therefore, avoids implementing a three-dimensional modelling, which would be more expensive in computational time. Two different regions are clearly differentiated in the dynamic response of a trimer system: a low frequency regime exhibiting a typical Maxwell terminal behaviour, where the thermal activity (or Brownian forces) drives the kinematic of the system; and a high frequency regime, where the domination of flow forces over the diffusivity terms produces a plateau for the storage modulus and a limiting constant viscosity $(G(\omega) / \omega)-\eta_{s}$ larger than zero. The existence of a non-zero limiting viscosity is physically more coherent than the classical responses of multi-bead-spring chains, where the contribution to the dynamic viscosity disappears at high frequencies.

As already mentioned in the section devoted to the bead-spring models, the number of entities (or trajectories) plays a fundamental role in the performance of the BD simulations. Performance of the global simulation in function of the BD population is presented in Fig. 23. The performance of the BD simulation is measured in terms of the mean of the relative errors for 17 storage modulus values homogeneously distributed in an interval of reduced frequencies going from $10^{-2}$ to $10^{2}$. Loss modulus signature was found practically insensitive to the BD population; mean relative error for the loss modulus signal was equal to $0.3 \%$.

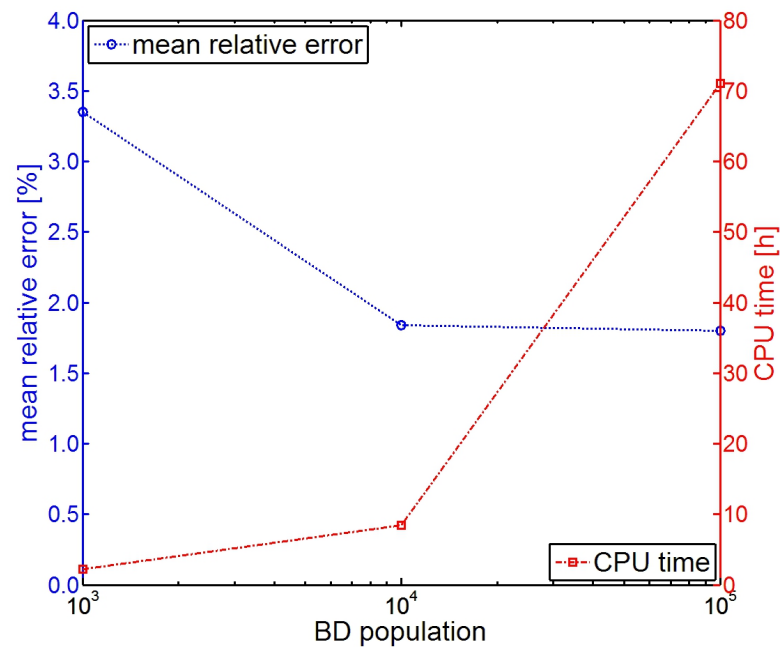

Fig. 23 Mean relative error of the storage modulus prediction (for a trimer system) and total computational time in function of the BD population. Mean relative errors are calculated as the average of 17 points distributed homogenously in a reduced frequency range going from $10^{-2}$ to $10^{2}$. Relative error is calculated as follows: $\left|\left(\log G_{B D}^{\prime}\left(\omega_{R}\right)-\log G_{t h}^{\prime}\left(\omega_{R}\right)\right) / \log \Theta\right|$, where $\Theta=$ $G_{t h}^{\prime \prime}\left(\omega_{R}=10^{2}\right) / G_{t h}^{\prime}\left(\omega_{R}=10^{-2}\right)$ is the maximal interval of complex modulus variation in the tested frequency interval

As expected, a linear evolution of the computational time $\left(2 \times\right.$ Quad-Core AMD Opteron $^{\mathrm{TM}} 2376$ $(2300 \mathrm{MHz}))$ in function of the number of trimers simulated is checked. It was corroborated that a population of $10^{4}$ systems guarantees a good compromise between computational time and rheological prediction performance. 
4.5 Non-Freely-Jointed Chains (and Non-Straight Natural Configurations)

Bending potential defined in Eq. (71) is based on the worm-like chain continuous model. It this model, the equilibrium configuration corresponds to a perfectly straight chain. This fact means that the minimum value of the internal bending energy is associated with a multi-beadrod chain whose unitary rod vectors $\mathbf{u}_{j}$ are all the same. Bending potential force acting on bead $k$ can be easily deduced as the derivative of the discrete bending potential with respect to the position of bead $k$ (Eq. (72)). Total bending force acting on bead $k$ only depends on the local chain configuration and can be interpreted as the sum of independent contributions coming from all the two-rod sub-sections containing bead $k$. Demonstration of the previous can be found elsewhere [27].

However, finite-contour-length high-aspect-ratio systems with naturally non-straight configurations at equilibrium can be found in the physical world (for example carbon nanotubes, rod-like virus, genes) and, therefore, a redefinition of the discrete bending potential taking into account a natural non-straight configuration is required to simulate the dynamic response of naturally bent structures.

In this context, a suitable redefinition of the discrete bending potential for a multi-bead-rod model with a naturally bent configuration at equilibrium can be expressed in the next way:

$\phi=-\frac{K_{b}}{a} \sum_{i=2}^{n-1} \mathbf{Z}_{i} \mathbf{u}_{i} \cdot \mathbf{u}_{i-1}=-\frac{K_{b}}{a} \sum_{i=2}^{n-1} \mathbf{u}_{i}^{\prime} \cdot \mathbf{u}_{i-1}$

where $\mathbf{Z}_{i}$ is a linear operator that counter-rotates vector $\mathbf{u}_{\mathbf{i}}$ of $\Delta \theta_{i, i-1}^{0}$ (interior angle between $\mathbf{u}_{\mathbf{i}}$ and $\mathbf{u}_{\mathbf{i}-\mathbf{1}}$ at the equilibrium configuration) and $\mathbf{u}_{i}^{\prime}$ is the rotated vector $\mathbf{Z}_{i} \mathbf{u}_{i}$. In an analogous way with the straight case, bending force coming from the potential in Eq. (100) takes the next form:

$\mathbf{F}_{k}^{\phi}=-\frac{\partial \phi}{\partial \mathbf{r}_{k}}=\frac{K_{b}}{a} \sum_{i=2}^{n-1} \frac{\partial\left(\mathbf{u}_{i}^{\prime} \cdot \mathbf{u}_{i-1}\right)}{\partial \mathbf{r}_{k}}$

Considering that the total bending force in Eq. (101) can also be interpreted as the sum of independent contributions coming from all the two-rod sub-sections containing bead $k$, as shown in [27], next algorithm is proposed for computing the total bending forces in coherence with a naturally bent configuration:

1. Decomposition of the multi-bead-rod ( $n$ beads) system into $n-2$ sub-section of two consecutive rods as shown in Fig. 24 (Note that sub-section $p$ is composed by beads $p, p+1$ and $p+2)$.

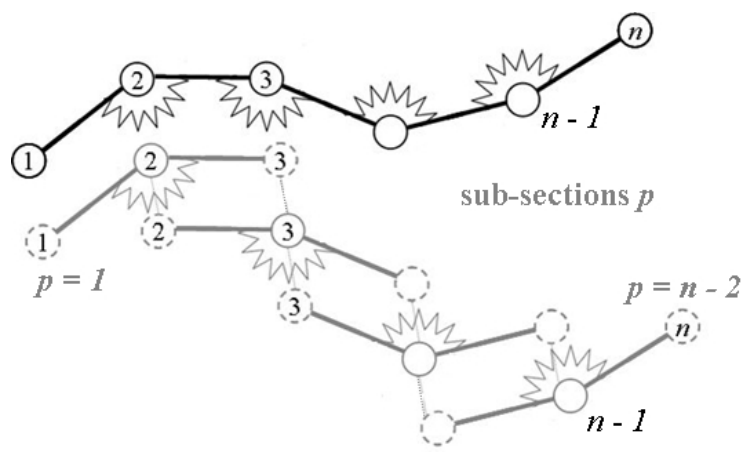

Fig. 24 Multi bead-rod model of $n$ beads (in black) decomposed in $n-2$ sub-sections $p$ constituted by two consecutive rods (in gray)

2. Calculation of the non-zero contributions to the bending forces over all beads composing each sub-section $p$ as follows:

- Bending force on bead $p$ of sub-section $p$ as:

$\mathbf{F}_{p, p}^{(\phi)}=-\frac{K_{b}}{a^{2}}\left(\boldsymbol{\delta}-\mathbf{u}_{p} \otimes \mathbf{u}_{p}\right) \cdot \mathbf{u}_{p+1}^{\prime}$

- Bending force on bead $p+2$ of sub-section $p$ as: $\mathbf{F}_{p+2, p}^{\phi}=$

$\mathbf{Z}_{p+1}^{-1} \cdot\left[\frac{K_{b}}{a^{2}}\left(\boldsymbol{\delta}-\mathbf{u}_{p+1}^{\prime} \otimes \mathbf{u}_{p+1}^{\prime}\right) \cdot \mathbf{u}_{p}\right]$

Bending force on bead $p+2$ of sub-section $p$ has been rotated back (operator $\mathbf{Z}_{p+1}^{-1}$ ) in order to correct the direction of the force, that must to be in coherence with the actual bent configuration.

- As the bending potential in each sub-section is locally independent of the adjacent sub-sections, it must to satisfy mechanical equilibrium and, hence, bending force on bead $p+1$ of sub-section $p$ writes:

$\mathbf{F}_{p+1, p}^{(\phi)}=-\mathbf{F}_{p, p}^{(\phi)}-\mathbf{F}_{p+2, p}^{(\phi)}$

3. Finally, total bending force acting on bead $k$ is calculated as follows:

$\mathbf{F}_{k}^{(\phi)}=\sum_{p=1}^{n-2} \mathbf{F}_{k, p}^{(\phi)}$

Given the previous more general definition of the bending forces for a multi-bead-rod model with any equilibrium configuration, we can deal with more scientific rigor the effects of the bending flexibility on the dynamic response of multi-bead-rod chains in dilute solution. To show this, bi-dimensional BD simulations are carried out for several non-freely-jointed (three-beads)-(two-rods) systems. In this case, hydrodynamic interaction is neglected and an isotropic friction tensor is supposed. Previous premises allowed us to implement a first-order integration scheme in coherence with the predictor-corrector algorithm; no metric potential forces are required in the formulation. Shearstress tensor contribution coming from the bead-rod 
chains is calculated with the modified Kramers Kirkwood expression using the "long-lasting" Brownian forces algorithm.

A large range of dynamic responses in linear viscoelasticity is deployed depending on the equilibrium configuration and the bending rigidity constant. To explain this, in Fig. 25 the dynamic responses of a freelyjointed system, two non-freely-jointed systems with natural straight configuration and a non-freely-jointed system with a naturally bent configuration are compared. Rod misalignment (i.e. the natural bent multi-bead-rod configuration) is generated following the next formula:

$\theta_{i}^{0}=\theta_{i-1}^{0}-\Delta \theta^{\max }+\mathrm{U}(0,1) \cdot 2 \Delta \theta^{\max }$

where $\theta_{i}^{0}$ is the equilibrium director angle of $\operatorname{rod} i$, $\Delta \theta^{\max }$ is the maximal misalignment between two consecutive rods and $\mathrm{U}(0,1)$ is a continuous uniform random distribution function defined between 0 and 1 .

In a general way, when the bending rigidity constant $K_{b}$ is equal or greater than the thermal coefficient $a k_{B} T$, the loss modulus is enhanced over the entire frequency range and the storage modulus is increased in the low frequency regime. Otherwise, if the bending rigidity constant is much lower than the thermal coefficient $\left(K_{b}<<a k_{B} T\right)$, a dynamic response similar to that one of a freely jointed system is obtained, showing just one dominant relaxation time associated with the thermal-to-flow induced motion transition.

Additionally, in Fig. 25 the apparition of new relaxation processes when the bending rigidity constant is considerably greater than the thermal coefficient (i.e. $\left.K_{b}>>a k_{B} T\right)$ is observed. For instance, the activation of a mild elasticity at intermediate frequencies is clearly noticed in the dynamic response of the nonfreely jointed (three-beads)-(two-rods) system with bending rigidity constant $K_{b}=10$. When comparing the straight configuration with the bent one, even though the loss modulus responses are similar, an interesting difference in the storage modulus is appreciated. In fact, on the one hand the activation of the mild elasticity for the system with a natural bent configuration is observed at a lower characteristic frequency than the system with a straight configuration; on the other hand, a more steeped mild elasticity is noticed for the bent system in comparison with the straight one. In front of this qualitative feature the equilibrium configuration appears a priori as an important factor affecting the dynamic response under flow of a multi bead-rod system.

In Fig. 26 the BD predictions of the complex modulus for a non-freely jointed (three-beads)-(two-rods) system with a natural bent configuration for different values of the bending rigidity constant are presented. The typical thermal-to-flow induced relaxation process

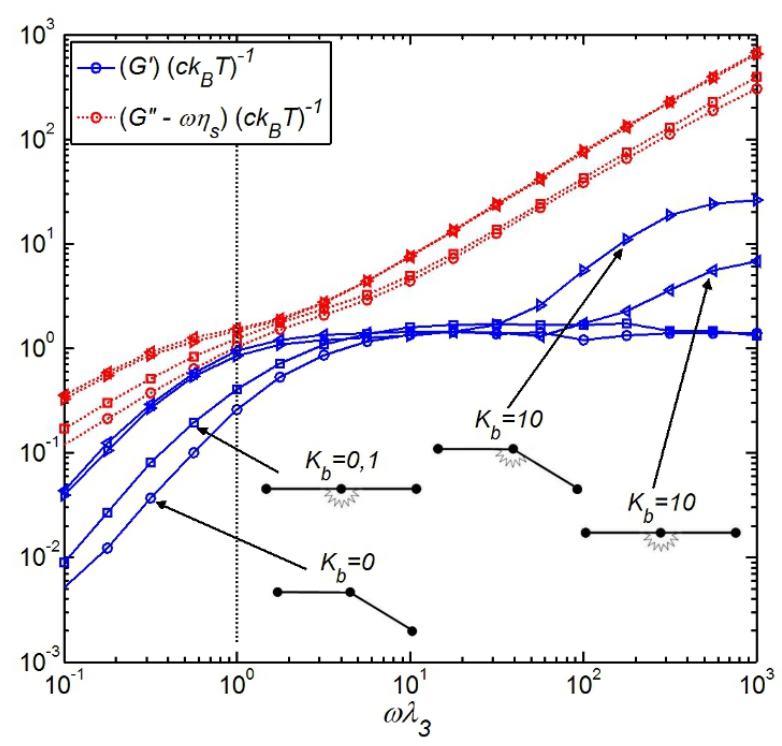

Fig. 25 BD predicted complex modulus of different (threebeads)-(two-rods) systems: freely jointed, non-freely jointed with natural straight configuration $\left(K_{b}=0.1\right.$ and $K_{b}=10$ in a consistent system of units) and non-freely-jointed with natural bent configuration $\left(K_{b}=10\right.$ in a consistent system of units and $\left.\Delta \theta^{\max }=30^{\circ}\right) . a$ and $\zeta$ are fixed to 1 and $c k_{B} T$ to 0.1 in a consistent system of units. $\lambda_{3}$ is the rotational diffusion time of a (3-beads)-(rigid-rod) system of equal length

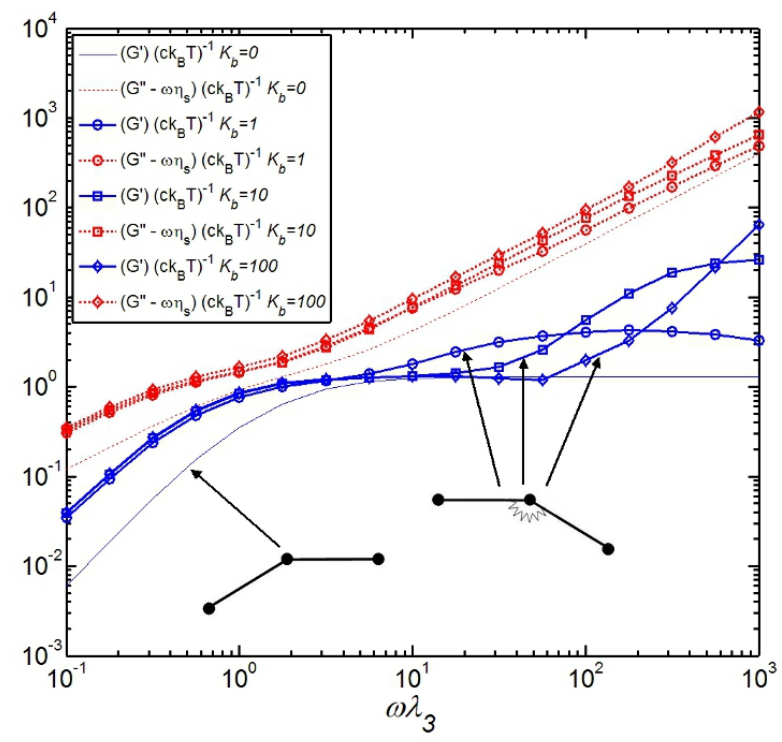

Fig. 26 BD predicted complex modulus of a non-freely jointed (three-beads)-(two-rods) system with a natural bent configuration (maximal misalignment angle $\Delta \theta^{\max }=30^{\circ}$ ) for different values of the bending rigidity constant $K_{b}$. Freely-jointed system $\left(K_{b}=0\right)$ is presented as reference. $a$ and $\zeta$ are fixed to 1 and $c k_{B} T$ to 0.1 in a consistent system of units. $\lambda_{3}$ is the rotational diffusion time of a (3-beads)-(rigidrod) system of equal length 
occurring around the rotational diffusion time $\lambda_{3}$ (as in the equivalent straight systems) is easily identifiable. Additionally, an interesting second relaxation process (as showed before) activating a mild elasticity at intermediate frequencies is again appreciated for the natural bent systems. BD simulations have showed that the characteristic frequency of this relaxation process and the magnitude of the storage modulus enhancement are function of the bending rigidity constant. The activation frequency of this mild elasticity is increased as the bending rigidity constant is increased as well. This feature is physically coherent taking into account that a higher energy input (i.e. solicitation frequency) is required for activate the first bending mode of a stiffer system. On the other hand, it is also noticed that the storage modulus enhancement and the frequency range associated to those new relaxation processes also rises as the bending rigidity increases. This qualitative feature can be physically explained considering that the stored internal energy associated with a given bending mode is directly proportional to the bending rigidity constant.

\section{Summary}

Brownian dynamics (BD) is an extensive numerical method for analyzing the dynamic behaviour of any coarsegrained structural model. In fact, BD is practically the only mathematical approach for those structural models that do not have an equivalent Fokker-Planck (FP) equation and, in addition, is an alternative approach for solving in a discrete fashion the FP equation of those models that have one. This extensive micro-to-macro approach is purely stochastic and, in consequence, relatively costly in terms of computational resources. A correct implementation of a BD simulation in computational rheology for a given structural model depends particularly on the precise formulation of an equivalent stochastic differential equation coupled with a coherent time integration algorithm and a stress tensor calculation.

This paper tackled the BD simulation of the linear viscoelastic behaviour of dilute solution of two classical models in kinetic theory: multi-bead-spring chains and multi-bead-rod chains. An algorithm for simulating a dynamic test in linear viscoelasticity (small-amplitude oscillatory flow) has been developed. The BD approach in linear viscoelasticity for the generalized bead-spring model was validated in front of the analytical solutions of the Rouse theory (freely-jointed multi-bead(Hookean-springs) chains without hydrodynamic interaction) and Zimm theory (freely-jointed multi-bead(Hookean-springs) chains with equilibrium-averaged hy- drodynamic interaction), implementing a simple Euler time-integration scheme and the Kramers expression for calculating the shear-stress tensor.

On the other hand, the BD simulation in linear viscoelasticity for the generalized bead-rod model was validated with respect to the exact numerical solution for the freely-jointed (three-beads)-(two-rods) chain given by Hassager, implementing an explicit time-integration scheme and the Kramers-Kirkwood expression coupled with a "long-lasting" Brownian forces algorithm for calculating the shear-stress tensor. Moreover, it was shown that a bi-dimensional BD modelling allows to correctly approach the dynamic behaviour of classical 3D beadrod-spring chain models.

The dynamic response of non-freely-jointed multibead-rod chains with naturally straight and non-straight configuration was also simulated. In terms of numerical implementation of the naturally non-straight configuration, a mechanically coherent re-definition of the discretized version of the bending potential for naturally straight semi-flexible filaments was necessary in order to tackle properly the cases of bent configurations. An algorithm by decomposing the multi-bead-rod chain in (three-beads)-(two-rods) sub-sections was developed with the aim of calculating the forces coming from the internal bending potential.

From the BD simulation results, non-negligible differences in the rheological responses were found between the naturally straight and the naturally bent models. BD simulation of bead-spring models was demonstrated to be more straightforward than the simulation of bead-rod chains. The presence of physical constraints imposes several restrictions in terms of Brownian forces generation, time-integration algorithms and stress tensor calculation that are reflected directly on the implementation of the BD simulation.

\section{References}

1. Kröger M. Models for Polymeric and Anisotropic Liquids. Springer, Berlin Heidelberg, 2005.

2. Langevin P. Sur la théorie du mouvement brownien. Comptes Rendus, 146:530, 1908.

3. Ottinger HC. Stochastic Processes in Polymeric Fluids. Springer, Berlin Heidelberg, 1996.

4. Ermak DL and McCammon JA. Brownian dynamics with hydrodynamic interactions. J Chem Phys, 69(4):13521360, 1978.

5. Pear MR and Weiner JH. Brownian dynamics study of a polymer chain of linked rigid bodies. ii. results for longer chains. J Chem Phys, 72(7):3939-3947, 1980.

6. Hatzikiriakos SG and Vlassopoulos D. Brownian dynamics simulations of shear-thickening in dilute polymer solutions. Rheo Acta, 35(3):274-287, 1996.

7. Agarwal US, Bhargava R, and Mashelkar RA. Brownian dynamics simulation of a polymer molecule in solution 
under elongational flow. J Chem Phys, 108(4):1610-1617, 1998.

8. Cass MJ, Heyes DM, and English RJ. Brownian dynamics simulations of associating diblock copolymers. Langmuir, 23(12):6576-6587, 2007.

9. Jin S and Collins LR. Dynamics of dissolved polymer chains in isotropic turbulence. New J Phys, 9, 2007.

10. Venkataramani V, Sureshkumar R, and Khomami B. Coarse-grained modeling of macromolecular solutions using a configuration-based approach. $J$ Rheol, 52(5):1143$1177,2008$.

11. Dwyer JD and Bloomfield VA. Brownian dynamics simulations of probe and self-diffusion in concentrated protein and dna solutions. Biophys J, 65(5):1810-1816, 1993.

12. Merlitz H, Rippe K, Kienin KV, and Langowski J. Looping dynamics of linear dna molecules and the effect of dna curvature: A study by brownian dynamics simulation. Biophys J, 74(2I):773-779, 1998.

13. García de la Torre J, Pérez Sánchez HE, Ortega A, Hernández JG, Fernandes MX, and Díaz FG. Calculation of the solution properties of flexible macromolecules: Methods and applications. Eur Biophys J, 32(5):477-486, 2003.

14. Kenward M and Dorfman KD. Coarse-grained brownian dynamics simulations of the 10-23 dnazyme. Biophys $J$, 97(10):2785-2793, 2009.

15. Ansell GC and Dickinson E. Brownian dynamics simulation of the fragmentation of a large colloidal floc in simple shear flow. J Colloid Interf Sci, 110(1):73-81, 1986.

16. Xue $\mathrm{W}$ and Grest GS. Brownian dynamics simulations for interacting colloids in the presence of a shear flow. Phys Rev A, 40(3):1709-1712, 1989.

17. Heyes DM and Branka AC. Brownian dynamics simulations of self-diffusion and shear viscosity of near-hardsphere colloids. Phys Rev E, 50(3):2377-2380, 1994.

18. Bijsterbosch BH, Bos MTA, Dickinson E, Van Opheusden JHJ, and Walstra P. Brownian dynamics simulation of particle gel formation: From argon to yoghurt. Faraday Discuss, 101:51-64, 1995.

19. Tokuyama M. Effective diffusion model on brownian dynamics of hard-sphere colloidal suspensions. Physica A, 265(3):333-340, 1999.

20. Miyahara M, Watanabe S, and Higashitani K. Modeling adsorption and order formation by colloidal particles on a solid surface: A brownian dynamics study. Chem Eng Sci, 61(7):2142-2149, 2006.

21. Schmidt M, Patrick Royall C, Van Blaaderen A, and Dzubiella J. Non-equilibrium sedimentation of colloids: Confocal microscopy and brownian dynamics simulations. $J$ Phys-Condens Mat, 20(49), 2008.

22. Mori N, Fujioka H, Semura R, and Nakamura K. Brownian dynamics simulations for suspension of ellipsoids in liquid crystalline phase under simple shear flows. Rheol Acta, 42(1):102-109, 2003.

23. Siettos CI, Graham MD, and Kevrekidis IG. Coarse brownian dynamics for nematic liquid crystals: Bifurcation, projective integration, and control via stochastic simulation. J Chem Phys, 118(22):10149-10156, 2003.

24. Song YS and Youn JR. Modeling of rheological behavior of nanocomposites by brownian dynamics simulation. Korea-Aust Rheol J, 16(4):201-212, 2004.

25. Mendes MJ, Schmidt HK, and Pasquali M. Brownian dynamics simulations of single-wall carbon nanotube separation by type using dielectrophoresis. J Phys Chem B, 112(25):7467-7477, 2008.

26. Fakhri N, Tsyboulski DA, Cognet L, Bruce Weisman R, and Pasquali M. Diameter-dependent bending dynamics of single-walled carbon nanotubes in liquids. P Natl Acad Sci USA, 106(34):14219-14223, 2009.

27. Cruz C, Illoul L, Chinesta F, and Régnier G. Effects of a bent structure on the linear viscoelastic response of diluted carbon nanotube suspensions. Rheologica Acta, 49(11):1141-1155, 2010.

28. Bird RB, Curtiss CF, Amstrong RC, and Hassager O. Dynamics of polymer liquids, volume 2 Kinetic Theory. Wyley-Interscience, second edition, 1987.

29. Flory PJ. Statistical mechanics of chain molecules. Wiley-Interscience, New York, 1969.

30. Kirkwood JG. Macromolecules, J. G. Kirkwood Collected Works. Gordon and Breach, New York, 1967.

31. Larson RG. The Structure and Rheology of Complex Fluids. Oxford University Press, 1999.

32. Nayac R. Molecular simulation of liquid crystal polymer flow: a wavelet- finite element analysis. PhD thesis, MIT, Cambridge, MA, USA, 1998.

33. Lozinski A and Chauviere C. A fast solver for fokkerplanck equation applied to viscoelastic flows calculations: 2d fene model. J Comput Phys, 189(2):607-625, 2003.

34. Lozinski A, Chauviere C, Fang J, and Owens RG. Fokkerplanck simulations of fast flows of melts and concentrated polymer solutions in complex geometries. J Rheol, 47(2):535-561, 2003.

35. Chauviere C, Fang J, Lozinski A, and Owens RG. On the numerical simulation of flows of polymer solutions using high-order methods based on the fokker-planck equation. Int J Mod Phys B, 17(1-2):9-14, 2003.

36. Suen JK, Nayak R, Armstrong RC, and Brown RA. A wavelet-galerkin method for simulating the doi model with orientation-dependent rotational diffusivity. J NonNewton Fluid, 114(2-3):197-228, 2003.

37. Chauviere C and Lozinski A. Simulation of dilute polymer solutions using a fokker-planck equation. Comput Fluids, 33(5-6):687-696, 2004.

38. Ammar A, Ryckelynck D, Chinesta F, and Keunings R. On the reduction of kinetic theory models related to finitely extensible dumbbells. J Non-Newton Fluid, 134(1-3 SPEC. ISS.):136-147, 2006.

39. Ammar A, Mokdad B, Chinesta F, and Keunings R. A new family of solvers for some classes of multidimensional partial differential equations encountered in kinetic theory modeling of complex fluids. J Non-Newton Fluid, 139(3):153-176, 2006.

40. F. Chinesta, A. Ammar, A. Falco, and M. Laso. On the reduction of stochastic kinetic theory models of complex fluids. Model Simul Mater Sc, 15(6):639-652, 2007.

41. B. Mokdad, E. Pruliere, A. Ammar, and F. Chinesta. On the simulation of kinetic theory models of complex fluids using the fokker-planck approach. Appl Rheol, 17(2):26494-1-26494-14, 2007.

42. A. Ammar, F. Chinesta, and P. Joyot. The nanometric and micrometric scales of the structure and mechanics of materials revisited: An introduction to the challenges of fully deterministic numerical descriptions. Int J Multiscale Com, 6(3):191-213, 2008.

43. A. Ammar, E. Pruliere, F. Chinesta, and M. Laso. Reduced numerical modeling of flows involving liquidcrystalline polymers. J Non-Newton Fluid, 160(2-3):140156, 2009.

44. E. Pruliere, A. Ammar, N. El Kissi, and F. Chinesta. Recirculating flows involving short fiber suspensions: $\mathrm{Nu}-$ merical difficulties and efficient advanced micro-macro solvers. Arch Comput Method E, 16(1):1-30, 2009.

45. G. M. Leonenko and T. N. Phillips. On the solution of the fokker-planck equation using a high-order reduced basis 
approximation. Comput Method Appl M, 199(1-4):158168,2009

46. A. Ammar, M. Normandin, F. Daim, D. Gonzalez, E. Cueto, and F. Chinesta. Non-incremental strategies based on separated representations: Applications in computational rheology. Commun Math Sci, 8(3):671-695, 2010.

47. A. Ammar, M. Normandin, and F. Chinesta. Solving parametric complex fluids models in rheometric flows. $J$ Non-Newton Fluid, 165(23-24):1588-1601, 2010.

48. B. Mokdad, A. Ammar, M. Normandin, F. Chinesta, and J. R. Clermont. A fully deterministic micro-macro simulation of complex flows involving reversible network fluid models. Math Comput Simulat, 80(9):1936-1961, 2010.

49. F. Chinesta, A. Ammar, A. Leygue, and R. Keunings. An overview of the proper generalized decomposition with applications in computational rheology. J Non-Newton Fluid, 166(11):578-592, 2011.

50. Laso M and Öttinger HC. Calculation of viscoelastic flow using molecular models: the connffessit approach. $J$ NonNewton Fluid, 47(C):1-20, 1993.

51. Feigl K, Laso M, and Öttinger HC. Connffessit approach for solving a two-dimensional viscoelastic fluid problem. Macromolecules, 28(9):3261-3274, 1995.

52. Hua CC and Schieber JD. Application of kinetic theory models in spatiotemporal flows for polymer solutions, liquid crystals and polymer melts using the connffessit approach. Chem Eng Sci, 51(9):1473-1485, 1996.

53. Laso M, Picasso M, and Öttinger HC. 2-D timedependent viscoelastic flow calculations using CONNFFESSIT. AICHE J, 43(4):877-892, 1997.

54. Huang ZP, Xu JW, Ren ZF, Wang JH, Siegal MP, and Provencio PN. Growth of highly oriented carbon nanotubes by plasma-enhanced hot filament chemical vapor deposition. Appl Phys Lett, 73(26):3845-3847, 1998.

55. Cormenzana J, Ledda A, Laso M, and Debbaut B. Calculation of free surface flows using connffessit. J Rheol, 45(1):237-258, 2001.

56. Keunings R. Micro-Macro Methods for the Multiscale Simulation of Viscoelastic Flow using Molecular Models of Kinetic Theory, pages 67-98. British Society of Rheology, 2004.

57. Rouse PE. A theory of the linear viscoelastic properties of dilute solutions of coiling polymers. J Chem Phys, 21(7):1272-1280, 1953.

58. Zimm B. Dynamics of polymer molecules in dilute solution: viscoelasticity, flow birefringence and dielectric loss. $J$ Chem Phys, 24(2):269-278, 1956.

59. Warner HR. Kinetic theory and rheology of dilute suspensions of finitly extendible dumbbells. Ind Eng Chem Fundamen, 11(3), 1972.

60. Treloar LRG. The physics of rubber elasticity. Oxford University Press, London, 1975.

61. Marko JF and Siggia ED. Stretching dna. Macromolecules, 28(26), 1995.

62. Higham DJ. An algorithmic introduction to numerical simulation of stochastic differential equations. SIAM Review, 43(3):525-546, 2001.

63. Somasi M, Khomami B, Woo NJ, Hur JS, and Shaqfeh ESG. Brownian dynamics simulations of bead-rod and bead-spring chains: Numerical algorithms and coarsegraining issues. J Non-Newton Fluid, 108(1-3):227-255, 2002.

64. Atkinson J, Goh CJ, and Phan-Thien N. Bead-spring models for an adsorbed polymer molecule in a shear flow. J Chem Phys, 80(12):6305-6317, 1983.
65. Fetsko SW and Cummings PT. Brownian dynamics simulation of bead-spring chain models for dilute polymer solutions in elongational flow. J Rheol, 39(2):285-299, 1995.

66. Fixman M. Implicit algorithm for brownian dynamics of polymers. Macromolecules, 19(4):1195-1204, 1986.

67. Kramers HA. The behavior of macromolecules in inhomogeneous flow. J Chem Phys, 14(7):415-424, 1946.

68. Bird RB, Warner HR, and Evans DC. Advances in Polymer Science, volume 8. Springer, Berlin Heidelberg, 1971.

69. Macosko CW. Rheology. Principles, measurements and applications. VCH, 1994.

70. Vattulainen I, Ala-Nissila T, and Kankaala K. Physical tests for random numbers in simulations. Phys Rev Lett, 73(19):2513-2516, 1994.

71. Kirkwood JG and Riseman J. The intrinsic viscosities and diffusion constants of flexible macromolecules in solution. J Chem Phys, 16(6):465-573, 1948.

72. Thurston GB and Peterlin A. Influence of finite number of chain segments, hydrodynamic interaction, and internal viscosity on intrinsic birefringence and viscosity of polymer solutions in an oscillating laminar flow field. $J$ Chem Phys, 46(12):4881-4885, 1967.

73. Osaki K, Schrag JL, and Ferry JD. Infinite-dilution viscoelastic properties of poly( $\alpha$-methylstyrene). applications of zimm theory with exact eigenvalues. Macromolecules, 5(2):144-147, 1972.

74. Fixman M. Construction of langevin forces in the simulation of ydrodynamic interaction. Macromolecules, 19(4):1204-1207, 1986.

75. Ferry JD. Viscoelastic properties of polymers. John Wiley \& Sons, New Jersey, second edition, 1970.

76. Liu TW. Flexible polymer chain dynamics and rheological properties in steady flows. J Chem Phys, 90(10):58265842,1989

77. Hassager O. Kinetic theory and rheology of bead-rod models for macromolecular solutions. J Chem Phys, 60(5):2111-2124, 1974.

78. Fan XJ and Liu TW. Equilibrium and steady-state flow properties of a suspension of freely rotating chains. $J$ Non-Newton Fluid, 19(3):303-321, 1986.

79. Fixman M. Simulation of polymer dynamics. ii. relaxation rates and dynamic viscosity. $J$ Chem Phys, 69(4):1538-1545, 1978

80. Gottlieb M and Bird RB. A molecular dynamics calculation to confirm the incorrectness of the random-walk distribution for describing the kramers freely jointed beadrod chain. J Chem Phys, 65(6):2467-2468, 1976.

81. Pear MR and Weiner JH. Brownian dynamics study of a polymer chain of linked rigid bodies. J Chem Phys, 71(1):212-224, 1979.

82. Fixman M. Simulation of polymer dynamics. i. general theory. J Chem Phys, 69(4):1527-1537, 1978.

83. Helfand E. Flexible vs rigid constraints in statistical mechanics. J Chem Phys, 71(12):5000-5007, 1979.

84. Hinch EJ. Brownian motion with stiff bonds and rigid constraints. J Fluid Mech, 271:219-234, 1994.

85. Grassia PS, Hinch EJ, and Nitsche LC. Computer simulations of brownian motion of complex systems. J Fluid Mech, 282:373-403, 1995.

86. Grassia P and Hinch EJ. Computer simulations of polymer chain relaxation via brownian motion. J Fluid Mech, 308:255-288, 1996.

87. Pasquali $\mathrm{M}$ and Morse DC. An efficient algorithm for metric correction forces in simulations of linear polymers with constrained bond lengths. J Chem Phys, 116(5):1834-1838, 2002. 
88. Shankar V, Pasquali M, and Morse DC. Theory of linear viscoelasticity of semiflexible rods in dilute solution. $J$ Rheol, 46(5):1111-1154, 2002.

89. Montesi A, Morse DC, and Pasquali M. Brownian dynamics algorithm for bead-rod semiflexible chain with anisotropic friction. J Chem Phys, 122(084903), 2005.

90. Morse DC. Viscoelasticity of concentrated isotropic solutions of semiflexible polymers. 1 . model and stress tensor. Macromolecules, 31(20):7030-7043, 1998.

91. Allison SA and McCammon JA. Multistep brownian dynamics: application to short wormlike chains. Biopolymers, 23(2):363-375, 1984.

92. Ermak DL and McCammon JA. Brownian dynamics with hydrodynamic interactions. J Chem Phys, 69(4):13521360, 1978.

93. Doyle PS, Shaqfeh ESG, and Gast AP. Dynamic simulation of freely draining flexible polymers in steady linear flows. J Fluid Mech, 334:251-291, 1997.

94. Morse DC. Theory of constrained brownian motion. Adv Chem Phys, 128:65-189, 2004.

95. Fixman M and Kovac J. Dynamics of stiff chains. ii. freely jointed chain. J Chem Phys, pages 4950-4955, 1974. 